\title{
INCENTIVE CONSTRAINED RISK SHARING, SEGMENTATION, AND ASSET PRICING
}

\author{
Bruno Biais \\ Johan Hombert \\ Pierre-Olivier Weill \\ Working Paper 23986 \\ http://www.nber.org/papers/w23986
}

\author{
NATIONAL BUREAU OF ECONOMIC RESEARCH \\ 1050 Massachusetts Avenue \\ Cambridge, MA 02138 \\ November 2017, Revised November 2020
}

We'd like to thank, for fruitful comments and suggestions, the editor Mikhail Golosov and the four referees as well as Andrea Attar, Andy Atkeson, Saki Bigio, Jason Donaldson, James Dow, Ana Fostel, Zhiguo He, Alfred Galichon, Valentin Haddad, Thomas Mariotti, Simon Mongey, Ed Nosal, Adriano Rampini, Bruno Sultanum, Jean Tirole, Aleh Tsyvinski, Venky Venkateswaran, Bill Zame, Fei Zhou, and Diego Zúñiga, as well as seminar participants at UCLA, the AQR conference at the LBS, the Banque de France Workshop on Liquidity and Markets, the Finance Theory Group conference at the LSE, the Gerzensee Study Center, MIT, Washington University in St. Louis Olin Business School, the LAEF conference on Information in Financial Markets, EIEF, University of Geneva, University of Virginia, Princeton University, Penn State, Cornell, University of British Columbia, Simon Fraser University, Federal Reserve Bank of New York, Imperial College, UCL, the 8th Summer Macro-Finance Workshop in Sciences Po, the Federal Reserve Board, the Federal Reserve Bank of Minneapolis, the Banque de France, the University of California in Santa Cruz, Carnegie Mellon University, the University of Colorado, Yale University, the Hong Kong Baptist University, and UCI. Diego Zúñiga provided expert research assistance. We thank the Bank of France for financial support. The views expressed herein are those of the authors and do not necessarily reflect the views of the National Bureau of Economic Research.

At least one co-author has disclosed additional relationships of potential relevance for this research. Further information is available online at http://www.nber.org/papers/w23986.ack

NBER working papers are circulated for discussion and comment purposes. They have not been peer-reviewed or been subject to the review by the NBER Board of Directors that accompanies official NBER publications.

(C) 2017 by Bruno Biais, Johan Hombert, and Pierre-Olivier Weill. All rights reserved. Short sections of text, not to exceed two paragraphs, may be quoted without explicit permission provided that full credit, including $\odot$ ) notice, is given to the source. 
Incentive Constrained Risk Sharing, Segmentation, and Asset Pricing

Bruno Biais, Johan Hombert, and Pierre-Olivier Weill

NBER Working Paper No. 23986

November 2017, Revised November 2020

JEL No. D5,G12

\begin{abstract}
$\underline{\text { ABSTRACT }}$
Incentive problems make securities' payoffs imperfectly pledgeable, limiting agents' ability to issue liabilities. We analyze the equilibrium consequences of such endogenous incompleteness in a dynamic exchange economy. Because markets are endogenously incomplete, agents have different intertemporal marginal rates of substitution, so that they value assets differently. Consequently, agents hold different portfolios. This leads to endogenous markets segmentation, which we characterize with Optimal Trans-port methods. Moreover, there is a basis going always in the same direction: the price of a security is lower than that of replicating portfolios of long positions. Finally, equilibrium expected returns are concave in factor loadings.
\end{abstract}

Bruno Biais

Toulouse School of Economics (CNRS, IDEI)

Manufacture des Tabacs

21, allée de Brienne

31000 TOULOUSE

FRANCE

bruno.biais@univ-tlse1.fr

Johan Hombert

HEC Paris

1 rue de la Liberation

78351 Jouy en Josas

France

hombert@hec.fr
Pierre-Olivier Weill

Department of Economics

University of California, Los Angeles

Bunche Hall 8283

Los Angeles, CA 90095

and NBER

poweill@econ.ucla.edu 


\section{Introduction}

One of the key functions of financial markets is to enable agents to share risk. For example, relatively risk tolerant market participants can sell puts or Credit Default Swaps to more risk averse agents, or agents with larger risk exposure. We study risk sharing in general equilibrium in a dynamic exchange economy. At each period agents receive heterogeneous endowments of consumption good (labor income), as well as the output or "dividends" of the assets, or "trees", they hold. At each period there is also a complete set of Arrow securities, spanning endowments and dividends, in zero net supply.

Agents' liabilities, modeled as sales of Arrow securities, are backed by the assets they can pledge as collateral. Should these participants default, however, seizing their collateral could prove difficult and costly. This has been documented for variety of collateral assets, for example residential homes backing mortgages (Campbell, Giglio and Pathak, 2011), productive assets backing firms' liabilities (Andrade and Kaplan, 1998), and traded assets backing financial firms liabilities (Fleming and Sarkar, 2014).

That seizing collateral is difficult and costly creates scope for opportunistic debtors' behaviour. In Kiyotaki and Moore (1997), strategic debtors use the threat of costly bankruptcy to negotiate debt down to liquidation values. We consider a similar mechanism. Suppose an agent issued Arrow securities, promising to pay a given amount should a given state occur. When that state realizes, the agent can threaten to default on her promise. Suppose that, in case of default, the buyer of the security can only seize a fraction $1-\theta$ of the assets of the defaulting agent, while the fraction $\theta$ is deadweight bankruptcy cost. In this context, if the agent can make a take-it-or-leave-it offer to the Arrow security buyer, she can renegotiate her liability to a fraction $1-\theta$ of the value of her asset holdings. We refer to this value as the pledgeable income of the agent, and to the constraint that the agent cannot promise more than her pledgeable income as the incentive constraint. Imperfect pledgeability implies over-collateralization and limits agents' ability to sell Arrow securities, which generates endogenous market incompleteness. The goal of this paper is to study the consequences of incentive constraints and imperfect collateral pledgeability for risk sharing, portfolio choice, and asset and derivative pricing.

Because, in addition to classical budget constraints, agents face incentive constraints in which prices 
enter, standard equilibrium existence proofs based on Welfare Theorems do not apply in our framework. In particular, the approach pioneered by Negishi (1960) cannot be used. Yet, we prove equilibrium existence, extending the price-player proof of Arrow and Debreu (1954) to our setting.

In a frictionless complete market equilibrium, intertemporal marginal rates of substitution are equalized across agents. This yields a valuation operator (or pricing kernel), common to all agents, which prices all securities. In contrast, when incentive constraints limit risk sharing, agents have different intertemporal marginal rates of substitution and thus have different private valuations for imperfectly pledgeable assets. In equilibrium, each tree is held by the agent who values it most. Because agents value trees differently, they hold strictly different portfolios, i.e., there is endogenous segmentation. Intuitively, agents choose tree portfolios that, in combination with their labor income, come close to replicate their desired consumption profile. Then, to further approach their desired consumption, agents either buy Arrow securities, or they use their tree portfolios as collateral to sell Arrow securities. Theoretically, we show that the equilibrium allocation of trees to agents solves the classical Optimal Transport problem of drawing power diagrams (Galichon, 2016, Chapter 5). Empirically, equilibrium segmentation is in line with evidence from household finance. For example, Catherine, Sodini and Zhang (2020) find that "workers facing higher left-tail income risk when equity markets perform poorly are less likely to participate in the stock market."

In our framework as in previous models of endogenously incomplete markets (notably Alvarez and Jermann, 2000), only agents whose incentive constraint does not bind in a given state have intertemporal marginal rate of substitution, i.e., private valuations, equal to the Arrow security price of that state. And it is those agents who in equilibrium buy these Arrow securities. In contrast, the other agents' intertemporal marginal rates of substitution for that state are lower than the Arrow security price. They sell Arrow securities until their incentive constraint binds.

Therefore, as soon as an agent's incentive constraint binds in at least one state, this agent's private valuation for a tree paying off in that state is lower than the price of a replicating portfolio of Arrow securities. When this is true for all agents, the tree is priced below its replicating portfolio, i.e., there is a basis. More generally, any asset is priced below any portfolio of long positions in assets or securities that replicates its payoff. Such deviations from the Law of One Price are equilibrium phenomena, which cannot 
be arbitraged. To engage in arbitrage, one would have to sell the expensive leg of the arbitrage, i.e., sell Arrow securities. Such sales, however, would violate incentive constraints.

A tree can be viewed as a bundle of imperfectly pledgeable payoffs in different states. It is priced below any replicating portfolio of long positions in assets and securities because each asset in the replicating portfolio can be held by the agent who values it most, whereas the tree must be held by a unique agent. The inequality is strict when there is no agent who has the highest private valuation for all the assets in the replicating portfolio.

For example, the payoff of a convertible bond is identical to the payoff of a portfolio combining a straight bond and a call option. In line with empirical evidence (Mitchell and Pulvino, 2012), our model implies that in equilibrium convertible bonds can be priced strictly below the replicating portfolio of straight bond and call. In practice, to take advantage of that arbitrage opportunity, market participants such as hedge funds buy convertibles and issue straight bonds and calls. Such arbitrage is constrained, however, both in practice as in our model, by the limited ability of hedge funds to issue the replicating securities.

The observation that trees are less valuable than replicating portfolios suggests that equilibrium outcomes are not invariant to changes in the tree supply, holding aggregate tree dividend and everything else the same. In particular, breaking up trees into replicating portfolios changes the tree supply in a way that relaxes incentive constraints and improves risk sharing. In contrast, when trees are fully pledgeable, the manner in which aggregate dividends are split across trees is irrelevant.

The basis between trees and replicating portfolios has a prediction for the cross-section of expected returns. Project the returns of the trees on a set of factors. If the residual of this projection are orthogonal to the agents' private valuations (which is the case, in particular, when the factors are themselves the agents' private valuation), then expected returns are concave in factor loadings. Our theoretical result that equilibrium returns are concave in factor loadings, i.e., betas, is consistent with the empirical finding that the security market line is concave (Frazzini and Pedersen, 2014; Hong and Sraer, 2016). 


\section{Related literature}

Our analysis of dynamic general equilibrium and endogenous incompleteness is in line with the seminal analyses of Kehoe and Levine (1993), Alvarez and Jermann (2000), Chien and Lustig (2009) and Gottardi and Kubler (2015). ${ }^{1}$ The main difference between our model and theirs is that we consider assets that are, at the same time, imperfectly pledgeable and tradeable. Thus, we import in a limited enforcement asset pricing model similar to Chien and Lustig (2009), the assumption that assets are imperfectly pledgeable, as in Rampini and Viswanathan (2010). The main difference between our analysis and Rampini and Viswanathan (2010) is that they analyze a production economy with investment, but take asset prices as exogenously given, while we consider an exchange economy, but endogenize prices. The main difference between our results and Kehoe and Levine (1993), Alvarez and Jermann (2000), Chien and Lustig (2009) and Gottardi and Kubler (2015) is that we obtain equilibrium deviations from the Law of One Price and endogenous segmentation.

Our result that market imperfections lead to deviations from the Law of One Price is in line with Hindy and Huang (1995), Gromb and Vayanos (2002, 2017), Gârleanu and Pedersen (2011). One difference is that we provide a micro-foundation for financial constraints in terms of imperfect collateral pledgeability. This leads to our new result that markets are endogenously segmented. In Gromb and Vayanos (2002) in contrast, segmentation is exogenous. Also, while Gârleanu and Pedersen (2011) study bases among assets and securities with different exogenous margin constraints, in our model all assets and securities have identical pledgeability, yet bases for different assets are endogenously different.

Geanakoplos and Zame (2014), Fostel and Geanakoplos (2008), Brumm, Grill, Kubler and Schmedders (2015), Geerolf (2015), and Lenel (2017) also analyze general equilibrium under collateral constraints. In that literature, each financial promise must be backed by its own collateral, which gives rise to overcollateralization as shown by Araújo, Kubler and Schommer (2010). ${ }^{2}$ In our framework, by contrast, the constraint applies to the portfolio of assets and Arrow securities of an agent, in line with the practice of portfolio margining. ${ }^{3}$ Yet, imperfect pledgeability generates over-collateralization.

\footnotetext{
${ }^{1}$ Lustig and Van Nieuwerburgh (2010) analyze empirical implications of this framework.

${ }^{2}$ For example, the same asset generating strictly positive output in two states, cannot be used to collateralize the issuance of two Arrow securities, promising payments in these two states.

${ }^{3}$ For example, on http://www.cboe.com/products/portfolio-margining-rules, one can read: "The portfolio margining rules have the effect of aligning the amount of margin money ... to the risk of the portfolio as a whole, calculated through
} 
In our model pledgeable payoffs are discounted less than non-pledgeable ones. This is in line with the collateral premium analyzed by Geanakoplos and Zame (2014), Fostel and Geanakoplos (2008), and the liquidity premium derived by the new monetarist literature (see, for example, Lagos, 2010; Li, Rocheteau and Weill, 2012; Lester, Postlewaite and Wright, 2012; Venkateswaran and Wright, 2013; Jacquet, 2015). Moreover, while the pricing of pledgeable payoffs is linear and based on a single stochastic discount factor, the pricing of non-pledgeable payoffs is non-linear and convex, based on multiple stochastic discount factors. This implies that equally pledgeable payoffs are priced differently depending on their state-contingent structure, leading to bases between assets and replicating portfolios, and to concave factor pricing.

Methodologically, our paper shows that the incentive-constrained allocation of assets across agents can be characterized with techniques from Optimal Transport theory. This means that the problem of pricing and allocating assets (bundles of risk) to heterogenous agents is economically similar to that of compensating and assigning workers (bundles of skills) to heterogenous firms. See Rosen (1983), Heckman and Scheinkman (1987) and, more recently, Edmond and Mongey (2020). We contribute to the analysis of this problem by considering state-contingent borrowing, in effect an imperfect technology to unbundle risks, and by making the assignment problem dynamic. Another difference is that, in our setting, Welfare Theorems do not hold, so existence cannot be established via optimization.

The remainder of this paper has two parts: Section 2 describes the model and Section 3 analyzes the equilibrium. The main proofs are in the appendix, and secondary ones are in the online appendix.

\section{Model}

\subsection{Assets and agents}

There is a finite number of time periods $t \in\{0,1, \ldots, T\}$. Every period a new state is drawn from some finite set $S$. We let $s_{t} \in S$ denote the state in period $t, s^{t}=\left(s^{t-1}, s_{t}\right)$ the history of states until $t$, and $S^{t}$ the set of time- $t$ histories starting from $s_{0}$. The probability of history $s^{t}$, conditional on $s_{0}$, is denoted by $\pi_{t}\left(s^{t}\right)$ and is assumed to be strictly positive. A node in the event tree is a pair $\left(t, s^{t}\right)$ of time $t \leq T$ and history $s^{t} \in S^{t}$.

simulating market moves up and down, and accounting for offsets between and among all products held..." 


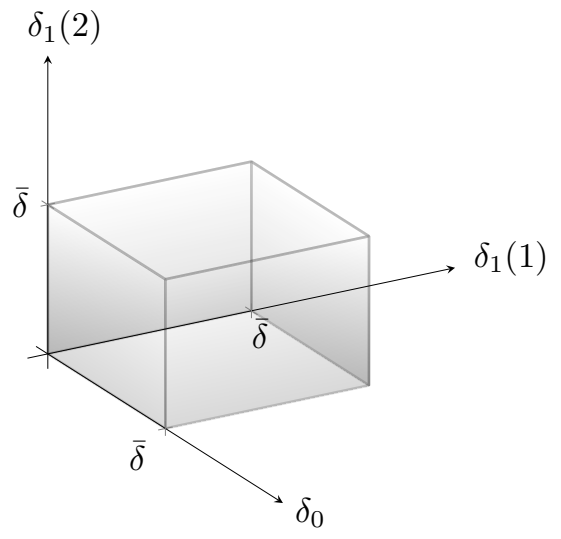

Figure 1: The set $\Delta$ when there are two periods, $t \in\{0,1\}$, and two states, $S=\{1,2\}$.

At every node $\left(t, s^{t}\right), t<T$, there is a complete set of Arrow securities in zero net supply. In addition to Arrow securities, there are trees in positive supply. A tree is defined by its dividend stream $\delta \equiv\left\{\delta_{t}\left(s^{t}\right)\right.$ : $\left.t \geq 0, s^{t} \in S^{t}\right\}$, i.e., the collection of its dividend payouts for all nodes. We do not impose any restriction on the set $\Delta$ of dividend streams except that $\delta_{t}\left(s^{t}\right) \in[0, \bar{\delta}] .{ }^{4}$ Figure 1 illustrates. For example, the set $\Delta$ can contain short-lived trees with payoffs identical to Arrow securities, long-lived trees, bonds of arbitrary maturity, and so on.

We represent the supply of trees by some positive and finite measure $\bar{N}$ over the set $\Delta$, endowed with its Borel $\sigma$-algebra. A special case is the standard model with a finite number of trees in positive supply. But our results apply equally to arbitrary supplies over $\Delta$, defined by continuous measures, discrete measures, or mixtures of both. This added generality serves several purposes. First, it clarifies the analysis of market segmentation, by providing simple geometrical representations for the equilibrium allocation trees, and establishing connections with classical results in Optimal Transport theory. Second, it demonstrates that our results are not driven by some form of tree-market incompleteness.

To facilitate the proof of equilibrium existence, we assume that the distribution of tree supplies is such that the aggregate dividend is strictly positive at all nodes, that is:

$$
\int \delta_{t}\left(s^{t}\right) d \bar{N}(\delta)>0
$$

\footnotetext{
${ }^{4}$ The upper bound $\bar{\delta}$ is arbitrary and can be viewed as a normalization, since agents can always increase the dividend payout of a tree proportionally at all nodes by scaling up their holdings. Technically, the upper bound facilitates the analysis because it makes both the set of trees, $\Delta$, and the set of positive measure over $\Delta$, compact in appropriate topologies (for the latter, see Chapter 15 in Aliprantis and Border, 2006).
} 
for all $\left(t, s^{t}\right)$, where the integral is taken over $\Delta$ and $d \bar{N}(\delta)$ is the supply of trees with dividend streams $\delta .^{5}$

On the other side of the market there is a finite number of agent's types indexed by $i$, with a measure one of each, who order consumption plans $c_{i} \equiv\left\{c_{i t}\left(s^{t}\right), 0 \leq t \leq T, s^{t} \in S^{t}\right\}$ according to the intertemporal utility:

$$
\sum_{\left(t, s^{t}\right)} \beta^{t} \pi_{t}\left(s^{t}\right) u_{i}\left(c_{i t}\left(s^{t}\right)\right)
$$

where $u_{i}(c)$ is strictly increasing, concave, continuous and continuously differentiable over $c>0$. We also assume continuity at $c=0$ unless $u_{i}(c)$ is unbounded below, for example in the case of log utility. Agent $i$ starts at time zero with no endowment of Arrow securities and with a tree endowment equal to a fraction $\alpha_{i}>0$ of the market portfolio, $N_{i,-1} \equiv \alpha_{i} \bar{N}$ where $\sum_{i} \alpha_{i}=1$. The agent also receives at every node $\left(t, s^{t}\right)$, an endowment of $e_{i t}\left(s^{t}\right) \geq 0$ consumption good which we will refer to as labor income.

\subsection{Agents' budget and incentive constraints}

With Arrow securities. At each node $\left(t, s^{t}\right), t<T$, agent $i$ consumes $c_{i t}\left(s^{t}\right) \geq 0$, takes long tree positions represented by a positive and finite measure over $\Delta$, denoted by $N_{i t}\left(s^{t}\right)$, and takes net positions (long minus short) $a_{i t+1}\left(s^{t}, s\right)$ in Arrow securities paying off in state $s$, for all $s \in S$. We show later, in Corollary 1, that the short-selling constraint for trees is not binding. Letting $P_{t}\left(\delta \mid s^{t}\right)$ denote the continuous price functional for trees and $Q_{t+1}\left(s^{t}, s\right)$ the price of Arrow securities at node $\left(t, s^{t}\right)$, the sequential budget constraint for $t<T$ writes:

$$
\begin{aligned}
& c_{i t}\left(s^{t}\right)+\int P_{t}\left(\delta \mid s^{t}\right) d N_{i t}\left(\delta \mid s^{t}\right)+\sum_{s} Q_{t+1}\left(s^{t}, s\right) a_{i t+1}\left(s^{t}, s\right) \\
= & e_{i t}\left(s^{t}\right)+\int\left[\delta_{t}\left(s^{t}\right)+P_{t}\left(\delta \mid s^{t}\right)\right] d N_{i t-1}\left(\delta \mid s^{t-1}\right)+a_{i t}\left(s^{t}\right),
\end{aligned}
$$

\footnotetext{
${ }^{5}$ If there is a finite number of trees, the measure-theoretic notation $d \bar{N}(\delta)$ can be replaced with $\bar{n}(\delta)$, the mass of trees with dividend stream $\delta$, and equation (1) writes as $\sum_{\delta \in \Delta} \delta_{t}\left(s^{t}\right) \bar{n}(\delta)>0$.
} 
where $a_{i 0}\left(s^{0}\right)=0$ and $N_{i,-1}=\alpha_{i} \bar{N}$ and $d N_{i t}\left(\delta \mid s^{t}\right)$ denotes the number of trees with dividend stream $\delta$ purchased by agent $i$ at node $\left(t, s^{t}\right)$. At $t=T$, the constraint writes:

$$
c_{i T}\left(s^{T}\right)=e_{i T}\left(s^{T}\right)+\int \delta_{T}\left(s^{T}\right) d N_{i T-1}\left(\delta \mid s^{T-1}\right)+a_{i T}\left(s^{T}\right) .
$$

We assume that, at each node starting at $t=1$, an agent can threaten to default, in which case its creditors obtain fraction $1-\theta$ of all long positions, for some $\theta \in(0,1) \cdot{ }^{6}$ If the agent can make a take-it-orleave-it offer to its creditors, the maximum amount it can credibly promise when selling Arrow securities is given by:

$$
a_{i t+1}^{-}\left(s^{t}, s\right) \leq(1-\theta)\left\{a_{i t+1}^{+}\left(s^{t}, s\right)+\int\left[\delta_{t+1}\left(s^{t}, s\right)+P_{t+1}\left(\delta \mid s^{t}, s\right)\right] d N_{i t}\left(\delta \mid s^{t}\right)\right\} .
$$

for all $\left(t, s^{t}\right), t<T$, and $s$, where $a_{i t+1}^{-}\left(s^{t}, s\right)=\max \left\{-a_{i t+1}\left(s^{t}, s\right), 0\right\}$ is the short position in Arrow security, and $a_{i t+1}^{+}\left(s^{t}, s\right)=\max \left\{a_{i t+1}\left(s^{t}, s\right), 0\right\}$ is the long position. Thus, we assume two-sided limited commitment in that the agent who sold Arrow securities cannot commit not to renegotiate her liabilities and the agent who bought Arrow securities cannot commit to reject take-it-or-leave-it offers.

Since (5) always holds if $a_{i t+1}\left(s^{t}, s\right) \geq 0$, it can be simplified into:

$$
-a_{i t+1}\left(s^{t}, s\right) \leq(1-\theta) \int\left[\delta_{t+1}\left(s^{t}, s\right)+P_{t+1}\left(\delta \mid s^{t}, s\right)\right] d N_{i t}\left(\delta \mid s^{t}\right)
$$

In other words, an agent's liability cannot be larger than a fraction $1-\theta$ of its tree portfolio, the maximum amount it would repay given that it can threaten to default. We will refer to equation (6) as the incentive constraint, and to the right-hand side of (6) as the agent's pledgeable income.

The incentive constraint (6) generalizes that of Chien and Lustig (2009) by allowing collateral to be imperfectly pledgeable: we assume that $\theta>0$ while Chien and Lustig assumed that $\theta=0$. While we have derived the incentive constraint (6) based on ex-post renegotiation, online Appendix XI offers an alternative micro-foundation based on limited-enforcement and cash diversion, using the optimal contracting argument

\footnotetext{
${ }^{6}$ In the appendix, we study the agent's problem and prove equilibrium existence in a more general case: we assume that the parameter $\theta$ is both agent and tree specific.
} 
of Rampini and Viswanathan (2010).

With cash on hand. Some of the analysis can be simplified with the following change of variable:

$$
W_{i t}\left(s^{t}\right) \equiv e_{i t}\left(s^{t}\right)+\int\left[\delta_{t}\left(s^{t}\right)+P_{t}\left(\delta \mid s^{t}\right)\right] d N_{i t-1}\left(\delta \mid s^{t-1}\right)+a_{i t}\left(s^{t}\right)
$$

In words, $W_{i t}\left(s^{t}\right)$ represents the agent's cash-on-hand: the combined value of the endowment, the tree portfolio and the Arrow security payoff. One advantage of the cash-on-hand formulation is to simplify notations by suppressing any explicit reference to Arrow securities. Indeed, with cash-on-hand, the sequential budget constraint becomes:

$$
\begin{aligned}
& c_{i t}\left(s^{t}\right)+\int P_{t}\left(\delta \mid s^{t}\right) d N_{i t}\left(\delta \mid s^{t}\right)+\sum_{s} Q_{t+1}\left(s^{t}, s\right) W_{i t+1}\left(s^{t}, s\right) \\
= & W_{i t}\left(s^{t}\right)+\sum_{s} Q_{t+1}\left(s^{t}, s\right) e_{i t+1}\left(s^{t}, s\right)+\sum_{s} Q_{t+1}\left(s^{t}, s\right) \int\left[\delta_{t+1}\left(s^{t}, s\right)+P_{t+1}\left(\delta \mid s^{t}, s\right)\right] d N_{i t}\left(\delta \mid s^{t}\right),
\end{aligned}
$$

for all $\left(t, s^{t}\right)$, and with the convention that time $T+1$ variables and time $T$ tree prices are equal to zero. Likewise, the incentive constraint (6) can be written:

$$
W_{i t+1}\left(s^{t}, s\right) \geq e_{i t+1}\left(s^{t}, s\right)+\theta \int\left[\delta_{t+1}\left(s^{t}, s\right)+P_{t+1}\left(\delta \mid s^{t}, s\right)\right] d N_{i t}\left(\delta \mid s^{t}\right),
$$

for all $\left(t, s^{t}\right), t<T$, and $s$. Equation (8) states that agents' cash-on-hand in all successor nodes must be larger than the non-pledgeable income stemming from their labor endowment and tree payoff. This limits an agent's ability to hold trees whose payoff is high in the states in which she is constrained.

\subsection{Definition of equilibrium}

A price system is some $(P, Q)$, where $P \equiv\left\{P_{t}\left(\delta \mid s^{t}\right), 0 \leq t<T, s^{t} \in S^{t}\right\}$ is a sequence of positive and continuous price functionals for trees, and $Q \equiv\left\{Q_{t+1}\left(s^{t}, s\right), 0 \leq t<T, s^{t} \in S^{t}, s \in S\right\}$ is a sequence of Arrow security prices. Given $(P, Q)$, an agent chooses plans for consumption, $c_{i}=\left\{c_{i t}\left(s^{t}\right), 0 \leq t \leq\right.$ $\left.T, s^{t} \in S^{t}\right\}$, and for tree portfolios, $N_{i}=\left\{N_{i t}\left(s^{t}\right), 0 \leq t<T, s^{t} \in S^{t}\right\}$. A plan for consumption and tree 
portfolios, $\left(c_{i}, N_{i}\right)$, is budget feasible and incentive compatible if there exists some plan for cash-on-hand $W_{i}=\left\{W_{i t}\left(s^{t}\right), 0 \leq t \leq T, s^{t} \in S^{t}\right\}$ such that $\left(c_{i}, N_{i}, W_{i}\right)$ satisfies the budget constraint (7) at all nodes, the incentive constraint (8) at all nodes, and the initial condition:

$$
W_{i 0}\left(s^{0}\right)=e_{i 0}\left(s^{0}\right)+\alpha_{i} \int\left[\delta_{0}\left(s^{0}\right)+P_{0}\left(\delta \mid s^{0}\right)\right] d \bar{N}(\delta)
$$

The agent's problem is to choose a budget feasible and incentive compatible plan, $\left(c_{i}, N_{i}\right)$, in order to maximize the intertemporal utility (2).

An allocation is a collection $\left(c_{i}, N_{i}\right)_{i \in I}$ of plans for consumption and tree portfolio. An allocation is feasible if, at all nodes $\left(t, s^{t}\right)$ :

$$
\begin{aligned}
\sum_{i} c_{i t}\left(s^{t}\right) & =\sum_{i} e_{i t}\left(s^{t}\right)+\sum_{i} \int \delta_{t}\left(s^{t}\right) d N_{i t-1}\left(\delta \mid s^{t-1}\right) \\
\sum_{i} N_{i t}\left(s^{t}\right) & =\bar{N} .
\end{aligned}
$$

The feasibility condition for trees (11) states that the demand for dividend stream $\delta, \sum_{i} d N_{i t}\left(\delta \mid s^{t}\right)$, is equal to the supply, $d \bar{N}(\delta)$.

An equilibrium is a price system $(P, Q)$ and a feasible allocation $\left(c_{i}, N_{i}\right)_{i \in I}$ such that, for all $i \in I,\left(c_{i}, N_{i}\right)$ solves the problem of agent's $i$ given $(P, Q)$.

This definition is formulated in the spirit of a classical time-zero Arrow-Debreu equilibrium, in the sense that it suppresses any explicit reference to agents' positions in Arrow securities. ${ }^{7}$ There is one important difference however, which sets our model apart from earlier work in the endogenous incomplete market literature, such as Alvarez and Jermann (2000), Chien and Lustig (2009) and Gottardi and Kubler (2015). In the Arrow-Debreu equilibria defined in these earlier works, agents do not explicitly trade trees: indeed noarbitrage implies that it is equivalent for agents to only trade claims to consumptions at all future nodes. Our definition, in contrast, must be explicit about agents' trades in trees. This is because trees are imperfectly pledgeable, implying, as shown below, that standard no-arbitrage relationships do not apply and trading

\footnotetext{
${ }^{7}$ See, for example, Chapter 8 in Ljungqvist and Sargent (2012). It is routine to verify that the definition is equivalent to the corresponding one with Arrow securities. For example, using the sequential budget constraints (3), one can recover agents' implied Arrow securities positions, and verify that the market for Arrow securities clears.
} 
trees is no longer equivalent to trading consumption claims. ${ }^{8}$

\section{Equilibrium Analysis}

\subsection{No-arbitrage relationships}

We first establish key no-arbitrage relationships that have to hold in our setting:

Lemma 1 Let $(P, Q)$ and $\left(c_{i}, N_{i}\right)_{i \in I}$ be an equilibrium. Then:

1. The price of consumption is strictly positive at all $\left(t, s^{t}\right), t<T$ :

$$
Q_{t+1}\left(s^{t}, s\right)>0
$$

2. Trees are priced at most at the value of their total payoff at all $\left(t, s^{t}\right), t<T$ :

$$
P_{t}\left(\delta \mid s^{t}\right) \leq \sum_{s} Q_{t+1}\left(s^{t}, s\right)\left[\delta_{t+1}\left(s^{t}, s\right)+P_{t+1}\left(\delta \mid s^{t}, s\right)\right]
$$

$\bar{N}$-almost everywhere in $\Delta$.

3. Trees are priced at least at the value of their pledgeable payoff:

$$
P_{t}\left(\delta \mid s^{t}\right) \geq(1-\theta) \sum_{s} Q_{t+1}\left(s^{t}, s\right)\left[\delta_{t+1}\left(s^{t}, s\right)+P_{t+1}\left(\delta \mid s^{t}, s\right)\right]
$$

everywhere in $\Delta$, with a strict inequality if the continuation dividend stream is non zero.

For the first no-arbitrage relationship, suppose that the price of consumption were zero for some $\left(t, s^{t}\right)$ : then all agents would find it optimal to increase their consumption at that node, violating the market clearing condition for consumption.

\footnotetext{
${ }^{8}$ To be clear, Chien and Lustig (2009) and Gottardi and Kubler (2015) define Arrow Debreu equilibria differently from us. In particular, they do not re-define incentive constraints based on a notion of cash-on-hand, but instead they show how to replace the collateral constraints by what they call "solvency constraints": namely at all nodes, the present value of consumption must be larger than that of the labor endowment. The key point is that, in Chien and Lustig (2009) and Gottardi and Kubler (2015), agents' tree portfolios do not enter these solvency constraints. We can derive solvency constraints in our setting as well, by iterating the incentive constraints (8) forward. But, in contrast to Chien and Lustig (2009) and Gottardi and Kubler (2015), imperfect pledgeability implies that these solvency constraints now depend on agents' tree portfolios.
} 
For the second no-arbitrage relationship, suppose that at node $\left(t, s^{t}\right)$, the price of some tree in positive supply were strictly larger than the present value of its total future payoffs. Then all agents holding this tree could sell it and purchase instead a replicating portfolio of Arrow securities, making a risk-free profit without violating their incentive constraint: indeed equation (8) shows that replacing a tree with a replicating portfolio of Arrow securities keeps cash-on-hand the same but reduces the non-pledgeable income stemming from the tree payoff. Hence, if the second no-arbitrage relationship did not hold, the market could not clear. ${ }^{9}$

Finally, for the third no-arbitrage relationship suppose that at some node $\left(t, s^{t}\right)$, the price of some tree with non-zero continuation dividend stream were lower than the value of its pledgeable future payoffs. Then an agent could finance the purchase of the tree by selling a replicating portfolio of its pledgeable payoff, and consume the non pledgeable payoff next period, which must be strictly positive in at least some state. This would imply infinite demand at some node and violate the market clearing conditions.

While (13) also holds in frictionless markets, (14) is specific to our model as it involves the parameter $\theta$ reflecting that trees' payoffs are imperfectly pledgeable. Taken together, the second and third no-arbitrage relationships show that, in our model, the Law of One Price may only fail in one direction: trees can be priced below, but not above, the portfolio of Arrow securities replicating their payoff. Below we show that strict violations of the Law of One Price arise in equilibrium.

\subsection{Equilibrium existence}

Establishing existence is challenging in part because some equilibrium objects are infinite-dimensional: tree portfolios are represented by finite measures and, correspondingly, tree prices are represented by continuous functionals. Moreover, since prices enter incentive constraints, we cannot apply existence arguments based on Welfare Theorems (Negishi, 1960). Instead, we use the classical price-player proof of Arrow and Debreu (1954), with two changes. First, since agents face incentive constraints that depend on prices, we must revisit the proof that constraint sets are lower hemi-continuous with respect to prices. Second, the constraint set of the price player must allow deviations from the Law of One Price and, correspondingly, its objective must account for the arbitrage revenues generated by agents' net trades in the market for trees (see Appendices

\footnotetext{
${ }^{9}$ Notice that this reasoning only applies to trees in positive supply, which is why it only holds almost everywhere according to $\bar{N}$. For trees in zero supply, the only restriction is that the price must be large enough so that agents do not find optimal to hold them.
} 
A.1 and A.4). One advantage of the "cash on hand" formulation of budget and incentive constraints is to help coping with these difficulties. ${ }^{10}$

The proof of existence proceeds in two steps. We first consider tree supplies with finite support, a simpler case because it can be handled with finite-dimensional vector space methods. In particular, we can first determine finitely many tree prices, in the support of the supply distribution, and then provide a natural extension of this price vector to a continuous price functional valuing all dividend streams in $\Delta$. Next, we rely on the fact that the set of positive measures on $\Delta$ with finite support is dense in the set of all positive measures on $\Delta$, endowed with the weak topology. Given a sequence of discrete measures converging weakly to any arbitrary finite measure $\bar{N}$, and an associated sequence of equilibria, we can extract a subsequence converging to an equilibrium given supply $\bar{N}$. In sum:

Theorem 1 There exists an equilibrium.

While our analysis so far relied on the assumption that agents cannot short trees, it turns out that this constraint is not binding. Suppose indeed that, in addition to Arrow securities, agents are allowed to short trees: then agent $i$ 's tree portfolio can be written as the difference between two positive measures, $N_{i}=N_{i}^{+}-N_{i}^{-}$, where $N_{i}^{+}$represents long and $N_{i}^{-}$short positions. Since short-positions are liabilities, they must be subject to some incentive constraint. Going through the same reasoning as before, we obtain:

$$
\begin{aligned}
a_{i t+1}^{-}\left(s^{t}, s\right) & +\int\left[\delta_{t+1}\left(s^{t}, s\right)+P_{t+1}\left(\delta \mid s^{t}, s\right)\right] d N_{i t}^{-}\left(\delta \mid s^{t}\right) \\
\leq & (1-\theta)\left\{a_{i t+1}^{+}\left(s^{t}, s\right)+\int\left[\delta_{t+1}\left(s^{t}, s\right)+P_{t+1}\left(\delta \mid s^{t}, s\right)\right] d N_{i t}^{+}\left(\delta \mid s^{t}, s\right)\right\} .
\end{aligned}
$$

We then establish:

Corollary 1 An equilibrium arising when agents can only short Arrow securities remains an equilibrium when agents can short both trees and Arrow securities.

To see why the result obtains, consider an equilibrium when agents can only short Arrow securities. In equilibrium, as stated in Lemma 1, trees are priced below (but not above) replicating portfolios of Arrow

\footnotetext{
${ }^{10}$ Indeed, by suppressing the need to clear the market for Arrow securities this formulation makes it easier to formulate Walras Law and define the price-player objective. Moreover, cash-on-hand can be used as state variable for a recursive proof of lower hemi-continuity.
} 
securities. Hence, agents do not find it optimal to short trees: they prefer instead to short replicating portfolios of Arrow securities. ${ }^{11}$

Of course, while tree short selling constraints do not bind, incentive constraints could bind. We now examine conditions under which it is the case. Let $(Q, c)$ be the price system and consumption allocation of a complete market equilibrium, i.e., with complete market and no incentive constraints. Now consider a corresponding economy with incentive constraints. ${ }^{12}$ We say that $(Q, c)$ is $I C$-implementable if there exists an equilibrium with incentive constraints, $(\hat{P}, \hat{Q})$ and $(\hat{c}, \hat{N})$ such that $Q=\hat{Q}$ and $c=\hat{c}$. In Appendix D we derive necessary and sufficient conditions for IC implementability, leading to:

Proposition 1 Let $\theta^{\star}$ be the largest $\theta$ such that a given complete market equilibrium is IC-implementable:

1. $\theta^{\star}>0$ if $e$ is small and Inada conditions hold for all agents;

2. $\theta^{\star}<1$ if $\bar{N}$ is small, $e \gg 0$ and marginal rate of substitutions evaluated at e are not equalized;

3. $\theta^{\star}<1$ if agents have heterogenous CRRA utility, $e$ is small, and there is one tree;

4. $\theta^{\star}$ is weakly increasing in tree supply dispersion.

Notice that if an allocation is IC implementable for a given $\theta$, it remains IC implementable if $\theta$ is lower.

The first two points of the proposition highlight that complete market outcomes obtain when the supply of collateral is sufficiently large and pledgeable.

Under the assumptions stated in the first point, agents want their cash-on-hand to remain large enough at every node: otherwise, since they do not have much labor income, they would be forced to consume little, which is not optimal under Inada conditions. This means that agents do not find it optimal to issue large liabilities. Hence, incentive constraints are slack as long as trees are sufficiently pledgeable, i.e., for all $\theta$ small enough.

\footnotetext{
${ }^{11}$ An earlier draft of this paper showed a stronger result. Namely, in a two-periods version of the model, any equilibrium with short-selling is essentially equivalent to an equilibrium with no short-selling, with identical consumption allocation and price system.

${ }^{12}$ Formally, in a corresponding economy with incentive constraints, agents have the same preference and consumption-good endowment as in the complete-market economy, that is, at all nodes, the sum of their labor and tree income endowment is equal to their consumption endowment in the complete-market economy. Notice that there are many possible such economies, differing in terms of their pledgeability parameter and of the break down of consumption good endowment between labor and tree income.
} 
In the second point, intertemporal marginal rates of substitution evaluated at $e$ are not equalized, so agents would benefit from risk sharing to smooth consumption. But such risk sharing is ruled out by the scarcity of collateral, $\bar{N}(\Delta) \simeq 0$.

The last two points of the proposition emphasize that the implementability of complete market outcomes not only depend on size and pledgeability of the collateral supply, but also on its distribution.

To gain intuition about the third point recall that, in a complete market equilibrium with heterogenous CRRA utility, agents' consumption shares are not constant: they depend on the current realization of the aggregate endowment. But if there is just one tree and little labor income, aggregate ressources are all bundled in a single tree. As a result, when agents trade the tree, they can only attain approximately constant consumption shares. Hence agents need to issue liabilities to attain their complete-market state-dependent consumption shares. Correspondingly, a complete market equilibrium is not IC-implementable as long as $\theta$ is close enough to one, i.e., as long as agents cannot issue much liabilities.

For the fourth point, suppose as a special case that $e=0$, and that the distribution of tree supply is maximally dispersed. Specifically, assume that all trees in positive supply are Arrow securities, in the sense that they only pay dividend at one node. Then, it is clear that the complete market outcome is IC-implementable: all agents can synthetize their complete-market consumption profile by purchasing these Arrow trees only, while respecting market clearing in the aggregate. Our proof generalizes this example: it shows that IC implementation becomes easier if one increases supply dispersion by breaking up existing trees into replicating portfolios.

\subsection{First-order conditions}

We now state the first-order necessary and sufficient conditions for the agent's problem (the formal derivation is in Appendix A.3). Let $\lambda_{i t}\left(s^{t}\right) \geq 0$ denote the multiplier for the sequential budget constraint (7) at node $\left(t, s^{t}\right)$ and $\mu_{i t+1}\left(s^{t}, s\right) \geq 0$ the multiplier for the incentive constraint (8). The first-order conditions with 
respect to $c_{i t}\left(s^{t}\right)$ and $W_{i t+1}\left(s^{t}, s\right)$ write:

$$
\begin{aligned}
& \beta^{t} \pi_{t}\left(s^{t}\right) u_{i}^{\prime}\left(c_{i t}\left(s^{t}\right)\right)=\lambda_{i t}\left(s^{t}\right) \\
& \lambda_{i t+1}\left(s^{t}, s\right)+\mu_{i t+1}\left(s^{t}, s\right)=\lambda_{i t}\left(s^{t}\right) Q_{t+1}\left(s^{t}, s\right) .
\end{aligned}
$$

where we have assumed strictly positive consumption for simplicity. The first condition states that the agent chooses consumption to equate marginal utility with marginal cost, which is equal to the multiplier of the budget constraint, $\lambda_{i t}\left(s^{t}\right)$. The second condition equates the marginal value and marginal cost of increasing cash-on-hand next period, $W_{i t+1}\left(s^{t}, s\right)$. It reveals that the marginal value of increasing cash-on-hand next period has two components: it relaxes both the budget constraint, with marginal value $\lambda_{i t+1}\left(s^{t}, s\right)$, and the incentive constraint, with marginal value $\mu_{i t+1}\left(s^{t}, s\right)$. The intuition for the latter is that higher cash-on-hand reduces the agent's incentive to default. Combining the two we obtain that

$$
Q_{t+1}\left(s^{t}, s\right)=\beta \pi_{t+1}\left(s \mid s^{t}\right) \frac{u_{i}^{\prime}\left(c_{i t+1}\left(s^{t}, s\right)\right)}{u_{i}^{\prime}\left(c_{i t}\left(s^{t}\right)\right)}+\frac{\mu_{i t+1}\left(s^{t}, s\right)}{\lambda_{i t}\left(s^{t}\right)}
$$

Condition (18) is familiar from the limited-commitment literature (see, e.g. Alvarez and Jermann, 2000), and means that Arrow securities are priced by those agents whose incentive constraints are not binding, for example, agents who are long Arrow securities. When an agent's incentive constraint is not binding, $\mu_{i t+1}\left(s^{t}, s\right)=0$ and the agent's intertemporal marginal rate of substitution is equal to the corresponding Arrow security price. By contrast, for the agents whose incentive constraint is binding, $\mu_{i t+1}\left(s^{t}, s\right)>0$ and the agent's intertemporal marginal rate of substitution is strictly lower than the corresponding Arrow security price. This, however, does not prompt the agent to sell the Arrow security because this would violate her incentive constraint.

New to our setting is the first-order condition with respect to tree holdings, which can be stated as:

$$
\sum_{s} Q_{i t+1}\left(s^{t}, s\right)\left[\delta_{t+1}\left(s^{t}, s\right)+P_{t+1}\left(\delta \mid s^{t}, s\right)\right] \leq P_{t}\left(\delta \mid s^{t}\right),
$$


with an equality if the agent holds the tree, that is, if $d N_{i t}\left(\delta \mid s^{t}\right)>0$, and where

$$
Q_{i t+1}\left(s^{t}, s\right) \equiv(1-\theta) Q_{t+1}\left(s^{t}, s\right)+\theta \beta \pi_{t+1}\left(s \mid s^{t}\right) \frac{u_{i}^{\prime}\left(c_{i t+1}\left(s^{t}, s\right)\right)}{u_{i}^{\prime}\left(c_{i t}\left(s^{t}\right)\right)}
$$

is the private valuation of agent $i$ for an Arrow security paying off in state $s$ at time $t+1$. The economic interpretation is that the payoff has both a pledgeable and a non-pledgeable component, which are valued differently. While the agent values the pledgeable component using the price of Arrow securities (first term on the right-hand side of (20)), it values the non-pledgeable component using its own intertemporal marginal rate of substitution (second term). To the extent that different agents' incentive constraints bind in different states, marginal rates of substitution and therefore private valuations differ across agents.

\subsection{Segmentation}

Optimal payoff sets. For each node $\left(t, s^{t}\right)$, each agent $i$, and any vector of one-period ahead payoff $x \in \mathbb{R}_{+}^{S}$, the left-hand side of equation (19) defines a private valuation operator:

$$
Q_{i t+1}\left(s^{t}\right) \cdot x=\sum_{s} Q_{i t+1}\left(s^{t}, s\right) x(s)
$$

the dot-product between the vector of private valuations for Arrow securities, and the vector of one-period ahead payoffs. Correspondingly, there is a set of one-period ahead payoffs for which agent $i$ is the best holder:

$$
X_{i t}\left(s^{t}\right) \equiv\left\{x \in \mathbb{R}_{+}^{S}: Q_{i t+1}\left(s^{t}\right) \cdot x \geq Q_{j t+1}\left(s^{t}\right) \cdot x, \text { for all } j\right\}
$$

Agent $i$ only holds trees whose vector of one-period ahead payoffs (i.e., the vector of the cum-dividend price) lies in $X_{i t}\left(s^{t}\right)$. In what follows, we will refer to $X_{i t}\left(s^{t}\right)$ as the optimal payoff set of agent $i$. We show below that, in general, because agents have different private valuations, they have distinct optimal payoff sets, and so hold different trees in equilibrium. Hence, the tree market is endogenously segmented. ${ }^{13}$

\footnotetext{
${ }^{13}$ Of course, for trees, one-period ahead payoffs depend on future prices and so are endogenous. In Section 3.6, we show how to characterize segmentation in the set of dividend streams as opposed to the set of payoffs.
} 
Characterizing the collection of optimal payoff sets, $\left\{X_{i t}\left(s^{t}\right), i=1, \ldots, I\right\}$, is a classical problem in Optimal Transport theory, studied in Chapter 5 of Galichon (2016): the problem of drawing "power-diagrams." Although this problem does not have an analytical solution in general, it has simple geometrical properties. Moreover, numerical calculations are facilitated by the observation that optimal payoff sets solve a convex optimization problem. See Online Appendix IX for more mathematical details. The proposition below states some key properties of optimal payoff sets.

Proposition 2 The collection of optimal payoff sets $\left\{X_{i t}\left(s^{t}\right), i=1, \ldots, I\right\}$, has the following properties:

1. Optimal payoff sets are convex polyhedral cones covering $\mathbb{R}_{+}^{S}$.

2. For any two pairs of agents, the optimal payoff sets are either identical $\left(X_{i t}\left(s^{t}\right)=X_{j t}\left(s^{t}\right)\right)$ or have disjoint interiors $\left(\dot{X}_{i t}\left(s^{t}\right) \cap \stackrel{\circ}{X}_{j t}\left(s^{t}\right)=\emptyset\right)$.

3. If no incentive constraint binds in the next period, then $X_{i t}\left(s^{t}\right)=\mathbb{R}_{+}^{S}$ for all $i$. Otherwise, if there exists an agent $i$ whose incentive constraint binds in some state in the next period, then $X_{i t}\left(s^{t}\right)$ is a strict subset of $\mathbb{R}_{+}^{S}$ and there exists another agent $j$ such that $\stackrel{\circ}{i t}_{i t}\left(s^{t}\right) \cap \stackrel{\circ}{X}_{j t}\left(s^{t}\right)=\emptyset$.

The first bullet point of the proposition follows because optimal payoff sets are defined by linear inequalities. The payoff vector of a tree is represented by a point in $\mathbb{R}_{+}^{S}$. The direction of the vector represents the tree's risk exposure, i.e., the distribution of its payoff across states. That optimal payoff sets are cones means that if an asset is in the set, any asset with proportional payoff, i.e. with the same risk exposure, is also in the set. To illustrate this, Figure 2 displays a convex polyhedral cone in the payoff space when there are three states. The rightmost facet of the polyhedron is the intersection of the cone with the simplex, which will be useful for the analysis.

The interpretation of the second bullet point is the following. If two agents have the same private valuations, $Q_{i t+1}\left(s^{t}, s\right)=Q_{j t+1}\left(s^{t}, s\right)$ for all $s \in S$, then they must have the same optimal payoff sets. Otherwise, if two agents have different private valuations, the set of payoffs for which they have the same private valuations is an hyperplane, thus its interior is zero.

Finally, we turn to the third bullet point. If no incentive constraint binds, all private valuations are the same, so equation (22) implies that agents' have the same optimal payoff sets, which must be $\mathbb{R}_{+}^{S}$. If an 


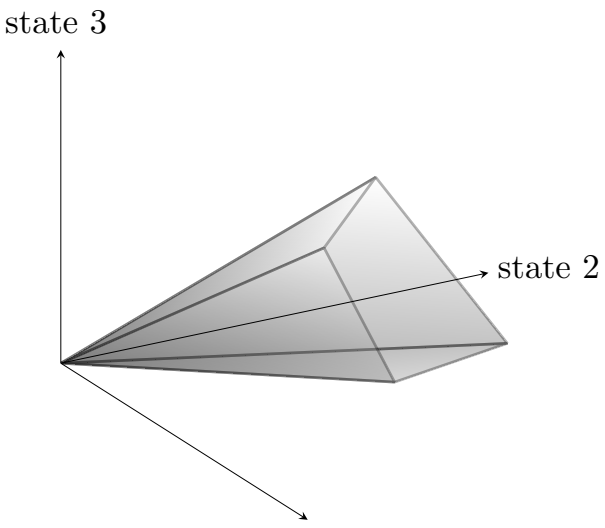

state 1

Figure 2: A polyhedral cone with three successor states.

incentive constraint binds for some agent $i$ in some state $s$, it means agent $i$ has large liabilities in state $s$, created by short positions in Arrow securities paying off in this state. By market clearing, there is another agent $j \neq i$ who has long positions in these Arrow securities and therefore no liabilities in state $s$. Thus, agent $j$ 's incentive constraint is slack in state $s$. Hence, agents $i$ and $j$ have different private valuations. In particular, agent $i$ has a lower valuation than $j$ for trees with large payoff in state $s$. Therefore, agents $i$ and $j$ have different optimal payoff sets and correspondingly different holdings, i.e., there is segmentation.

To illustrate how segmentation in the market for trees is related to the demand for Arrow securities, consider agents who want to hedge against the risk of a given state $s$ occurring. These agents purchase Arrow securities paying off in that state. But the supply of Arrow securities is limited by incentive constraints, hence insurance is imperfect and these agents have high marginal utility in that state and therefore high private valuations for trees with a high payoff in that state. Therefore, they buy trees lying in a cone that is close to the axis corresponding to that state.

While we have a characterization of optimal payoff sets given the vector of private valuations, it is difficult to solve analytically the general equilibrium problem of finding the private valuations. To sidestep this difficulty, we consider the limiting case of an economy with no collateral, $\bar{N}=0 .{ }^{14}$ In that case, the marginal rates of substitution are easy to characterize because the agents just consume their labor endowments. Equipped with those marginal rate of substitutions, we can solve the Optimal Transport

\footnotetext{
${ }^{14}$ The economy with no collateral does not satisfies our maintained assumption (1) that the aggregate dividend is strictly positive at all nodes, so Theorem 1 does not apply. However, it is easy to show by hand that an equilibrium exists, that the allocation is unique, and that an equilibrium price system is obtained from the same first-order conditions as in the rest of the paper. See Online Appendix VIII.
} 
problem and characterize the collection of optimal payoff sets. ${ }^{15}$ Of course, when $\bar{N}=0$, there are no trees around that agents can use as collateral to issue Arrow securities. But we establish a continuity argument in Online Appendix VIII: the $\bar{N}=0$ marginal rates of substitution, as well as the corresponding optimal payoff sets, approximate those arising in an economy with little collateral, $\bar{N} \simeq 0 .{ }^{16}$ This implies that, when $\bar{N} \simeq 0$, agents will purchase the trees whose payoffs lie in the interior of their $\bar{N}=0$ optimal payoff set, and use them as collateral to sell Arrow securities whose $\bar{N}=0$ price is strictly larger than their marginal rate of substitution.

A first parametric example. We consider an economy populated by many log utility agents, who are hit by heterogeneous endowment growth shocks i.i.d. over time. Suppose there are three states (see Online Appendix IX for assumptions and computations). To represent graphically the collection of optimal payoff sets, we plot their intersection with the simplex, as shown in Figure 3. These intersections fully characterize the optimal payoff sets, since these are cones. The figure reveals that some agents only hold assets near corners, i.e., assets which are approximately Arrow securities. These agents have the highest intertemporal marginal rate of substitutions corresponding to that state. Other agents hold assets away from corners. These agents do not have a maximal intertemporal marginal rate of substitution for any state, and so they do not hold any Arrow security. However, they can be the best holders of some other interior trees, with positive payoff in all states. In equilibrium, these agents buy these interior trees and use them as collateral to sell Arrow securities conditional on all states. ${ }^{17}$

A second parametric example. Our second example provides a full characterization of equilibrium allocations in an economy log utility agents and with two states. We parameterize the endowment growth

\footnotetext{
${ }^{15}$ In an economy with no collateral $\bar{N}=0$, borrowing constraints are "maximally tight" in the sense of Krusell, Mukoyama and Smith (2011). As a result, the allocation becomes trivial: it is "hand-to-mouth." This property makes an economy with no collateral as tractable as a representative agent economy: optimal payoff sets and asset prices can be characterized in closed form.

${ }^{16}$ To be clear, our arguments establish continuity for allocations and price systems, and upper hemi continuity for optimal payoff sets.

${ }^{17}$ Figure 3 also suggests that optimal payoff sets have other geometrical properties than the ones noted in Proposition 2 ,for example they are "face to face". Indeed, it turns out that the properties of Proposition 2 are only necessary: the geometry of power diagrams places additional restrictions on optimal payoff sets (see Aurenhammer, 1987a,b; Ziegler, 1995, and our summary in Online Appendix IX) but these do not have obvious economic interpretations.
} 


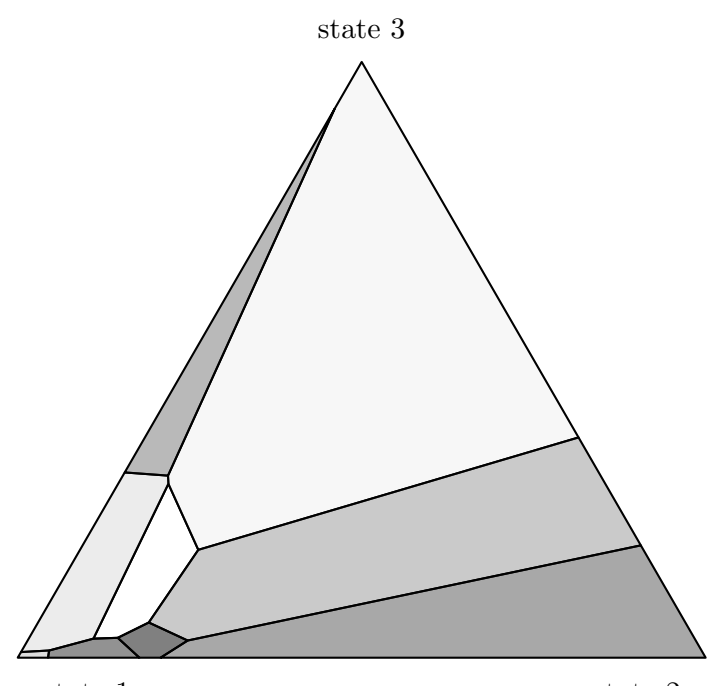

state 1

state 2

Figure 3: First parametric example: intersections of optimal payoff sets with the simplex.

of agents by $\alpha_{1}=0<\alpha_{2}<\ldots<\alpha_{I}$. In state 1 occurring with probability $\pi_{1}$, the endowment growth is

$$
g_{1}(\alpha)=g\left(1+k_{1} \alpha\right)^{-\phi}
$$

while in state 2 occurring with probability $\pi_{2}$, it is

$$
g_{2}(\alpha)=g\left(1-k_{2} \alpha\right)^{-\phi},
$$

where $g>0,0<k_{1} \pi_{1}<k_{2} \pi_{2}$ and $k_{2} \alpha_{I}<1$. For all agents, $g_{1}(\alpha) \leq g_{2}(\alpha)$, meaning that $s=1$ is the "bad state" while $s=2$ is the "good state". Agents with higher $\alpha$ have a higher exposure to the risk of the bad state. The payoff of a tree in the simplex is $(x, 1-x)$ where $x$ is the payoff in the bad state.

Proposition 3 In the parametric example above, if $\phi \gamma<1$, there exists a strictly increasing sequence $x_{0}=0<x_{1}<\ldots<x_{I}=1$ such that the intersection of the optimal payoff set of agent $\alpha_{i}$ with the simplex is $X_{i}=\left[x_{i-1}, x_{i}\right]$.

Therefore, agents with higher exposure to risk of the bad state (with higher $\alpha$ ) hold trees with large payoffs in the bad state, which hedge them better. Extreme agents $\alpha_{1}=0$ and $\alpha_{I}$ hold assets in the neighborhood 


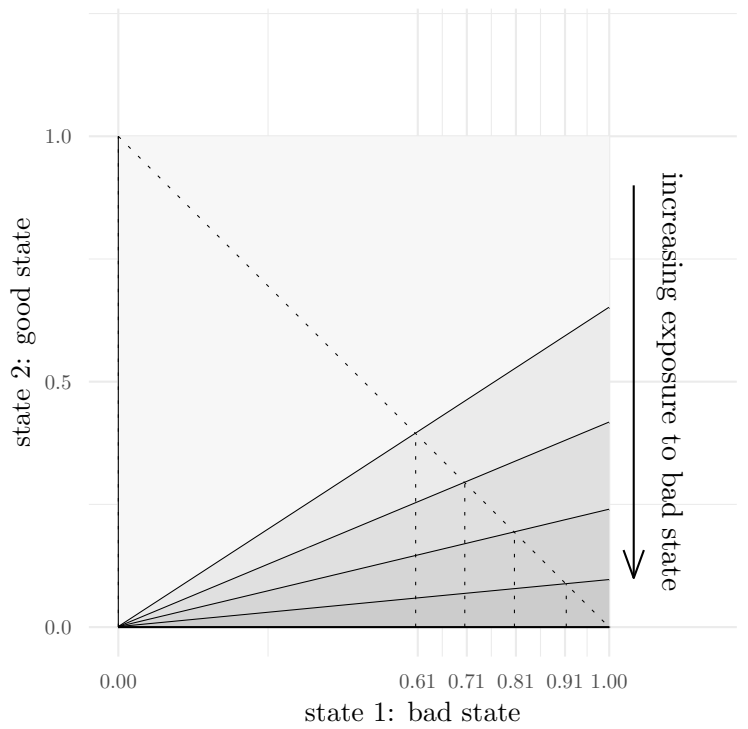

Figure 4: Second parametric example: intersection of optimal payoff sets with the unit square.

of Arrow securities, while intermediate agents hold other assets. Figure 4 represents the optimal payoff sets as shaded areas, in an example economy with $I=5$ agents. The cones further to the northwest correspond to lower values of $\alpha$. The sequence $x_{i}$ is represented as the tick labels of the $x$ axis. ${ }^{18}$.

\subsection{Asset pricing}

From equation (19), it follows that the recursion

$$
P_{t}\left(\delta \mid s^{t}\right)=\max _{i} \sum_{s} Q_{i t+1}\left(s^{t}, s\right)\left[\delta_{t+1}\left(s^{t}, s\right)+P_{t+1}\left(\delta \mid s^{t}, s\right)\right]
$$

defines an equilibrium price functional for all trees $\delta \in \Delta \cdot{ }^{19}$ Equation (25) means that the price of the tree is the maximum of the private valuations of all agents for that tree. In this section we study the implications of this asset pricing formula.

\footnotetext{
${ }^{18}$ Online Appendix X covers the other case. We show that when $\phi \gamma \geq 1$, assets are only held by extreme agents, $\alpha_{1}$ and $\alpha_{I}$. In that case, the optimal payoff sets of intermediate agents are either empty or singleton (i.e., have an empty interior), two properties consistent with Proposition 2.

${ }^{19}$ In all equilibria, this equation holds with equality for trees in positive supply, and otherwise with inequality. We assume from now on that it holds with equality for all trees in $\Delta$, which is natural and without much loss of generality: indeed, this equation determines the equilibrium price of tree $\delta$ as soon as it s supply outstanding is arbitrarily small.
} 
Deviations from the Law of One Price. Because the pricing operator (25) is convex and linearly homogenous in payoffs, a tree must be priced below any replicating portfolio of long positions, comprised of trees or Arrow securities. For example, considering three trees with time $t+1$ payoffs $x, y$, and $z=x+y$, respectively, we have

$$
\max _{i} Q_{i t+1} \cdot z \leq \max _{i} Q_{i t+1} \cdot x+\max _{i} Q_{i t+1} \cdot y
$$

that is, $z$ is valued below its replicating portfolio $x+y$. The inequality is strict if there is no agent who has the highest valuation for both $x$ and $y$. This is stated more generally in the next proposition.

Proposition 4 Consider tree $\delta$ in node $\left(t, s^{t}\right)$ and a replicating portfolio $M$, that is,

$$
\delta_{t+1}\left(s^{t}, s\right)+P_{t+1}\left(\delta \mid s^{t}, s\right)=\int\left[\delta_{t+1}^{\prime}\left(s^{t}, s\right)+P_{t+1}\left(\delta^{\prime} \mid s^{t}, s\right)\right] d M\left(\delta^{\prime}\right)
$$

for all $s \in S$. If there exists no agent whose optimal payoff set includes the payoffs of (almost) all assets in the replicating portfolio, then tree $\delta$ is priced strictly below its replicating portfolio:

$$
P_{t}\left(\delta \mid s^{t}\right)<\int P_{t}\left(\delta^{\prime} \mid s^{t}\right) d M\left(\delta^{\prime}\right)
$$

Note that the replicating portfolio $M$ can include trees, or Arrow securities, or both. The economic intuition of Proposition 4 is that a tree is a bundle of risks that cannot be traded separately from one another, whereas the portfolio of securities with the same payoff as the tree is a bundle of risks that can be traded separately. If there is no agent whose valuation for all the securities in that portfolio is the highest among agents, then no agent wants to hold all the securities in the replicating portfolio and bear the corresponding bundle of risks. Instead, all agents prefer to pick and choose among the risks in the bundle, retaining only those they want to bear. Therefore, the tree is priced below its replicating portfolio, that is, there is a basis.

A specific implication of our model is that the basis always goes in the same direction. Consistent with the no-arbitrage relationships in Lemma 1, the price of a tree can be lower than that of the replicating portfolio of trees and/or Arrow securities, but it cannot be higher. If it was higher, an agent holding the 
tree could sell it and buy the replicating portfolio. That arbitrage trade would be feasible because i) market clearing implies there is at least one agent holding the tree, and ii) replacing a tree by its replicating portfolio does not tighten the incentive constraint. In contrast with i), when the price of the tree is lower than that of the replicating portfolio, there does not exist an agent holding the replicating portfolio (since holding that portfolio is dominated). Hence arbitrage trades would require the issuance of liabilities, which would tighten the incentive constraint (in contrast with ii)).

The literature has shown that pledgeable payoffs should be priced higher than non-pledgeable ones, which also holds in our model as is clear from equation (20). Thus, the literature has identified the collateral premium, i.e., pledgeable trees should be priced higher than non-pledgeable ones. In contrast, we show there is a basis between a tree and a replicating portfolio of identically pledgeable securities, to the extent that the payoffs of these securities are differently bundled across states than the tree.

For example, a convertible bond is a bundle of a straight bond and a call option on the issuer's stock. In the language of our model, a convertible bond is a tree with the same payoff as a combination of another tree (the straight bond) with a portfolio of Arrow securities (the call option). Our model implies that, if there are no agents who hold simultaneously the straight bond and the call, then the convertible bond should be priced strictly below the price of the straight bond plus the price of the call. In line with our theory, convertible bonds are in fact priced below the replicating portfolio. This deviation from the Law of One Price is at the root of a popular hedge fund strategy ("convertible arbitrage"), which consists in stripping the convertible bond (Mitchell and Pulvino, 2012). Hedge funds buy the convertible bond, issue the set of securities that replicate the convertible bond, and sell the different securities to different clienteles: debt securities are distributed through prime brokers to money market funds and other buyers of safe securities, while equity risk is distributed to equity investors. The convertible arbitrage strategy is constrained, both in practice and in our theory, because the hedge funds realizing the arbitrage have a limited ability to issue the securities replicating the convertible bond. As a result, convertible bond cheapness increases when arbitrageurs have greater difficulties issuing liabilities, such as during the 1998 LTCM crisis, the 2005 convertible arbitrage meltdown, and the 2008 credit crisis. 
Concave beta pricing. Denote tree return as $R_{t+1}\left(\delta \mid s^{t+1}\right) \equiv \frac{\delta_{t+1}\left(s^{t+1}\right)+P_{t+1}\left(\delta \mid s^{t+1}\right)}{P_{t}\left(\delta \mid s^{t}\right)}$ and consider any $K$ factors with expected returns normalized to zero. By linear projection onto the factors, we can write the tree returns as

$$
R_{t+1}\left(\delta \mid s^{t+1}\right)=\mathbb{E}_{t}\left[R_{t+1}\left(\delta \mid s^{t+1}\right)\right]+\sum_{k=1}^{K} \beta_{k}\left(\delta \mid s^{t}\right) F_{k, t+1}\left(s^{t+1}\right)+\epsilon_{t+1}\left(\delta \mid s^{t+1}\right),
$$

with

$$
0=\mathbb{E}_{t}\left[F_{k, t+1}\left(s^{t+1}\right)\right]=\mathbb{E}_{t}\left[\epsilon_{t+1}\left(\delta \mid s^{t+1}\right)\right]=\mathbb{E}_{t}\left[F_{k, t+1}\left(s^{t+1}\right) \epsilon_{t+1}\left(\delta \mid s^{t+1}\right)\right] .
$$

The next proposition states that if the factors orthogonalize tree returns with respect to all agents' private valuation operators (i.e., agents' stochastic discount factors), then a tree expected return is concave in the factor exposures.

Proposition 5 Let $M_{i t+1}\left(s^{t+1}\right) \equiv Q_{i t+1}\left(s^{t+1}\right) / \pi_{t+1}\left(s_{t+1} \mid s^{t}\right)$ denote the stochastic discount factor for agent i. If

$$
\mathbb{E}_{t}\left[M_{i t+1}\left(s^{t+1}\right) \epsilon_{t+1}\left(\delta \mid s^{t+1}\right)\right]=0 \quad \text { for all } \delta \text { and } i,
$$

then the expected return, $\mathbb{E}_{t}\left[R_{t+1}\left(\delta \mid s^{t+1}\right)\right]$, is a concave function of factor exposures $\left(\beta_{k}\left(\delta \mid s^{t}\right)\right)_{k=1}^{K}$.

Condition (29) holds in particular if the factors correspond to the stochastic discount factors of the different agents. The intuition for the concavity of expected returns in betas is similar to the intuition for the result that a tree is priced below a replicating portfolio of long positions in other trees (Proposition 4). Consider a tree with a vector $\beta\left(\delta \mid s^{t}\right)$ of factor exposures. That tree can be replicated, up to unpriced risk $\epsilon_{t+1}\left(\delta \mid s^{t+1}\right)$, with a portfolio of long positions in other trees such that a linear combination of their factor exposures are equal to $\beta\left(\delta \mid s^{t}\right)$. In line with Proposition 4, Proposition 5 states that the replicated tree has lower price and higher expected return than the replicating portfolio. Our theoretical result that expected returns are concave in factor betas is in the spirit of empirical evidence of concavity of the security market line (Frazzini and Pedersen, 2014; Hong and Sraer, 2016). 


\subsection{Optimal payoff sets vs. optimal tree sets}

In the analysis above, we have studied segmentation and asset pricing in terms of one-period ahead payoffs. However, these payoffs are in general endogenous since they depend on next-period prices. We now show that our analysis applies to the set of exogenous dividend streams instead of endogenous one-period-ahead payoffs, and discuss new insights.

When viewed as a Bellman equation, the recursive pricing formula (25) reveals that tree prices solve an intertemporal optimization problem: find the state-contingent sequence of asset holders with the highest valuation for the tree. More formally, define a sequence of agents as $J=\left\{j_{t}\left(s^{t}\right) \in\{1, \ldots, I\}: 0 \leq t<T, s^{t} \in S^{t}\right\}$, and let $\mathcal{J}$ denotes the set of all such sequences. Any sequence $J$ generates a private-valuation for node $\left(t, s^{t}\right)$ consumption defined recursively as

$$
q_{t+1}\left(s^{t+1} \mid J\right)=q_{t}\left(s^{t} \mid J\right) \times Q_{j_{t}\left(s^{t}\right), t+1}\left(s_{t+1} \mid s^{t}\right)
$$

with the convention that $q_{0}\left(s^{0} \mid J\right)=0$ for all $J$. Then, a standard optimality verification argument (in Online Appendix X.1) shows that:

$$
P_{t}\left(\delta \mid s^{t}\right)=\max _{J \in \mathcal{J}} \sum_{\left(u, s^{u}\right) \succ\left(t, s^{t}\right)} \frac{q_{u}\left(s^{u} \mid J\right)}{q_{t}\left(s^{t} \mid J\right)} \delta_{u}\left(s^{u}\right) .
$$

This formula reveals that the pricing functional is the maximum of a family of linear functions of $\delta$, each corresponding to a particular sequence of agents, $J$. Hence our earlier characterization of optimal payoff sets carries over to trees, but with one key difference: instead of characterizing the optimal payoff set of an individual agent, $j$, the Optimal Transport problem now characterizes the optimal tree set of a sequence of agents. The optimal tree set of agent $j$ is the union of optimal tree sets over all sequences $J$ of agents starting with agent $j$. Hence, the optimal tree set of agent $j$ is not necessarily convex.

Now turning to pricing, one immediate implication of (30) is that the pricing functional is a piecewise linear and convex function of dividend streams $\delta$. Another implication, that has been highlighted in prior work on asset pricing with heterogenous beliefs (Harrison and Kreps, 1978; Scheinkman and Xiong, 2003), is that the functional prices the option to re-sell the tree to a different type of agent at a later date. Formally, 
the price of the tree has to be greater than the buy-and-hold value that would be derived by a constant sequence of agents, and strictly so if the optimum of (30) is not attained by a constant sequence.

\section{Conclusion}

This paper offers a dynamic general equilibrium analysis of risk sharing and asset pricing when collateral is imperfectly pledgeable. This yields a rich set of implications on asset holdings (endogenous segmentation) and asset pricing (basis, concavity of expected returns in factor loadings).

While our model is set in the context of a pure exchange economy, it would be interesting to extend the analysis to study investment in production technologies. In our theoretical framework, these technologies have a dual role: they expand production possibilities but, at the same time, they can be used as collateral and thus improve risk-sharing and consumption smoothing. One would expect that, in some cases, there would be a tradeoff between technological and collateral efficiency, breaking standard separation results in classical theories. That is, a technology that leads to a large increase in production efficiency may be a poor collateral, and vice versa. The tradeoff between collateral and technological efficiency may have implications for international trade and for the nexus between technological and financial development. 


\section{References}

Aliprantis, Charalambos D. and Kim C. Border, Infinite Dimensional Analysis: A Hitchhiker's Guide, 3rd edition ed., Springer-Verlag Berlin Heidelberg, 2006. 7, 36, 39, 42

Alvarez, Fernando and Urban J. Jermann, "Efficiency, Equilibrium, and Asset Pricing with Risk of Default," Econometrica, 2000, 68, 775-797. 3, 5, 11, 17

Andrade, Gregor and Steven N. Kaplan, "How Costly is Financial (Not Economic) Distress? Evidence from Highly Leveraged Transactions that Became Distressed," Journal of Finance, 1998, 53, 1443-1493. 2

Araújo, Aloísio, Felix Kubler, and Susan Schommer, "Regulating collateral-requirement when markets are incomplete," Journal of Economic Theory, 2010, 147, 450-476. 5

Arrow, Kenneth J. and Gérard Debreu, "Existence of an Equilibrium for a Competitive Economy," Econometrica, 1954, 22 (265-290). 3, 13

Aurenhammer, Franz, "A Criterion for the Affine Equivalence of Cell Complexes in $R^{d}$ and Convex Polyhedra in $R^{d+1}, "$ Discrete Computational Geometry, 1987, 2, 49-64. 21, 54

_ , "Power Diagrams: Properties, Algorthms, and Applications," SIAM Journal on Computing, 1987, 16 (1), 78-96. 21, 54

Brumm, Johannes, Michael Grill, Felix Kubler, and Karl Schmedders, "Collateral Requirements and Asset Prices," International Economic Review, 2015, 56, 1-25. 5

Campbell, John Y., Stefano Giglio, and Parag Pathak, "Forced Sales and House Prices," American Economic Review, 2011, 101, 2108-2131. 2

Catherine, Sylvain, Paolo Sodini, and Yapei Zhang, "Countercyclical Income Risk and Portfolio Choice: Evidence from Sweden," 2020. Working paper University of Pennsylvania. 3

Chien, Yili and Hanno Lustig, "The Market Price of Aggregate Risk and the Wealth Distribution," Review of Financial Studies, 2009, 23, 1596-1650. 5, 9, 11, 12

Edmond, Chris and Simon Mongey, "Unbundling Labor," 2020. Working paper, University of Melbourne and University of Chicago. 6

Fleming, Michael J. and Asani Sarkar, "The Failure Resolution of Lehman Brothers," FRBNY Economic Policy Review, 2014, December, 175-206. 2

Fostel, Ana and John Geanakoplos, "Leverage Cycles and the Anxious Economy," American Economic Review, 2008, 98, 1211-44. 5, 6 
Frazzini, Andrea and Lasse Hejee Pedersen, "Betting agaisnt beta," Journal of Financial Economics, 2014, 111, 1-25. 4, 26

Galichon, Alfred, Optimal Transport Methods in Economics, Princeton University Press, 2016. 3, 19

Gârleanu, Nicolae and Lasse Heje Pedersen, "Margin-based asset pricing and deviations from the law of one price," Review of Financial Studies, 2011, 24 (6), 1980-2022. 5

Geanakoplos, John and William R. Zame, "Collateral Equilibrium, I: a Basic Framework," Economic Theory, $2014,56,443-492.5,6$

Geerolf, Francois, "Leverage and Disagreement," 2015. Working paper UCLA. 5

Gottardi, Piero and Felix Kubler, "Dynamic Competitive Economies with Complete Markets and Collateral Constraints," Review of Economic Studies, 2015, 82, 1119-1153. 5, 11, 12

Gromb, Denis and Dimitri Vayanos, "Equilibrium and welfare in markets with financially constrained arbitrageurs," Journal of financial Economics, 2002, 66 (2), 361-407. 5

_ and _, "The Dynamics of Financially Constrained Arbitrage," Journal of Finance, 2017, Forthcoming. 5

Harrison, J. Michael and David M. Kreps, "Speculative Behavior in a Stock Market with Heterogenous Expectations," Quarterly Journal of Economics, 1978, 92 (323-336). 27

Heckman, James and José Scheinkman, "The Importance of Bundling in a Gorman-Lancaster Model of Earnings," Review of Economic Studies, 1987, 54, 243-255. 6

Hindy, Ayman and Ming Huang, "Asset Pricing with Linear Collateral Constraints," Technical Report, Stanford University 1995. 5

Hong, Harrison and David Sraer, "Speculative Beta," Journal of Finance, 2016, Forthcoming. 4, 26

Jacquet, Nicolas L., "Asset Classes," Technical Report, Singapore Management University 2015. 6

Kehoe, Timothy J. and David K. Levine, "Debt-Constrained Asset Markets," Review of Economic Studies, 1993, 60, 865-888. 5

Kiyotaki, Nobuhiro and John Moore, "Credit Cycles," Journal of Political Economy, 1997, 105, 211-248. 2

Krusell, Per, Toshihiko Mukoyama, and Anthony A. Smith, "Asset prices in a Huggett Economy," Journal of Economic Theory, 2011, 146, 812-844. 21

Lagos, Ricardo, "Asset prices and liquidity in an exchange economy," Journal of Monetary Economics, 2010, 57, 913 - 930. 6 
Lenel, Moritz, "Safe Assets, Collateralized Lending and Monetary Policy," 2017. Working paper, Stanford University. 5

Lester, Benjamin, Andrew Postlewaite, and Randall Wright, "Information, Liquidity, Asset Prices, and Monetary Policy," Review of Economic Studies, 2012, 79, 1208-1238. 6

Li, Yiting Li, Guillaume Rocheteau, and Pierre-Olivier Weill, "Liquidity and the Threat of Fraudulent Assets," Journal of Political Economy, 2012, 120, 815-846. 6

Ljungqvist, Lars and Thomas J. Sargent, Recursive Macroeconomic Theory, third edition ed., Boston: MIT Press, 2012. 11

Luenberger, David, Optimization by vector space methods, John Wiley \& Son, 1969. 34, 46

Lustig, Hanno and Stijn Van Nieuwerburgh, "How much does household collateral constrain regional risk sharing?," Review of Economic Dynamics, 2010, 13 (2), 265-294. 5

Mas-Colell, Andreu, Michael D. Whinston, and Jerry R. Green, Microeconomic Theory, Oxford: Oxford University Press, 1995. 48

Mitchell, Mark and Todd Pulvino, "Arbitrage crashes and the speed of capital," Journal of Financial Economics, 2012, $104(3), 469-490.4,25$

Negishi, Takashi, "Welfare Economics and Existence of An Equilibrium for a Competitive Economy," Metroeconomica, 1960, 12, 92-97. 3, 13

Rampini, Adriano and S. Viswanathan, "Collateral, Risk Management, and the Distribution of Debt Capacity," Journal of Finance, 2010, 65, 2293-2322. 5, 10, 57, 59, 60

Rosen, Sherwin, "A Note on Aggregation of Skills and Labor Quality," The Journal of Human Resources, 1983, $18,425-431.6$

Scheinkman, José and Wei Xiong, "Overconfidence and Speculative Bubbles," Journal of Political Economy, 2003, 111, 1183-1219. 27

Stokey, Nancy L. and Robert E. Lucas, Recursive Methods in Economic Dynamics, Harvard University Press, 1989. $32,33,38,48$

Varadarajan, V.S., "Weak Convergence of Measures on Separable Metric Space," The Indian Journal of Statistics, $1958,19,15-22.32$

Venkateswaran, Venky and Randall Wright, "Pledgability and Liquidity: A New Monetarist Model of Financial and Macroeconomic Activity," 2013. 6

Ziegler, Günter M., Lectures on Polytopes, Springer, 1995. 21 


\section{Appendix}

We establish several of our results in the extended environment with a pledgeability parameter that is agent and tree specific. That is, we assume that each agent $i$ can pledge a fraction $\theta_{i}(\delta)$ of the next-period payoff of tree $\delta \in \Delta$, where $\theta_{i}(\delta) \in(0,1)$ for all $i$ and $\delta$, is continuous and uniformly bounded away from zero and one across all agents and trees.

In what follows we will use the following notations and definitions. For each $\left(t, s^{t}\right)$, we let $\Delta_{t}^{+}\left(s^{t}\right)$ denote the set of trees with non-zero continuation dividend, i.e. such that $\delta_{u}\left(s^{u}\right)>0$ for some $\left(u, s^{u}\right) \succ\left(t, s^{t}\right)$. The set of functions continuous on $\Delta$ is endowed with the metric induced by the sup norm. The set of positive and finite measures over $\Delta$ is denoted by $\mathcal{M}_{+}$and is endowed with the topology of weak convergence. ${ }^{20}$

Finally, as is standard, it is easier to establish equilibrium existence when prices are deflated to time zero. Namely, after fixing the price of consumption a time zero to be $q_{0}\left(s^{0}\right)$, we let the deflated or time-zero price of consumption at node $\left(t, s^{t}\right)$ to be $q_{t}\left(s^{t}\right) \equiv q_{0}\left(s^{0}\right) Q_{1}\left(s^{0}, s_{1}\right) Q_{2}\left(s^{1}, s_{2}\right) \ldots Q_{t}\left(s^{t-1}, s_{t}\right)$. Likewise, the deflated price of trees is given by the deflated functional $p_{t}\left(\delta \mid s^{t}\right) \equiv q_{t}\left(s^{t}\right) P_{t}\left(\delta \mid s^{t}\right)$. For the remainder of this appendix, we work with this deflated price system $(p, q)$. Correspondingly, we write the sequential budget constraint as:

$$
\begin{aligned}
& q_{t}\left(s^{t}\right) c_{i t}\left(s^{t}\right)+\int p_{t}\left(\delta \mid s^{t}\right) d N_{i t}\left(\delta \mid s^{t}\right)+\sum_{s} q_{t+1}\left(s^{t}, s\right) W_{i t+1}\left(s^{t}, s\right) \\
= & q_{t}\left(s^{t}\right) W_{i t}\left(s^{t}\right)+\sum_{s} q_{t+1}\left(s^{t}, s\right) e_{i t+1}\left(s^{t}, s\right)+\sum_{s} \int\left[q_{t+1}\left(s^{t}, s\right) \delta_{t}\left(s^{t}, s\right)+p_{t+1}\left(\delta \mid s^{t}, s\right)\right] d N_{i t}\left(\delta \mid s^{t}, s\right),
\end{aligned}
$$

for all $\left(t, s^{t}\right)$, and with the convention that time $T+1$ variables and time $T$ tree prices are equal to zero. Likewise, we write the incentive constraint as:

$$
q_{t+1}\left(s^{t}, s\right) W_{i t+1}\left(s^{t}, s\right) \geq q_{t+1}\left(s^{t}\right) e_{i t+1}\left(s^{t}, s\right)+\theta \int\left[q_{t+1}\left(s^{t}, s\right) \delta_{t+1}\left(s^{t}, s\right)+p_{t+1}\left(\delta \mid s^{t}, s\right)\right] d N_{i t}\left(\delta \mid s^{t}\right)
$$

\section{A Preliminary results}

\section{A.1 Normalized no-arbitrage price systems}

We let NA denote the set of normalized no-arbitrage price systems, that is, the set of $(p, q)$ such that consumption prices $q$ lie in the simplex:

$$
\sum_{\left(t, s^{t}\right)} q_{t}\left(s^{t}\right)=1,
$$

\footnotetext{
${ }^{20}$ Recall that a sequence $N^{\ell} \in \mathcal{M}_{+}$converges weakly towards some $N \in \mathcal{M}_{+}$if $\int f(\delta) d N^{\ell}(\delta) \rightarrow \int f(\delta) d N(\delta)$ for all functions $f$ continuous on $\Delta$. We use sequences instead of nets to define weak convergence because $\mathcal{M}_{+}$is metrizable (Varadarajan, 1958). The same sequential characterization of weak convergence is used by Stokey and Lucas (1989).
} 
tree prices $p_{t}\left(\delta \mid s^{t}\right)$ are continuous in $\delta \in \Delta$ for all $\left(t, s^{t}\right)$, and the no-arbitrage conditions hold:

$$
\begin{aligned}
q_{t}\left(s^{t}\right) & >0 \\
p_{t}\left(\delta \mid s^{t}\right) & \leq \sum_{s}\left[q_{t+1}\left(s^{t}, s\right) \delta_{t+1}\left(s^{t}, s\right)+p_{t+1}\left(\delta \mid s^{t}, s\right)\right] \\
p_{t}\left(\delta \mid s^{t}\right) & \geq\left(1-\theta_{i}(\delta)\right) \sum_{s}\left[q_{t+1}\left(s^{t}, s\right) \delta_{t+1}\left(s^{t}, s\right)+p_{t+1}\left(\delta \mid s^{t}, s\right)\right],
\end{aligned}
$$

where (35) must hold for all $\delta$, and (36) must hold for all agents $i$ and trees $\delta$, with a strict inequality if the continuation dividend is non-zero, $\delta \in \Delta_{t}^{+}\left(s^{t}\right)$.

Notice that we restrict attention here to price systems such that (35) holds for all $\delta \in \Delta$. Although this is stronger than the necessary condition for no-arbitrage of Lemma 1, this will not create problems for establishing existence. As will become clear, in any equilibrium, there always exists a price system such that (35) holds for all $\delta \in \Delta$. A useful property to keep in mind, shown in online Appendix I, is:

Lemma A.1 The closure of $\mathbb{N A}$, denoted by $\overline{\mathbb{N A}}$, is the set of price systems $(p, q)$ such that (34)-(36) hold with weak inequalities.

\section{A.2 Properties of the constraint correspondence}

The constraint correspondence is the set-valued function $\Gamma_{i}\left(W_{0}, p, q\right)$ mapping any initial cash-on-hand $W_{0} \in \mathbb{R}_{+}$and price system $(p, q) \in \overline{\mathbb{N A}}$ to the set of plans $\left(c_{i}, N_{i}\right)$ that are budget feasible and incentive compatible given the initial wealth $W_{0}$ and price system $(p, q)$. An important property for what follows is:

Proposition A.1 The constraint correspondence is non-empty, convex-valued, has a closed-graph over $\mathbb{R}_{+} \times \overline{\mathbb{N A}}$, and is lower hemi-continuous over $\mathbb{R}_{+} \times \mathbb{N A}$.

The challenging part of the proof, shown in online Appendix II, is to establish lower hemi-continuity. To understand why, recall that $\Gamma_{i}\left(W_{0}, p, q\right)$ is lower hemi-continuous at $\left(W_{0}, p, q\right)$ if for all $\left(W_{0}^{\ell}, p^{\ell}, q^{\ell}\right) \rightarrow\left(W_{0}, p, q\right)$, and for all $(c, N) \in \Gamma_{i}\left(W_{0}, p, q\right)$, there exists a sequence $\left(c^{\ell}, N^{\ell}\right) \rightarrow(c, N)$ such that, for all $\ell$ large enough, $\left(c^{\ell}, N^{\ell}\right) \in \Gamma_{i}\left(W_{0}^{\ell}, p^{\ell}, q^{\ell}\right)$ (see Section 3.3. in Stokey and Lucas, 1989). Without incentive constraints, the proof of lower hemi-continiuity is easy. Indeed, if $c \neq 0$, then for $\left(p^{\ell}, q^{\ell}\right) \rightarrow(p, q)$, one can finance some $\left(c^{\ell}, N^{\ell}\right) \in \Gamma_{i}\left(W_{0}^{\ell}, p^{\ell}, q^{\ell}\right)$ which is arbitrarily close to $(c, N)$ by reducing consumption slightly in some state. But this no longer works with our incentive constraints: indeed reducing consumption in some state corresponds to a reduction in the amounts of cash-on-hand planed for this state, which tightens incentive constraints.

Next, we define, for any $(p, q) \in \mathbb{N} A$, the demand correspondence of agent $i: Z_{i}(p, q)=\arg \max U_{i}\left(c_{i}\right)$ with respect to $\left(c_{i}, N_{i}\right) \in \Gamma_{i}\left(W_{0}, p, q\right)$, where $W_{0}$ is defined by (9). An important step in the classical proof of existence is to show that the excess demand becomes arbitrarily large when the price system nearly violates the no-arbitrage conditions.

Proposition A.2 Take any $(p, q) \in \overline{\mathbb{N A}} \backslash \mathbb{N A}$. Then there exists some $i$ such that, for any sequence $\left(p^{\ell}, q^{\ell}\right)$ in $\mathbb{N A}$ converging to $(p, q)$, any sequence of optimal demand $\left(c_{i}^{\ell}, N_{i}^{\ell}\right) \in Z_{i}\left(p^{\ell}, q^{\ell}\right)$ is unbounded in consumption.

The intuition is that, by our maintained assumptions and the no-abitrage conditions, agents initial tree wealth must be bounded away from zero at all $(p, q) \in \overline{\mathbb{N A}} \backslash \mathbb{N} A$. This allows the agents who are best able to pledge (with low $\left.\theta_{i}(\delta)\right)$ to take advantage of near arbitrage opportunities as $\left(p^{\ell}, q^{\ell}\right) \rightarrow(p, q)$ and guarantee unbounded consumption. The detailed proof is in Online Appendix III. 


\section{A.3 First-order necessary and sufficient conditions}

We state first-order conditions when agents maximize taking as given the deflated price system $(p, q)$, and subject to the corresponding budget and incentive constraints, (31) and (32). Without loss, we state the sequential budget constraints with weak inequalities. This allows us to apply Theorem 1 of chapter 8.3 and 8.4 in Luenberger (1969). In particular, since there are only a finite number of parametric constraints, the interior-point condition for the positive cone of the range of the parametric constraints is immediately satisfied. We thus obtain:

Proposition A.3 Let $(p, q)$ be a price system satisfying the no-arbitrage conditions of Lemma 1 . Then the plan $\left(c_{i}, N_{i}\right)$ solves agent $i$ 's problem if and only if it is budget and incentive feasible and there exists positive multipliers $\hat{\lambda}=\left\{\hat{\lambda}_{i t}\left(s^{t}\right), t \geq 0, s^{t} \in S^{t}\right\}$ and $\hat{\mu}=\left\{\hat{\mu}_{i t}\left(s^{t}\right), t \geq 1, s^{t} \in S^{t}\right\}$ such that:

$$
\begin{aligned}
& \beta^{t} \pi_{t}\left(s^{t}\right) u_{i}^{\prime}\left(c_{i t}\left(s^{t}\right)\right) \leq \hat{\lambda}_{i t}\left(s^{t}\right) q_{t}\left(s^{t}\right) \text { with " }=" \text { if } c_{i t}\left(s^{t}\right)>0 \\
& \hat{\lambda}_{i t}\left(s^{t}\right)=\hat{\lambda}_{i t+1}\left(s^{t+1}\right)+\hat{\mu}_{i t+1}\left(s^{t+1}\right) \\
& \hat{\mu}_{i t}\left(s^{t}\right)\left\{q_{t}\left(s^{t}\right) W_{i t}\left(s^{t}\right)-q_{t}\left(s^{t}\right) e_{i t}\left(s^{t}\right)-\int \theta_{i}(\delta)\left[q_{t}\left(s^{t}\right) \delta_{t}\left(s^{t}\right)+p_{t}\left(\delta \mid s^{t}\right)\right] d N_{i t-1}\left(s^{t}\right)\right\}=0 \\
& \int\left[\hat{v}_{i t}\left(\delta \mid s^{t}\right)-p_{t}\left(\delta \mid s^{t}\right)\right] d M(\delta) \leq 0 \text { for all } M \in \mathcal{M}_{+} \text {with " }=" \text { if } M=N_{i t}\left(s^{t}\right),
\end{aligned}
$$

where, for all $t<T$ :

$$
\hat{v}_{i t}\left(\delta \mid s^{t}\right) \equiv \sum_{s}\left(1-\frac{\theta_{i}(\delta) \hat{\mu}_{i t+1}\left(s^{t}, s\right)}{\hat{\lambda}_{i t}\left(s^{t}\right)}\right)\left[q_{t+1}\left(s^{t}, s\right) \delta_{t+1}\left(s^{t}, s\right)+p_{t+1}\left(\delta \mid s^{t}, s\right)\right] .
$$

Online appendix IV provides the proof and derives the corresponding first-order conditions for the non-deflated price system $(P, Q)$ shown in the text.

\section{A.4 Walras Law}

The classical proof of existence adds to the economy a fictitious player who sets price in order to maximize the value of excess demand - increasing the price of goods in positive excess demand and vice versa. In order to make an educated guess about the specification of the price player's objective in our setting, we now derive Walras Law that is, we calculate the value of the excess demand when all agents satisfy their budget constraints. The proof is in Online Appendix V.

Proposition A.4 Suppose an allocation $(c, N)_{i \in I}$ is budget feasible for all $i$ given some no-arbitrage price system $(p, q)$. Then:

$$
\sum_{\left(t, s^{t}\right)} q_{t}\left(s^{t}\right)\left(\sum_{i} c_{i t}\left(s^{t}\right)-\omega_{t}\left(s^{t}\right)\right)-\sum_{\left(t, s^{t}\right), t<T} \int b_{t}\left(\delta \mid s^{t}\right)\left(\sum_{i} d N_{i t}\left(\delta \mid s^{t}\right)-d \bar{N}(\delta)\right)=0
$$

where $\omega_{t}\left(s^{t}\right) \equiv \sum_{i} e_{i t}\left(s^{t}\right)+\int \delta_{t}\left(s^{t}\right) d \bar{N}(\delta)$ is the aggregate endowment and $b_{t}\left(\delta \mid s^{t}\right)$ is the basis functional generated by the price system $(p, q)$ at node $\left(t, s^{t}\right), t<T$ :

$$
b_{t}\left(\delta \mid s^{t}\right)=\sum_{s^{\prime}}\left(q_{t+1}\left(s^{t}, s\right) \delta_{t+1}\left(s^{t}, s\right)+p_{t+1}\left(\delta \mid s^{t}, s\right)\right)-p_{t}\left(s^{t}\right)
$$


with the convention that $p_{T}\left(s^{T}\right)=0$.

Without incentive constraints, there are no bases and the second term is equal to zero, because all that matter in agents intertemporal constraint is the present value of the dividend generated by the tree. In that case, Walras Law takes its standard form. In our setting with incentive constraints, Walras Law takes a non standard form for two reasons, giving rise to the second term in our expression of Walras Law. First, agents generate extra revenue with basis trades: purchases of trees financed by sales of replicating portfolios of Arrow securities. Second the bases depress the value of agents' initial tree endowment.

\section{B Proof of Theorem 1}

\section{B.1 Existence of equilibrium with finitely many trees}

We first provide an existence proof when the tree supply has finite support, and when agents can only choose portfolios in that support. That is, we replace $\Delta$ by some finite subset, $\left\{\delta_{1}, \ldots, \delta_{K}\right\} \subseteq \Delta$, and we choose a supply vector $\bar{N} \in \mathbb{R}_{+}^{K}$ such that the aggregate dividend is strictly positive in all states. Notice that, in this market setting, agents are restricted to choose portfolios in the finite subset $\left\{\delta_{1}, \ldots, \delta_{K}\right\}$ instead of in $\Delta$. Hence, portfolios are no longer finite measures but simply vectors, specifying the share holding of each tree $k$. Correspondingly we use the notation $n_{i t}\left(s^{t}\right) \in \mathbb{R}_{+}^{K}$ to denote the portfolio chosen by agent $i$ at node $\left(t, s^{t}\right)$. Likewise, asset prices at node $\left(t, s^{t}\right)$ are no longer represented by a function but by a vector $p_{t}\left(s^{t}\right) \in \mathbb{R}_{+}^{K}$. Finally, with some abuse of notation, $\delta_{t}\left(s^{t}\right)=\left(\delta_{1 t}\left(s^{t}\right), \delta_{2 t}\left(s^{t}\right), \ldots, \delta_{K t}\left(s^{t}\right)\right) \in \mathbb{R}_{+}^{K}$ now denote the vector of all tree dividends at node $\left(t, s^{t}\right)$.

\section{B.1.1 The fixed-point problem}

We now adapt the classical fixed-point problem to our setting. First, we let $B$ denote the set of $(c, n)$ such that

$$
\begin{aligned}
& 0 \leq c_{t}\left(s^{t}\right) \leq 2 \sum_{\left(u, s^{u}\right)} \omega_{u}\left(s^{u}\right) \\
& 0 \leq n_{t}\left(s^{t}\right) \leq 2 \bar{N}
\end{aligned}
$$

where $\omega_{t}\left(s^{t}\right)$ is the aggregate endowment, as defined in Proposition A.4. We let $Z_{i}^{B}(p, q)$ denote the demand correspondence of agent $i$ when she is artificially constrained to choose $\left(c_{i}, n_{i}\right) \in \Gamma_{i}\left(W_{0}, p, q\right) \cap B$, with $W_{0}$ defined according to (9). This correspondence now has compact values. It continues to have a closed graph. We verify in Online Appendix V.1 that it also continues to be lower hemi-continuous.

For any $0<\varepsilon<\min _{i, k} \theta_{i k}$, we let $\mathbb{N A}^{\varepsilon}$ denote the set of $(p, q) \in \mathbb{N} A$ satisfying :

$$
\begin{aligned}
& q_{t}\left(s^{t}\right) \geq \varepsilon \\
& p_{k t}\left(s^{t}\right) \geq\left(1-\theta_{i k}+\varepsilon\right) \sum_{s}\left(q_{t+1}\left(s^{t}, s\right) \delta_{k t+1}\left(s^{t}, s^{\prime}\right)+p_{k t+1}\left(s^{t}, s^{\prime}\right)\right) .
\end{aligned}
$$

for all $\left(t, s^{t}\right)$, all trees $k$, and all agents $i$. Note that $N A^{\varepsilon}$ is non empty since it contains price systems with zero basis. We use Walras Law shown in Proposition A.4 to formulate the objective of the price player. Given any profile of consumption and tree portfolio choices $\left(c_{i}, n_{i}\right)_{i \in I}$, we let the price player's problem be $Z_{0}^{\varepsilon}(c, n)=\arg \max q$. $\left(\sum_{i} c_{i}-\omega\right)-\mathfrak{b}(p, q) \cdot\left(\sum_{i} n_{i}-\bar{N}\right)$, with respect to $(p, q) \in \mathbb{N} A^{\varepsilon}$ and where $\mathfrak{b}(p, q)$ is the vector of bases generated 
by $(p, q): \mathfrak{b}_{t}\left(s^{t} \mid p, q\right) \equiv \sum_{s}\left(q_{t+1}\left(s^{t}, s\right) \delta_{t+1}\left(s^{t}, s\right)+p_{t+1}\left(s^{t}, s\right)\right)-p_{t}\left(s^{t}\right)$. for each $\left(t, s^{t}\right), t<T$, with the convention that $p_{T}\left(s^{T}\right)=0$. Next, we define the correspondence $\Psi: \mathbb{N A}^{\varepsilon} \times B^{I} \rightarrow \mathbb{N} A^{\varepsilon} \times B^{I}$ by

$$
\Psi^{\varepsilon}\left(p, q,\left(c_{i}, n_{i}\right)_{i \in I}\right)=Z_{0}^{\varepsilon}(c, n) \times Z_{1}^{B}(p, q) \ldots \times Z_{I}^{B}(p, q) .
$$

We show in Appendix V.2 that:

Proposition B.1 The correpondence $\Psi^{\varepsilon}$ has a fixed point which, for $\varepsilon$ small enough, is the basis of an equilibrium.

The argument is very similar to the one of the classical proof. The key difference is that there are bases, which create a second term in the price player's objective, that the price player can only manipulate by choosing prices respecting no-arbitrage conditions. Hence, relative to the classical proof, the price player faces different objective and constraints, which makes it more difficult to establish that all markets clear.

\section{B.2 Existence of equilibrium when the supply has a finite support}

The case of a finite support is almost identical to the case of finitely many trees. The only difference is that agents have a larger choice set: instead of being restricted to hold trees in $\left\{\delta_{1}, \delta_{2}, \ldots, \delta_{K}\right\}$, they can choose to hold any tree in $\Delta$ : formally, instead of choosing vectors $n \in \mathbb{R}_{+}^{K}$ representing their holdings of each tree in the support $\left\{\delta_{1}, \delta_{2}, \ldots, \delta_{K}\right\}$, they now choose positive finite measures $N \in \mathcal{M}_{+}$representing their holdings of each tree in $\Delta$. Correspondingly, the equilibrium must price all trees in $\Delta$, and not only the trees in the support $\left\{\delta_{1}, \delta_{2}, \ldots, \delta_{K}\right\}$. The following Lemma, proved in Appendix V.3, establishes that it is possible to choose a continuous price functional, defined over $\Delta$, such that the equilibrium identified in the previous section remains unchanged when agents can choose to hold any tree in $\Delta$.

Lemma B.1 Consider any equilibrium price system $(p, q)$ and allocation $(c, n)$ with a finite number of trees $\left\{\delta_{1}, \ldots, \delta_{K}\right\}$ in supply $\bar{N}=\left(n_{1}, \ldots, n_{K}\right)$. Then, there exists an extension $\hat{p}$ of the price vector $p$ to the set $\Delta$, such that

1. the function $\hat{p}$ belongs to an equi-continuous family $\mathcal{P}$;

2. the price system $(\hat{p}, q)$ belongs to $\mathrm{NA}$;

3. demands remain optimal when agents can choose to hold any tree in $\Delta$, given $(\hat{p}, q)$.

The price functional $\hat{p}$ is obtained by taking first-order conditions, using the formula given Proposition A.3, and has a simple intepretation: it is the maximum marginal valuation of future payoffs, across all agents. It is intuitive that it belongs to an equi-continuous family, since it can be written as a function that is jointly continuous with respect to the dividend stream and the intertemporal marginal rates of substitutions of finitely many agents across finitely many periods. See Appendix V.3 for the precise argument.

\section{B.3 Existence of equilibrium for an arbitrary supply}

Let $\bar{N}$ be an arbitrary positive finite measure over $\Delta$. By the density Theorem 15.10 in Aliprantis and Border (2006), easily extended to the case of finite measures, there exists a sequence of measures $\bar{N}^{\ell}$ with finite support converging weakly towards $\bar{N}$. Invoking Lemma B.1, for each $\ell$, there exists an equilibrium $\left(p^{\ell}, q^{\ell}, c^{\ell}, N^{\ell}\right)$. But this sequence of equilibria remains trapped in a compact set. ${ }^{21}$ This allows to extract a subsequence converging weakly to some $(p, q, c, N)$ (and keep the same notations).

\footnotetext{
${ }^{21}$ Indeed the $p^{\ell}$ belong to $\mathcal{P}$, an equi-continuous family of functions, the vector of consumption price $q^{\ell}$ is bounded by one, the vector of consumption $c^{\ell}$ is bounded by the aggregate endowment, and tree-market clearing ensures that each measures $N_{i}^{\ell}$ is bounded by $\bar{N}^{\ell}(\Delta) \leq 2 \bar{N}(\Delta)$ for all $\ell$ large enough which, together with Theorem 15.11 in Aliprantis and Border (2006), ensures weak compactness.
} 
By passing to the limit in the market-clearing conditions, one finds that the limiting consumption and portfolios are feasible. By passing to the limit in the budget and incentive constraints, one obtains that the limiting consumptions and portfolios belong to the constraint set of each agents: $\left(c_{i}, N_{i}\right) \in \Gamma_{i}\left(W_{i 0}, p, q\right)$ for each $i$, where $W_{i 0}$ is defined according to (9).

What remains to be shown is that the limiting consumptions and portfolios are optimal. To that end, we first note that the limiting price system $(p, q)$ must belong to $\mathbb{N A}$ : otherwise, if instead $(p, q) \in \overline{\mathbb{N} A} \backslash \mathbb{N} A$, then by Proposition A.2, easily extended to account for the fact that agents' tree endowment $\alpha_{i} \bar{N}^{\ell}$ varies along the sequence, the consumption of some agent would grow unbounded as $\ell \rightarrow \infty$, which would contradict market clearing. By Proposition A.1, it follows that the constraint correspondence of each agent is lower-hemi-continuous at $\left(W_{i 0}, p, q\right)$, where $W_{i 0}$ is defined according to (9). Now consider any agent $i$ and any $\left(\hat{c}_{i}, \hat{N}_{i}\right) \in \Gamma_{i}\left(W_{i 0}, p, q\right)$, where $W_{i 0}$ is defined according to (9). Lower-hemi-continuity ensures that there exists some sequence $\left(\hat{c}_{i}^{\ell}, \hat{N}_{i}^{\ell}\right) \in \Gamma_{i}\left(W_{i 0}^{\ell}, p^{\ell}, q^{\ell}\right)$, where $W_{i 0}^{\ell}$ is defined according to $(9)$, such that $\left(\hat{c}_{i}^{\ell}, \hat{N}_{i}^{\ell}\right)$ converges towards $(\hat{c}, \hat{N})$. Clearly, for each $\ell, U_{i}\left(c_{i}^{\ell}\right) \geq U_{i}\left(\hat{c}_{i}^{\ell}\right)$, where $c_{i}^{\ell}$ is the optimal consumption demand in the equilibrium given the equilibrium $\left(p^{\ell}, q^{\ell}\right)$ identified above. Passing to the limit, we obtain $U_{i}\left(c_{i}\right) \geq U_{i}\left(\hat{c}_{i}\right)$, which means that $\left(c_{i}, N_{i}\right)$ is indeed optimal given $(p, q)$.

\section{Proof of Corollary 1}

See Online Appendix VI.

\section{Proof of Proposition 1}

We start by deriving a necessary and sufficient condition for a complete market equilibrium to be IC implementable. The proof is in Online Appendix VII.

Lemma D.1 A complete market equilibrium $(q, c)$ is IC-implementable if and only if there exists a feasible tree allocation $N$ such that

$$
\sum_{\left(u, s^{u}\right) \succeq\left(t, s^{t}\right)} q_{u}\left(s^{u}\right)\left(c_{i u}\left(s^{u}\right)-e_{i u}\left(s^{u}\right)\right) \geq \theta \int\left[q_{t}\left(s^{t}\right) \delta_{t}\left(s^{t}\right)+p_{t}\left(\delta \mid s^{t}\right)\right] d N_{i t-1}\left(\delta \mid s^{t-1}\right),
$$

for all agents and at all nodes following time zero, that is for all $i$, all $\left(t, s^{t}\right), t \geq 1$.

Point 1. If Inada conditions are satisfied then, in a complete-market equilibrium the consumptions of all agents are bounded away from zero at all nodes. If in addition labor income is small enough, then the left-hand side of (47) is strictly positive. It then follows that (47) holds with the feasible tree allocation $N_{i}=\bar{N} / I$ and $\theta$ small enough.

Point 2. Consider a complete-market equilibrium $(c, q)$ when agents have heterogenous CRRA utility. Because of complete market, it follows that consumption of agent $i$ can be written as function of the aggregate endowment only, $c_{i t}\left(s^{t}\right)=y_{t}\left(s^{t}\right) f_{i}\left[y_{t}\left(s^{t}\right)\right]$, where $f_{i}$ denotes the consumption share. Because of heterogeneity in CRRA utility it follows that the consumption share function, $f_{i}$ is strictly increasing in $y$ for the least risk averse agent.

Now assume, towards a contradiction, that this equilibrium is implementable with just one tree for arbitrarily small labor income, and pledgeability parameter arbitrarily close to one. That is, there exists a sequence of labor endowment, $e^{p} \rightarrow 0$, of pledgeability parameter $\theta^{p} \rightarrow 1$ such that the complete market equilibrium is IC implementable for all $p$. Using (47) in the last period, this means that there exists some tree holdings $n_{i T-1}\left(s^{T-1}\right)$ such that:

$$
c_{i T}\left(s^{T}\right)-e_{i T}^{p}\left(s^{T}\right) \geq \theta^{p} n_{i T-1}^{p}\left(s^{T-1}\right) \delta_{T}^{p}\left(s^{T}\right),
$$


where $\delta_{T}^{p}\left(s^{T}\right)$ is the dividend of the tree and $\sum_{i} n_{i T}^{p}\left(s^{T}\right)=1$, (normalizing the aggregate supply of the tree to one). Dividing though by the aggregate endowment, this gives:

$$
f_{i}\left[y_{T}\left(s^{T}\right)\right] \geq \theta^{p} n_{i T-1}^{p}\left(s^{T-1}\right) \frac{\delta_{T}^{p}\left(s^{T}\right)}{y_{T}\left(s^{T}\right)}+\frac{e_{i T}^{p}\left(s^{T}\right)}{y_{T}\left(s^{T}\right)} .
$$

Given that $n_{i T-1}^{p}\left(s^{T-1}\right) \in[0,1]$, we can extract subsequences for each $i$ converging to $n_{i}^{\star}$ such that $\sum n_{i}^{\star}=1$. Moreover, since the labor endowment $e^{p} \rightarrow 0$, it follows that the tree dividend $\delta_{T}^{p}\left(s^{T}\right) \rightarrow y_{T}\left(s^{T}\right)$. With this in mind, when we go to the limit, we find $f_{i}\left[y_{T}\left(s^{T}\right)\right] \geq n_{i}^{\star}$, for all $i$. But since $\sum_{i} f_{i}\left[y_{T}\left(s^{T}\right)\right]=\sum_{i} n_{i}^{\star}=1$, it follows that $f_{i}\left[y_{T}\left(s^{T}\right)\right]=n_{i}^{\star}$ for all $i$, which is a contradiction since we noted earlier that the consumption-share function $f_{i}(y)$ is strictly increasing for the least risk-averse agent.

Point 3. Fix some strictly positive labor income $e \gg 0$ such that agents' intertemporal marginal rate of substitutions are not equalized when evaluated at $e_{i}$. Towards a contradiction, consider a sequence $\bar{N}^{\ell}$ such that $\bar{N}^{\ell}(\Delta) \rightarrow 0$, and a corresponding sequence of equilibria $\left(p^{\ell}, q^{\ell}, c^{\ell}, N^{\ell}\right)$ such that $\left(q^{\ell}, c^{\ell}\right)$ is a complete-market equilibrium for all $\ell$. Proposition VIII.2 in the Online Appendix shows that $\left(p^{\ell}, q^{\ell}, c^{\ell}, N^{\ell}\right)$ converges to the unique equilibrium that obtain when $\bar{N}(\Delta)=0$, what Proposition VIII.1 in the Online Appendix calls a "zero-collateral equilibrium". In particular $c_{i t}^{\ell}\left(s^{t}\right)$ converges to $e_{i t}\left(s^{t}\right)$. It follows that consumptions are strictly positive and intertemporal marginal rate of substitution are not equalized, implying that the equilibrium allocation cannot coincide with that of a complete-market equilibrium, a contradiction.

Point 4. Consider two distributions of tree supplies $\bar{N}^{\prime}$ and $\bar{N}$. Then, we say that $\bar{N}$ is less dispersed than $\bar{N}^{\prime}$ if there exists a transition probability function $d \Omega\left(\delta^{\prime} \mid \delta\right)$ (see Definition 8.1 in Stokey and Lucas, 1989) representing the weight of tree $\delta^{\prime}$ in a replicating portfolio for tree $\delta$, such that two conditions are satisfied. First, the portfolio $d \Omega\left(\delta^{\prime} \mid \delta\right)$ must replicate $\delta$ :

$$
\int_{\delta^{\prime}} \delta^{\prime} d \Omega\left(\delta^{\prime} \mid \delta\right)=\delta, \text { for all } \delta \in \Delta
$$

Second, the distribution of supplies must satisfy the consistency condition:

$$
\bar{N}^{\prime}(A)=\int_{\delta} \Omega(A \mid \delta) d \bar{N}(\delta)
$$

for all Borel sets $A$ of $\Delta$. This ensures that the measure of trees in the set $A, \bar{N}^{\prime}(A)$, is obtained by adding up the measures of trees in $A$ found in all replicating portfolios, $\Omega(A \mid \delta) d \bar{N}(\delta)$. One can directly verify that the two distributions, $\bar{N}$ and $\bar{N}^{\prime}$, have identical aggregate dividend:

$$
\int_{\delta^{\prime}} \delta^{\prime} d \bar{N}^{\prime}\left(\delta^{\prime}\right)=\int_{\delta^{\prime}} \int_{\delta} \delta^{\prime} d \Omega\left(\delta^{\prime} \mid \delta\right) d \bar{N}(\delta)=\int_{\delta} \delta d \bar{N}(\delta)
$$

where the first equality follows from (49) (or, more precisely, from Theorem 8.3 in Stokey and Lucas, 1989) and the second equality follows from (48).

The result then follows almost directly from the definition. Suppose that the complete market equilibrium under consideration is implemented for some $\theta$, given some distribution $\bar{N}$. Consider an increase in tree supply dispersion from $\bar{N}$ to $\bar{N}^{\prime}$. Then all agents can replicate their portfolio holdings under $\bar{N}$ by choosing tree portfolios $N_{i}^{\prime}(A)=\int_{\delta} \Omega(A \mid \delta) d N_{i}(\delta)$, where the $\left(t, s^{t}\right)$ notation is suppressed for simplicity. From equation (49), these tree portfolios satisfy market clearing. The sequential budget constraints and the incentive constraints of each agent continue to hold because, under complete market, tree prices are linear in $\delta$ and because of equation (48). This 
shows that agents' choice remain budget and incentive feasible, and continue to satisfy market clearing. Optimality follows because, by our maintained assumption of IC implementability, the agents' choice attain an upper bound on their maximum attainable utility: the utility they can attain under the same price system but without incentive constraints.

\section{E Proof of Proposition 3}

Let $V(\alpha, x) \equiv \beta g^{-\gamma}\left[\pi_{1}\left(1+k_{1} \alpha\right)^{\phi \gamma} x+\pi_{2}\left(1-k_{2} \alpha\right)^{\phi \gamma}(1-x)\right]$ denote the valuation functional for payoffs in the simplex, $(x, 1-x)$. Under the maintained assumption of the proposition, $\phi \gamma<1$, the function $\alpha \mapsto V(\alpha, x)$ is strictly concave in $\alpha$. Moreover, $\frac{\partial V}{\partial \alpha}=\phi \gamma\left(k_{1} \pi_{1}\left(1+k_{1} \alpha\right)^{\phi \gamma-1} x-k_{2} \pi_{2}\left(1-k_{2} \alpha\right)^{\phi \gamma-1}(1-x)\right)$ is positive at $\alpha=0$ if and only if

$$
x \geq x^{\star}(0)=\frac{k_{2} \pi_{2}}{k_{1} \pi_{1}+k_{2} \pi_{2}},
$$

and goes to minus infinity as $\alpha \rightarrow 1 / k_{2}$. It follows that all payoffs $x \leq x^{\star}$ belong to the optimal payoff set of $\alpha_{1}=0$. For payoffs $x>x^{\star}(0)$, there exists some $\alpha^{\star}(x)>0$ such that $V(x, \alpha)$ achieves a strict maximum at $\alpha=\alpha^{\star}(x)$. Given that $\partial^{2} V / \partial x \partial \alpha>0$, it follows that $\alpha^{\star}(x)$ is strictly increasing. One can also verify that $\alpha^{\star}(x)$ goes to $1 / k_{2}$ as $x \rightarrow 1$. Now considering the inverse function, it follows that, for for each $\alpha \in\left(0,1 / k_{2}\right]$, there exists some $x^{\star}(\alpha) \in\left(x^{\star}(0), 1\right]$ such that $\alpha$ has strictly higher valuation than any other agents for all payoffs near $x^{\star}(\alpha)$. Now since we have a finite number of agents $\alpha_{1}=0<\alpha_{2}<\ldots<\alpha_{I}$, it follows that, for all $i>2, \alpha_{i}$ values all payoffs near $x^{\star}\left(\alpha_{i}\right)$ the most. Together with our earlier observations that $X_{i}$ are intervals, increasing in $\alpha_{i}$, it follows that there is a strictly increasing sequence:

$$
x_{0}=0<x_{1}<x_{2}<\ldots<x_{I}=1
$$

such that agent $i$ holds all the payoffs in $X\left(\alpha_{i}\right)=\left[x_{i-1}, x_{i}\right]$, and $x_{i-1}<x^{\star}\left(\alpha_{i}\right)<x_{i}$.

\section{F Proof of Proposition 4}

Suppressing the $\left(t, s^{t}\right)$ notation for simplicity, let $\delta \mapsto x(\delta) \in \mathbb{R}_{+}^{S}$ denote the function that maps a tree $\delta$ into its vector of one-period ahead payoffs. Let $\hat{M}$ denote the measure on payoffs $x \in \mathbb{R}_{+}^{S}$, induced by the portfolio $M$ : that is, for all Borel set $B$ of $\mathbb{R}_{+}^{S}, \hat{M}(B)=M\left(x^{-1}(B)\right)$. With this notation, the replicating porftolio condition writes $x(\delta)=\int x^{\prime} d \hat{M}\left(x^{\prime}\right)$. Let $i$ be the type of some agent who is the best holder of tree $\delta$, that is $x(\delta) \in X_{i}$. With this notation, the maintained assumption of the proposition implies that $X_{i}$ does not contain (almost) all trees of the replicating portfolio, or $\hat{M}\left(\mathbb{R}_{+}^{S} \backslash X_{i}\right)>0$. The price of the replicating portfolio writes:

$$
\begin{aligned}
\int P\left(\delta^{\prime}\right) d M\left(\delta^{\prime}\right) & =\int \max _{j} Q_{j} \cdot x\left(\delta^{\prime}\right) d M\left(\delta^{\prime}\right)=\int \max _{j} Q_{j} \cdot x^{\prime} d \hat{M}\left(x^{\prime}\right) \\
& >\int Q_{i} \cdot x^{\prime} d \hat{M}\left(x^{\prime}\right)=P(\delta),
\end{aligned}
$$

where: the first equality on the first line follows by definition of the pricing functional for trees; the second equality on the first line line follows by the change of variable formula (see Theorem 13.46 in Aliprantis and Border, 2006); the strict inequality on the second line follows because $\max _{j} Q_{j} \cdot x^{\prime}>Q_{i} \cdot x^{\prime}$ for all $x^{\prime} \in \mathbb{R}_{+}^{S} \backslash X_{i}$ and $\hat{M}\left(\mathbb{R}_{+}^{S} \backslash X_{i}\right)>0$; the equality on the second line follows because the payoff of the portfolio $\hat{M}$ replicate the payoff of tree $\delta$, and because the price of tree $\delta$ is equal to the private valuation of agent $i$ for that tree. 


\section{G Proof of Proposition 5}

The proposition is an implication of the following Lemma:

Lemma G.1 Let $f(x, y)$ be some real-valued function of $(x, y) \in \mathbb{R}^{K} \times \mathbb{R}$. Assume that, $f(x, y)$ is convex in $(x, y)$, and strictly increasing in $y$. Suppose that, for each $x, f(x, y)=0$ has a solution, denoted by $\phi(x)$. Then, $\phi(x)$ is concave in $x$.

For a proof, note that the convexity of $f(x, y)$ with respect to $(x, y)$ implies:

$$
f\left(w_{1} x_{1}+w_{2} x_{2}, w_{1} \phi\left(x_{1}\right)+w_{2} \phi\left(x_{2}\right)\right) \leq w_{1} f\left(x_{1}, \phi\left(x_{1}\right)\right)+w_{2} f\left(x_{2}, \phi\left(x_{2}\right)\right)=0
$$

But since $f(x, y)$ is strictly increasing in $y, w_{1} \phi\left(x_{1}\right)+w_{2} \phi\left(x_{2}\right) \leq \phi\left(w_{1} x_{1}+w_{2} x_{2}\right)$.

Now let us turn to the proposition. Omitting the $\delta$ and $s^{t+1}$ arguments for notational simplicity, we first note that, in terms of returns and agent-specific stochastic discount factors, the the priciging formula (25) writes

$$
1=\max _{i} \mathbb{E}_{t}\left[M_{i t+1} R_{t+1}\right]
$$

Now using the factor decomposition of returns, (27), together with the assumed orthogonality condition $\mathbb{E}_{t}\left[M_{i t+1} \epsilon_{t+1}\right]=$ 0 , this can be written:

$$
\max _{i}\left\{\mathbb{E}_{t}\left[R_{t+1}\right] \mathbb{E}_{t}\left[M_{i t+1}\right]+\sum_{k=1}^{K} \beta_{k} \mathbb{E}_{t}\left[M_{i t+1} F_{k t+1}\right]-1\right\}=0 .
$$

The left-hand side can be written as $f(x, y)=0$, where $x$ is the vector of beta's, $\left(\beta_{k}\right)_{k=1}^{K}$, and $y$ is the expected return of the asset, $\mathbb{E}_{t}\left[R_{t+1}\right]$. The function $f(x, y)$ is convex, since it is the maximum of affine functions of $(x, y)$. It is strictly increasing in $y$, since the stochastic discount factors are strictly positive. We also have that $\lim _{y \rightarrow-\infty} f(x, y)=-\infty$ and $\lim _{y \rightarrow+\infty} f(x, y)=+\infty$, hence $f(x, y)=0$ has a unique solution. Now we apply Lemma G.1 and obtain that the solution $\phi(x)$ of the equation $f(x, y)=0$ is concave in $x$. 


\section{Online Appendix (not for publication)}

\section{Proof of Lemma A.1}

It is clear that $\overline{\mathrm{NA}}$ is included in the set defined by (34), (35), (36) with weak inequalities. For the reverse inclusion, consider any $(p, q)$ in the set defined by $(34),(35),(36)$ with weak inequalities, and any $\varepsilon>0$. Let

$$
q_{t}^{\varepsilon}\left(s^{t}\right)=q_{t}\left(s^{t}\right)+\varepsilon,
$$

and, working backward from time $T-1$ :

$$
p_{t}^{\varepsilon}\left(\delta s^{t}\right)=p_{t}\left(\delta \mid s^{t}\right)+\varepsilon \sum_{s} \delta_{t+1}\left(s^{t}, s\right)+\sum_{s}\left(p_{t+1}^{\varepsilon}\left(s^{t}, s\right)-p_{t+1}\left(s^{t}, s\right)\right)
$$

with the convention that $p_{T}\left(s^{T}\right)=p_{T}^{\varepsilon}\left(s^{T}\right)=0$. We prove by backward induction that $\left(p^{\varepsilon}, q^{\varepsilon}\right)$ satisfies the no-arbitrage conditions (34)-(35)-(35), as well as:

$$
p_{t}^{\varepsilon}\left(\delta \mid s^{t}\right) \geq p_{t}\left(\delta \mid s^{t}\right) \text { with }>\text { if } \delta \in \Delta_{t}^{+}\left(s^{t}\right) .
$$

First, notice that, (34) holds by construction at all $\left(t, s^{t}\right)$, while (34), (35), (36) and (54) hold by construction at time $T$. Now we show that they must hold at time $t$ if they hold at time $t+1 \leq T$.

- Evidently, since (54) holds at $t+1$, the definition of $p_{t}^{\varepsilon}\left(s^{t}\right)$ implies that holds with weak inequality at time $t$ for all $\delta$. It clearly holds with a strict inequality for all $\delta \in \Delta_{t}^{+}\left(s^{t}\right)$ such that $\delta_{t+1}\left(s^{t}, s\right)>0$ for some $s$. If $\delta \in \Delta_{t}^{+}\left(s^{t}\right)$ but $\delta_{t+1}\left(s^{t}, s\right)=0$ for all $s$, then $\delta \in \Delta_{t+1}^{+}\left(s^{t}, s\right)$ for some $s$, and the strict inequality follows from (53) together with our induction hypothesis that (54) holds at time $t+1$.

- Since (35) holds with a weak inequality for $(p, q)$ :

$$
\begin{gathered}
p_{t}^{\varepsilon}\left(\delta \mid s^{t}\right) \leq \sum_{s}\left[q_{t+1}\left(s^{t}, s\right) \delta_{t+1}\left(s^{t}, s\right)+p_{t+1}\left(\delta \mid s^{t}, s\right)\right]+\varepsilon \sum_{s} \delta_{t+1}\left(s^{t}, s\right) \\
\quad+\sum_{s}\left[p_{t+1}^{\varepsilon}\left(\delta \mid s^{t}, s\right)-p_{t+1}\left(\delta \mid s^{t}, s\right)\right] \\
=\sum_{s}\left[q_{t+1}^{\varepsilon}\left(s^{t}, s\right) \delta_{t+1}\left(s^{t}, s\right)+p_{t+1}^{\varepsilon}\left(\delta \mid s^{t}, s\right)\right]
\end{gathered}
$$

Hence (35) holds for $\left(p^{\varepsilon}, q^{\varepsilon}\right)$ at time $t$. 
- Likewise, since (36) holds with weak inequality for $(p, q)$ :

$$
\begin{aligned}
p_{t}^{\varepsilon}\left(\delta \mid s^{t}\right) \geq(1- & \left.\theta_{i}(\delta)\right) \sum_{s}\left[q_{t+1}\left(s^{t}, s\right) \delta_{t+1}\left(s^{t}, s\right)+p_{t+1}\left(\delta \mid s^{t}, s\right)\right]+\varepsilon \sum_{s} \delta_{t+1}\left(s^{t}, s\right) \\
& +\sum_{s}\left[p_{t+1}^{\varepsilon}\left(\delta \mid s^{t}, s\right)-p_{t+1}\left(\delta \mid s^{t}, s\right)\right] \\
= & \left(1-\theta_{i}(\delta)\right) \sum_{s}\left[q_{t+1}^{\varepsilon}\left(s^{t}, s\right) \delta_{t+1}\left(s^{t}, s\right)+p_{t+1}^{\varepsilon}\left(\delta \mid s^{t}, s\right)\right] \\
& +\theta_{i}(\delta)\left[p_{t}^{\varepsilon}\left(\delta \mid s^{t}\right)-p_{t}\left(\delta \mid s^{t}\right)\right] .
\end{aligned}
$$

Together with the fact, shown in the first bullet point, that (54) holds at time $\left(t, s^{t}\right)$, we obtain (36).

After scaling down $q^{\varepsilon}$ and $p^{\varepsilon}$ by the same constant so that the normalization (33) holds, we obtain a price system in NA. This price system clearly converges to $(p, q)$ as $\varepsilon \rightarrow 0$. Hence, $(p, q) \in \overline{\mathbb{N} A}$.

\section{Proof of Proposition A.1}

It is clear that $\Gamma_{i}\left(W_{0}, p, q\right)$ is non-empty because it contains the hand-to-mouth-plan, specifically $N_{i}=0, c_{i t}\left(s^{t}\right)=$ $W_{i t}\left(s^{t}\right)$ and, for $t>0, W_{i t}\left(s^{t}\right)=e_{i t}\left(s^{t}\right)$. The constraint correspondence is convex-valued because it is defined by a system of weak linear inequalities. The closed graph property is also immediate because the correspondence is defined by weak inequalities, all jointly continuous in $\left(W_{0}, p, q\right)$ and $\left(c_{i}, N_{i}\right){ }^{22}$ The only thing left to show is lower hemi-continuity.

\section{II.1 The one-period ahead constraint correspondence}

In this subsection, we fix some node $\left(t, s^{t}\right)$ and establish the lower hemi-continuity of the one-period ahead constraint correspondence. Since the node is fixed, we suppress explicit reference to it to simplify notations. Instead, we use the " 0 " subscript for variables indexed by $\left(t, s^{t}\right)$, and the " 1 " subscript, together with the argument $s$, for variables indexed by $\left(t, s^{t}, s\right)$. With this in mind, we define the one-period ahead constraint correspondence as the set-valued function mapping any $\left(W_{0}, p, q\right)$ to the corresponding one period-ahead constraint set, that is, the set of $\left(c_{0}, N_{0}, W_{1}\right) \in \mathbb{R}_{+} \times \mathcal{M}_{+} \times \mathbb{R}_{+}^{S}$ such that:

$$
\begin{aligned}
& q_{0} c_{0}+\sum_{s} q_{1}(s) W_{1}(s)+\int p_{0}(\delta) d N_{0}(\delta) \\
= & q_{0} W_{0}+\sum_{s} q_{1}(s) e_{1}(s)+\sum_{s} \int\left[q_{1}(s) \delta_{1}(s)+p_{1}(\delta \mid s)\right] d N_{0}(\delta),
\end{aligned}
$$

and, for all $s \in S$ :

$$
q_{1}(s) W_{1}(s) \geq q_{1}(s) e_{1}(s)+\int \theta_{i}(\delta)\left[q_{1}(s) \delta_{1}(s)+p_{1}(\delta \mid s)\right] d N_{0}(\delta)
$$

Equipped with this definition, we obtain:

\footnotetext{
${ }^{22}$ For the joint continuity, recall from Corollary 15.7 in Aliprantis and Border (2006) that the "evaluation function" $(p, N) \mapsto$ $\int p(\delta) d N(\delta)$ is jointly continuous in $(p, N)$.
} 
Lemma II.1 The one-period ahead constraint correspondence is lower hemi-continuous in $\left(W_{0}, p, q\right) \in \mathbb{R}_{+} \times \mathbb{N} A$.

Consider some sequence $\left(W_{0}^{\ell}, p^{\ell}, q^{\ell}\right) \rightarrow\left(W_{0}, p, q\right)$ and some $\left(c_{0}, N_{0}, W_{1}\right)$ in the one-period ahead constraint set given $\left(W_{0}, p, q\right)$. To prove lower hemi-continuity, we must find a sequence $\left(c_{0}^{\ell}, N_{0}^{\ell}, W_{1}^{\ell}\right) \rightarrow\left(c_{0}, N_{0}, W_{1}\right)$, such that for all $\ell$ large enough, $\left(c_{0}^{\ell}, N_{0}^{\ell}, W_{1}^{\ell}\right)$ belongs to one-period ahead constraint set given $\left(W_{0}^{\ell}, p^{\ell}, q^{\ell}\right)$. We distinguish four mutually exclusive cases.

Case 1: $q_{0} c_{0}>0$.

Then we pick the sequence $\left(c_{0}^{\ell}, N_{0}^{\ell}, W_{1}^{\ell}\right)$ such that:

$$
\begin{aligned}
& N_{0}^{\ell}=N_{0} \\
& q_{1}^{\ell}(s) W_{1}^{\ell}(s)=\max \left\{q_{1}^{\ell}(s) e_{1}(s)+\int \theta_{i}(\delta)\left[q_{1}^{\ell}(s) \delta_{1}(s)+p_{1}(\delta \mid s)\right] d N_{0}(\delta), q_{1}^{\ell}(s) W_{1}(s)\right\} \\
& q_{0}^{\ell} c_{0}^{\ell}+\int p_{0}(\delta) d N_{0}(\delta)+\sum_{s} q_{1}^{\ell}(s) W_{1}^{\ell}(s) \\
& \quad=q_{0}^{\ell} W_{0}^{\ell}+\sum_{s} q_{1}^{\ell}(s) e_{1}^{\ell}(s)+\sum_{s} \int\left(q_{1}^{\ell}(s) \delta_{1}(s)+p_{1}^{\ell}(\delta \mid s)\right) d N_{0}(\delta) .
\end{aligned}
$$

Since $q_{1}^{\ell}(s) W_{1}^{\ell}(s) \rightarrow q_{1}(s) W_{1}(s)$, it follows that $q_{0}^{\ell} c_{0}^{\ell} \rightarrow q_{0} c_{0}$, and so is positive for all $\ell$ large enough. Since all $q$ are strictly positive, it follows that $\left(c^{\ell}, N_{0}^{\ell}, W_{1}^{\ell}\right) \rightarrow\left(c, N_{0}, W_{1}\right)$.

Case 2: $q_{0} c_{0}=0$ and $q_{1}(s) W_{1}(s)=q_{1}(s) e_{1}(s)$ for each $s$.

Then it follows from the incentive constraints that

$$
\int \theta_{i}(\delta)\left[q_{1}(s) \delta_{1}(s)+p_{1}(\delta \mid s)\right] d N_{0}(\delta)=0
$$

Therefore, $N_{0}\left(\Delta_{0}^{+}\right)=0$, where $\Delta_{0}^{+}$is the set of trees with non-zero continuation dividend streams. Plugging this into the budget constraint shows that $W_{0}=0$. We then pick the sequence $c_{0}^{\ell}=W_{0}^{\ell}, N_{0}^{\ell}=N_{0}$ and $W_{1}^{\ell}(s)=e_{1}(s)$.

Case 3: $q_{0} c_{0}=0$ and $q_{1}(\hat{s}) W_{1}(\hat{s})>q_{1}(\hat{s}) e_{1}(\hat{s})+\int \theta_{i}(\delta)\left[q_{1}(\hat{s}) \delta_{1}(\hat{s})+p_{1}(\delta \mid \hat{s})\right] d N_{0}(\delta)$ for some $\hat{s}$.

Then we pick the sequence $c_{0}^{\ell}=c_{0}=0$ and $N_{0}^{\ell}=N_{0}$. For $s \neq \hat{s}$, we choose $W_{1}^{\ell}(s)$ according to (57). Then, we pick $W_{1}^{\ell}(\hat{s})$ so that the budget constraint (55) holds. By construction, $\left(c^{\ell}, N_{0}^{\ell}, W_{1}^{\ell}\right) \rightarrow\left(c, N_{0}, W_{1}\right)$. Moreover, for all $\ell$, the budget constraint hold, as well as the incentive constraints for $s \neq \hat{s}$. But by our maintained assumption the incentive it holds strictly in the limit for $s=\hat{s}$, so it holds for $\ell$ large enough too.

Case 4: $q_{0} c_{0}=0$, the incentive constraints bind for all $s$, and $q_{1}(\hat{s}) W_{1}(\hat{s})>q_{1}(\hat{s}) e_{1}(\hat{s})$ for some $\hat{s}$.

In this case, because $q_{1}(\hat{s}) W_{1}(\hat{s})>q_{1}(\hat{s}) e_{1}(\hat{s})$ but the incentive constraint binds for $\hat{s}$, it follows from the incentive constraint at $\hat{s}$ that

$$
\int \theta_{i}(\delta)\left[q_{1}(\hat{s}) \delta_{1}(\hat{s})+p_{1}(\delta \mid \hat{s})\right] d N_{0}(\delta)>0
$$

so that $N_{0}\left(\Delta^{+}\right)>0$. Now for some $\varepsilon^{\ell} \rightarrow 0$ to be determined later, pick $N_{0}^{\ell}=\left(1-\varepsilon^{\ell}\right) N_{0}$. Pick $W_{1}^{\ell}(s)$ so that the 
incentive constraint binds:

$$
\begin{aligned}
q_{1}^{\ell}(s) W_{1}^{\ell}(s) & =-\varepsilon^{\ell} \int \theta_{i}(\delta)\left[q_{1}^{\ell}(s) \delta_{1}(s)+p_{1}^{\ell}(\delta \mid s)\right] d N_{0}(\delta)+q_{1}^{\ell}(s) \hat{W}_{1}^{\ell}(s) \\
\text { where } q_{1}^{\ell}(s) \hat{W}_{1}^{\ell}(s) & \equiv q_{1}^{\ell}(s) e_{1}(s)+\int \theta_{i}(\delta)\left[q_{1}^{\ell}(s) \delta_{1}(s)+p_{1}^{\ell}(\delta \mid s)\right] d N_{0}(\delta)
\end{aligned}
$$

We now pick $\varepsilon^{\ell}$ and $c_{0}^{\ell}$ so that the bugdet constraint binds. After some algebra we obtain that, for the budget constraint to bind, $\varepsilon^{\ell}$ and $c_{0}^{\ell}$ must be chosen so that:

$$
\begin{aligned}
& q_{0}^{\ell} c_{0}^{\ell}-\varepsilon^{\ell} \int\left(p_{0}^{\ell}(\delta)-\sum_{s}\left(1-\theta_{i}(\delta)\right)\left[q_{1}^{\ell}(s) \delta_{1}(s)+p_{1}^{\ell}(\delta \mid s)\right]\right) d N_{0}(\delta) \\
= & W_{0}^{\ell}+\sum_{s} q_{1}^{\ell}(s) e_{1}(s)+\int\left(\sum_{s}\left[q_{1}^{\ell}(s) \delta_{1}(s)+p_{1}^{\ell}(\delta \mid s)\right]-p_{0}(\delta)\right) d N_{0}(\delta)-\sum_{s} q_{1}^{\ell}(s) \hat{W}_{1}^{\ell}(s)
\end{aligned}
$$

Since $\hat{W}_{1}^{\ell}(s) \rightarrow W_{1}(s), q_{0} c_{0}=0$, and since the budget constraint holds in the limit, it follows that the right-hand side goes to zero as $\ell \rightarrow \infty$. If we denote this right-hand side by $\eta^{\ell}$, then we can set $c_{0}^{\ell}$ and $\varepsilon^{\ell}$ to

$$
\begin{aligned}
& q_{0} c_{0}^{\ell}=\max \left\{\eta^{\ell}, 0\right\} \\
& \varepsilon^{\ell} \int\left[p_{0}^{\ell}(\delta)-\left(1-\theta_{i}(\delta)\right) \sum_{s}\left(q_{1}^{\ell}(s) \delta_{1}(s)+p_{1}^{\ell}(\delta \mid s)\right)\right] d N_{0}(\delta)=\min \left\{-\eta^{\ell}, 0\right\}
\end{aligned}
$$

Notice that the integral multiplying $\varepsilon^{\ell}$ is bounded away from zero for $\ell$ large enough. Indeed,it converges to

$$
\int\left[p_{0}(\delta)-\left(1-\theta_{i}(\delta)\right) \sum_{s}\left(q_{1}(s) \delta_{1}(s)+p_{1}(\delta \mid s)\right)\right] d N_{0}(\delta)
$$

which is strictly positive for two reasons: first, $(p, q) \in \mathbb{N A}$ so that the integrand is strictly positive over $\Delta^{+}$, and second $N_{0}\left(\Delta^{+}\right)>0$ so that the integral is strictly positive as well. This ensures that $\varepsilon^{\ell}$ is well defined for $\ell$ large enough, and goes to zero as $\ell$ goes to infinity.

\section{II.2 The multi-period constraint correspondence}

The proof now follows by recursive application of Lemma II.1 in the previous subsection. Namely, consider some $\left(W_{0}, p, q\right) \in \mathbb{N A}$, some $(c, N) \in \Gamma_{i}\left(W_{0}, p, q\right)$. Let $W$ denote the sequence of cash on hand associated with this plan. Consider any sequence $\left(W_{0}^{\ell}, p^{\ell}, q^{\ell}\right) \rightarrow\left(W_{0}, p, q\right)$. Lemma II.1 allows to construct sequences $c_{0}^{\ell}\left(s^{0}\right), W_{1}^{\ell}\left(s^{0}, s_{1}\right), N_{0}^{\ell}\left(s^{0}\right)$ converging to $W_{0}, c_{0}\left(s^{0}\right), N_{0}\left(s^{0}\right)$ and $W_{1}\left(s^{0}, s_{1}\right)$. satisfying the one-period ahead budget constraint. Now repeat this step at each node $\left(t, s^{t}\right)$ until reaching the second to last period, $T-1$.

\section{Proof of Proposition A.2}

\section{III.1 Unbounded constraint set}

We first establish that: 
Lemma III.1 Take any $(p, q) \in \overline{\mathbb{N A}} \backslash \mathbb{N A}$. Then for any sequence $\left(p^{\ell}, q^{\ell}\right)$ in NA converging to $(p, q)$, there exists some agent $i$ and some sequence $\left(c_{i}^{\ell}, N_{i}^{\ell}\right) \in \Gamma_{i}\left(W_{0}^{\ell}, p^{\ell}, q^{\ell}\right)$, where $W_{0}^{\ell}$ is defined by (9), such that $\left\|c_{i}^{\ell}\right\| \rightarrow \infty$.

Lemma A.1 implies that, if $(p, q) \in \overline{\mathbb{N} A} \backslash \mathbb{N} A$, either

$$
q_{t}\left(s^{t}\right)=0
$$

for some $\left(t, s^{t}\right)$, or there exists some agent $i$, some $\left(t, s^{t}\right)$ and some $\delta \in \Delta_{t}^{+}\left(s^{t}\right)$, such that

$$
p_{t}\left(\delta \mid s^{t}\right)=\left(1-\theta_{i}(\delta)\right) \sum_{s}\left[q_{t+1}\left(s^{t}, s\right) \delta_{t+1}\left(s^{t}, s\right)+p_{t+1}\left(\delta \mid s^{t}, s\right)\right]
$$

For what follows let us denote by

$$
T_{i}^{\ell} \equiv \alpha_{i} \int\left[q_{0}^{\ell}\left(s^{0}\right) \delta_{0}\left(s^{0}\right)+p_{0}^{\ell}\left(\delta \mid s^{0}\right)\right] d \bar{N}(\delta)
$$

the initial tree wealth of the agent. Notice that $T_{i}^{\ell}$ has a strictly positive limit as $\ell \rightarrow \infty$. Ineeed, for any price system $(p, q) \in \overline{\mathbb{N A}}$, iterating forward on the no-arbitrage condition (13) (with a weak inequality) starting at time $t=0$, implies that

$$
q_{0}\left(s^{0}\right) \delta_{0}\left(s^{0}\right)+p_{0}\left(\delta \mid s^{0}\right) \geq \sum_{\left(t, s^{t}\right)}\left(1-\theta_{i}(\delta)\right)^{t} q_{t}\left(s^{t}\right) \delta_{t}\left(s^{t}\right)
$$

Integrating against the aggregate supply we obtain that:

$$
\int\left[q_{0}\left(s^{0}\right) \delta_{0}\left(s^{0}\right)+p_{0}\left(\delta \mid s^{0}\right)\right] d \bar{N}(\delta) \geq \sum_{\left(t, s^{t}\right)}\left(1-\theta_{i}(\delta)\right)^{t} \int q_{t}\left(s^{t}\right) \delta_{t}\left(s^{t}\right) d \bar{N}(\delta)>0
$$

given our assumption that the aggregate dividend is strictly positive in all state and since our normalization (33) ensures at least one of the consumption prices, $q_{t}\left(s^{t}\right)$, must be strictly positive. It thus follows that the value of every agent's initial tree wealth is strictly positive in the limit $(p, q)$, and bounded away from zero near the limit.

Case 1. Suppose that the time-zero price of consumption is zero at some node, $\left(t, s^{t}\right), q_{t}\left(s^{t}\right)=0$. Since all elements of the sequence $\left(p^{\ell}, q^{\ell}\right)$ belong to $\mathbb{N A}, q_{u}^{\ell}\left(s^{u}\right)>0$ for each node $\left(u, s^{u}\right)$. Hence, an agent can save her initial tree wealth from node to node until $\left(t, s^{t}\right)$, at which point she can consume the amount $c_{i t}^{\ell}\left(s^{t}\right)$ solving $q_{t}^{\ell}\left(s^{t}\right) c_{i t}^{\ell}\left(s^{t}\right)=q_{t}^{\ell}\left(s^{t}\right) e_{i t}\left(s^{t}\right)+T_{i}^{\ell}$. At all other node, the agent can consume hand-to-mouth, $c_{i u}^{\ell}\left(s^{u}\right)=e_{i u}\left(s^{u}\right) .{ }^{23}$ Since $q_{t}^{\ell}\left(s^{t}\right) \rightarrow 0$, and since $T_{i}^{\ell}$ remains bounded away from zero for all $\ell$ large enough, it follows that $c_{i t}^{\ell}\left(s^{t}\right) \rightarrow \infty$.

Case 2. Now suppose that the price of consumption is strictly positive at all nodes but (59) holds for some $i$, some $\left(t, s^{t}\right)$ and some $\delta \in \Delta_{t}^{+}\left(s^{t}\right)$. Then proceeding exactly as above the agent can choose cash on hand equal to $q_{t}^{\ell}\left(s^{t}\right) e_{i t}\left(s^{t}\right)+T_{i}^{\ell}$ for node $\left(t, s^{t}\right)$. At this point she can purchase an amount $n^{\ell}>0$ of tree $\delta$ (formally, this portfolio is a discrete measure concentrated at $\delta$ ) and partially finance the purchase by selling the tree pledgeable payoff. If

\footnotetext{
${ }^{23}$ The corresponding plan $\left(c_{i}, N_{i}\right)$ is the following. Set $N_{i u}^{\ell}\left(s^{u}\right)=0$ for all node. For all nodes $\left(u, s^{u}\right) \preceq\left(t, s^{t}\right)$, set $q_{u}^{\ell}\left(s^{u}\right) W_{i u}^{\ell}\left(s^{u}\right)=q_{u}^{\ell}\left(s^{u}\right) e_{i u}\left(s^{u}\right)+T_{i}^{\ell}$. For all other node, set $W_{i u}^{\ell}\left(s^{u}\right)=e_{i u}\left(s^{u}\right)$. For all nodes $\left(u, s^{u}\right) \neq\left(t, s^{t}\right)$, set $c_{i u}^{\ell}\left(s^{u}\right)=$ $e_{i u}\left(s^{u}\right)$. For $\left(t, s^{t}\right)$, pick $c_{i t}^{\ell}\left(s^{t}\right)$ solving $q_{t}^{\ell}\left(s^{t}\right) c_{i t}^{\ell}\left(s^{t}\right)=q_{t}^{\ell}\left(s^{t}\right) e_{i t}\left(s^{t}\right)+T_{i}^{\ell}$.
} 
the agent consumes hand to mouth at this node, $c_{t}^{\ell}\left(s^{t}\right)=e_{i t}\left(s^{t}\right)$, then:

$$
\begin{aligned}
& n^{\ell}\left(p_{t}^{\ell}\left(\delta \mid s^{t}\right)-\left(1-\theta_{i}(\delta)\right) \sum_{s}\left(q_{t+1}^{\ell}\left(s^{t}, s\right) \delta_{t+1}\left(s^{t}, s\right)+p_{t+1}^{\ell}\left(\delta \mid s^{t}, s\right)\right)\right)=T_{i}^{\ell} \\
& q_{t+1}^{\ell}\left(s^{t}, s\right) W_{i t+1}^{\ell}\left(s^{t}, s\right)=q_{t+1}^{\ell}\left(s^{t}, s\right) e_{t+1}\left(s^{t}, s\right)+n^{\ell} \theta_{i}(\delta)\left(q_{t+1}^{\ell}\left(s^{t}, s\right) \delta_{t+1}\left(s^{t}, s\right)+p_{t+1}^{\ell}\left(\delta \mid s^{t}, s\right)\right) \\
& c_{i t+1}^{\ell}\left(s^{t}, s\right)=W_{i t+1}^{\ell}\left(s^{t}, s\right) .
\end{aligned}
$$

Clearly, $n^{\ell} \rightarrow \infty$. Since $\delta \in \Delta_{t}^{+}\left(s^{t}\right)$, it follows from the no-arbitrage relationship (36) holding with weak equality together with our maintained assumption that $q_{t}\left(s^{t}\right)>0$ at all nodes, that the tree payoff is strictly positive in some state $\hat{s}$. It follows that $c_{t+1}^{\ell}\left(s^{t}, \hat{s}\right) \rightarrow \infty$.

\section{III.2 Unbounded optimal demands}

We now show that an unbounded constraint set implies an unbounded demand. Precisely, take any $\left(c_{i}^{\ell}, N_{i}^{\ell}\right) \in$ $Z_{i}\left(p^{\ell}, q^{\ell}\right)$ and assume it is bounded in consumption. Use the previous lemma to generate another sequence $\left(\hat{c}_{i}^{\ell}, \hat{N}_{i}^{\ell}\right) \in$ $\Gamma_{i}\left(W_{i 0}^{\ell}, p^{\ell}, q^{\ell}\right)$, where $W_{i 0}^{\ell}$ is defined according to $(9)$, that is unbounded in consumption. Since $\left\|\hat{c}_{i}^{\ell}\right\| \geq 1$ for all $\ell$ large enough, it follows by convexity that, for all $\ell$ large enough, the plan

$$
\left(1-\frac{1}{\left\|\hat{c}_{i}^{\ell}\right\|}\right)\left(c_{i}^{\ell}, N_{i}^{\ell}\right)+\frac{1}{\left\|\hat{c}_{i}^{\ell}\right\|}\left(\hat{c}_{i}^{\ell}, \hat{N}_{i}^{\ell}\right)
$$

belongs to the constraint set $\Gamma_{i}\left(W_{i 0}^{\ell}, p^{\ell}, q^{\ell}\right)$, where $W_{i 0}^{\ell}$ is defined according to (9). Moreover, since the norm of $\hat{c}_{i}^{\ell} /\left\|\hat{c}_{i}^{\ell}\right\|$ is equal to one, at least one of its coordinate must remain bounded away from zero. It then follows by continuity that the above convex combination must yield strictly higher intertemporal utility than $\left(c_{i}^{\ell}, N_{i}^{\ell}\right)$ for all $\ell$ large enough, and we have reached a contradiction.

\section{Proof of Proposition A.3}

Necessity. Suppose $\left(c_{i}, N_{i}\right)$ solves the agent's problem together with some cash-on-hand plan $W_{i}$. Since utility is strictly increasing, $\left(c_{i}, N_{i}\right)$, it also solves the "relaxed" problem in which all sequential budget constraints are only required to hold with a weak inequality. Since the constraint set is defined by a finite number of inequality constraints, the associated positive cone has interior points. Since the agent starts with strictly positive tree wealth, it can guarantee strictly positive cash-on-hand at all nodes, so there is some plan for consumption, tree portfolio, and cash-on-hand such that all constraints hold with strict inequalities. It then follows from Theorem 1 , chapter 8.3 in Luenberger (1969) that there exists positive multipliers $\lambda$ and $\mu$, as shown in the Proposition, such that the associated Lagrangian is maximized at $\left(c_{i}, N_{i}, W_{i}\right)$, and such that the associated complementarity slackness conditions hold. The result follows.

Sufficiency. This follows by a standard optimality-verification argument.

From Proposition A.3 to the first-order conditions shown in the text. The first-order condition (16) and (17) shown in the text obtain by re-defining $\lambda_{i t}\left(s^{t}\right) \equiv \hat{\lambda}_{i t}\left(s^{t}\right) q_{t}\left(s^{t}\right)$ and $\mu_{i t}\left(s^{t}\right) \equiv \hat{\mu}_{i t}\left(s^{t}\right) q_{t}\left(s^{t}\right)$. The first-order 
condition (19) requires some algrebraic manipulations. First, (40) implies that

$$
\sum_{s}\left(1-\frac{\theta_{i}(\delta) \hat{\mu}_{i t+1}\left(s^{t}, s\right)}{\hat{\lambda}_{i t}\left(s^{t}\right)}\right)\left[q_{t+1}\left(s^{t}, s\right) \delta_{t+1}\left(s^{t}, s\right)+p_{t+1}\left(\delta \mid s^{t}, s\right)\right] \leq p_{t}\left(\delta \mid s^{t}\right)
$$

with an equality if $d N_{i t}\left(\delta \mid s^{t}\right)>0$. Now dividing through both sides by $q_{t}\left(s^{t}\right)$ and keeping in mind that $Q_{t+1}\left(s^{t}, s\right)=$ $q_{t+1}\left(s^{t}, s\right) / q_{t}\left(s^{t}\right)$, this inequality writes:

$$
\sum_{s}\left(1-\frac{\theta_{i}(\delta) \hat{\mu}_{i t+1}\left(s^{t}, s\right)}{\hat{\lambda}_{i t}\left(s^{t}\right)}\right) Q_{t+1}\left(s^{t}, s\right)\left[\delta_{t+1}\left(s^{t}, s\right)+P_{t+1}\left(\delta \mid s^{t}, s\right)\right] \leq P_{t}\left(\delta \mid s^{t}\right),
$$

The term in parenthesis can be then rewritten:

$$
\begin{aligned}
1-\frac{\theta_{i}(\delta) \hat{\mu}_{i t+1}\left(s^{t}, s\right)}{\hat{\lambda}_{i t}\left(s^{t}\right)} & =1-\theta_{i}(\delta)+\theta_{i}(\delta) \frac{\hat{\lambda}_{i t}\left(s^{t}\right)-\hat{\mu}_{i t+1}\left(s^{t}, s\right)}{\hat{\lambda}_{i t}\left(s^{t}\right)} \\
& =1-\theta_{i}(\delta)+\theta_{i}(\delta) \frac{\hat{\lambda}_{i t+1}\left(s^{t+1}\right)}{\hat{\lambda}_{i t}\left(s^{t}\right)} \\
& =1-\theta_{i}(\delta)+\theta_{i}(\delta) \frac{\lambda_{i t+1}\left(s^{t+1}\right)}{Q_{t+1}\left(s^{t}, s\right) \lambda_{i t}\left(s^{t}\right)}
\end{aligned}
$$

where the equality on the second line follows from (38), and the equality on the third line follows from the definition of $\lambda_{i t}\left(s^{t}\right)$ and $\mu_{i t}\left(s^{t}\right)$. The first-order condition (19) follows.

\section{Proof of Proposition A.4}

Using our basis notation, the sequential budget constraint of agent $i$ writes, for all $t<T$

$$
\begin{aligned}
& q_{t}\left(s^{t}\right) c_{i t}\left(s^{t}\right)+\sum_{s^{\prime}} q_{t+1}\left(s^{t}, s\right) W_{i t+1}\left(s^{t}, s\right) \\
= & q_{t}\left(s^{t}\right) W_{i t}\left(s^{t}\right)+\sum_{s} q_{t+1}\left(s^{t}, s\right) e_{i t+1}\left(s^{t}, s\right)+\int b_{t}\left(\delta \mid s^{t}\right) d N_{i t}\left(\delta \mid s^{t}\right) .
\end{aligned}
$$

Adding up all the constraint until $T-1$, as well as the time- $T$ constraint $W_{i T}\left(s^{T}\right)=c_{i T}\left(s^{T}\right)$, all the $W_{i t}\left(s^{t}\right)$ cancel out except for the first one, $W_{i 0}\left(s^{0}\right)=e_{i 0}\left(s^{0}\right)+\alpha_{i} \int\left(q_{0}\left(s^{0}\right) \delta_{0}\left(s^{0}\right)+p_{0}\left(\delta \mid s^{0}\right)\right) d \bar{N}(\delta)$. We thus obtain the intertemporal budget constraint:

$$
\sum_{\left(t, s^{t}\right)} q_{t}\left(s^{t}\right) c_{i t}\left(s^{t}\right)=\alpha_{i} \int\left(q_{0}\left(s^{0}\right) \delta_{0}\left(s^{0}\right)+p_{0}\left(\delta \mid s^{0}\right)\right) d \bar{N}(\delta)+\sum_{\left(t, s^{t}\right)} q_{t}\left(s^{t}\right) e_{i t}\left(s^{t}\right)+\sum_{\left(t, s^{t}\right), t<T} \int b_{t}\left(\delta \mid s^{t}\right) d N_{i t}\left(\delta \mid s^{t}\right)
$$

Now recall that, by definition of the basis:

$$
p_{t}\left(\delta \mid s^{t}\right)=\sum_{s^{\prime}}\left(q_{t+1}\left(s^{t}, s^{\prime}\right) \delta_{t+1}\left(s^{t}, s^{\prime}\right)+p_{t+1}\left(\delta \mid s^{t}, s^{\prime}\right)\right)-b_{t}\left(\delta \mid s^{t}\right)
$$


for all $\left(t, s^{t}\right), t<T$. Iterating forward on this equation starting at the initial node $\left(0, s^{0}\right)$, we obtain that:

$$
q_{0}\left(s^{0}\right) \delta_{0}\left(s^{0}\right)+p_{0}\left(\delta \mid s^{0}\right)=\sum_{\left(t, s^{t}\right)} q_{t}\left(s^{t}\right) \delta_{t}\left(s^{t}\right)-\sum_{\left(t, s^{t}\right), t<T} b_{t}\left(\delta \mid s^{t}\right)
$$

that is, tree prices are equal to the present value of all of their future dividends, net of the sum of all their bases. Plugging back into the intertemporal budget constraint we obtain:

$$
\sum_{\left(t, s^{t}\right)} q_{t}\left(s^{t}\right) c_{i t}\left(s^{t}\right)=\sum_{\left(t, s^{t}\right)} q_{t}\left(s^{t}\right)\left(e_{i t}\left(s^{t}\right)+\alpha_{i} \int \delta_{t}\left(s^{t}\right) d \bar{N}(\delta)\right)+\sum_{\left(t, s^{t}\right), t<T} \int b_{t}\left(\delta \mid s^{t}\right)\left(d N_{i t}\left(\delta \mid s^{t}\right)-\alpha_{i} d \bar{N}(\delta)\right)
$$

and the result follows by adding up these intertemporal constraints across all $i$ and using that $\sum_{i} \alpha_{i}=1$.

\section{V.1 Lower hemi-continuity for the multi-period constraint set bounded by $B$}

To show lower hemi-continuity at $\left(W_{0}, p, q\right)$ consider any $(c, n) \in \Gamma_{i}\left(W_{0}, p, q\right) \cap B$ and any $\left(W_{0}^{\ell}, p^{\ell}, q^{\ell}\right) \rightarrow\left(W_{0}, p, q\right)$. Since the correspondence $\Gamma_{i}\left(W_{0}, p, q\right)$ is lower hemi-continuous, we already know that there is a sequence $\left(c^{\ell}, n^{\ell}\right) \in$ $\Gamma_{i}\left(W_{0}^{\ell}, p^{\ell}, q^{\ell}\right)$ converging to $(c, n)$. But this sequence may lie outside $B$. To bring each element of the sequence inside $B$, take a convex combination of $\left(c^{\ell}, n^{\ell}\right)$ with the hand-to-mouth plan, which we denote $\left(\hat{c}^{\ell}, \hat{n}^{\ell}\right): \hat{n}_{t}\left(s^{t}\right)=0$, $\hat{c}_{0}^{\ell}\left(s^{0}\right)=W_{0}^{\ell}\left(s^{0}\right), \hat{c}_{t}\left(s^{t}\right)=\hat{W}_{t}^{\ell}\left(s^{t}\right)=e_{t}\left(s^{t}\right)$ for $t>0$. Since $\left(\hat{c}^{\ell}, \hat{n}^{\ell}\right) \in B$, there exists $\lambda \in[0,1]$ such that:

$$
\lambda\left(\hat{c}^{\ell}, \hat{n}^{\ell}\right)+(1-\lambda)\left(c^{\ell}, n^{\ell}\right) \in B
$$

Let $\lambda^{\ell}$ be the smallest $\lambda$ such that this is the case. Recall that, for $(\hat{c}, \hat{n})$, the right-hand-side inequalities defining $B$, (43) and (44), hold strictly. Together with the fact that $\left(\hat{c}^{\ell}, \hat{n}^{\ell}\right) \rightarrow(\hat{c}, \hat{n})$ and $\left(c^{\ell}, n^{\ell}\right) \rightarrow(c, n) \in B$, we obtain that, for any $\lambda>0, \lambda\left(\hat{c}^{\ell}, \hat{n}^{\ell}\right)+(1-\lambda)\left(c^{\ell}, n^{\ell}\right)$ satisfies these right-hand-side inequality strictly for all $\ell$ large enough. Therefore, $\lambda^{\ell} \leq \lambda$ for all $\ell$ large enough. Since this is true for all $\lambda>0$, we obtain that $\lambda^{\ell} \rightarrow 0$ and have found the desired sequence.

\section{V.2 Proof of Proposition B.1}

The fixed point problem has a solution. By an application of the Theorem of the Maximum (see Theorem 3.6 in Stokey and Lucas, 1989), this correspondence is compact valued and upper hemi-continuous. ${ }^{24}$ It is also convex valued because the constraints sets are convex and the objective concave. Hence, an application of the Kakutani fixed point theorem (see Theorem M.I.2. p.953 in Mas-Colell, Whinston and Green, 1995) shows that it has a fixed point, $\left(p^{\varepsilon}, q^{\varepsilon},\left(c_{i}^{\varepsilon}, n_{i}^{\varepsilon}\right)_{i \in I}\right)$. By Walras Law stated in Proposition A.4, the maximized price player objective must be equal to zero.

The $B$ constraints are not binding. Notice that, given any $q$, it is always feasible for the price player to choose tree prices with zero bases, $\mathfrak{b}\left(p, q^{\varepsilon}\right)=0$. Therefore, the price player's value is weakly negative when she sets

\footnotetext{
${ }^{24}$ When $u_{i}(c)$ is unbounded below, the Theorem does not apply directly because the domain of the utility function is $(0, \infty)$ instead of $[0, \infty)$, so the constraint set is not compact. But one can restrict the domain to consumptions that are bounded away from zero by some constant. Indeed, since aggregate dividends are strictly positive at all nodes and Arrow security prices add up to one, agents' initial wealth can be bounded away from zero (see equation (60)). Therefore, purchasing Arrow securities only, whose prices are bounded above by 1 , the agent can guarantee herself positive consumption at all nodes: formally, there exists some $\left(c_{i}, N_{i}\right)$, such that $c_{i t}\left(s^{t}\right)>0$ at all $\left(t, s^{t}\right)$ and $N_{i}=0$, which is budget feasible and incentive compatible for all prices $\left(p^{\varepsilon}, q^{\varepsilon}\right)$. This places a lower bound on the agent's maximum attainable utility and, when utility is unbounded below, a strictly positive lower bound on the agent's consumption. Hence we can restrict the domain, as claimed.
} 
$q_{t}\left(s^{t}\right)=\bar{q}$, for some constant $\bar{q}$ such that consumption prices add up to one, and chooses $p$ such that $\mathbb{b}(p, q)=0$. This implies that:

$$
\sum_{\left(t, s^{t}\right)} \sum_{i} c_{i t}\left(s^{t}\right) \leq \sum_{\left(t, s^{t}\right)} \omega_{t}\left(s^{t}\right)
$$

so that, at the fixed point, the constraint (43) is slack for consumption.

Then, we argue that the constraint (44) is slack for tree holdings as well. This is evident if if $\sum_{i} n_{i k t}\left(s^{t}\right) \leq \bar{N}_{k}$. If $\sum_{i} n_{i k t}\left(s^{t}\right)>\bar{N}_{k}$ for some tree $k$ and node $\left(t, s^{t}\right)$, then $\mathfrak{b}_{k t}\left(s^{t} \mid p^{\varepsilon}, q^{\varepsilon}\right)=0$, otherwise, the price player could increase its value by reducing the basis of tree $k$ at node $\left(t, s^{t}\right)$ to zero, and keep all other bases the same. To see that such reduction in basis is consistent with the no-arbitrage conditions (i.e., the corresponding price system remains in the constraint set of the price player) let us consider any price system $(p, q) \in \mathbb{N A}^{\varepsilon}$ and show that the price system remains in $N A^{\varepsilon}$ if one reduces the basis of any specific tree to zero at any node $\left(t, s^{t}\right)$. Indeed, consider the tree prices $\hat{p}$ obtained by inverting the basis formula

$$
\hat{p}_{t}\left(s^{t}\right)=\sum_{s^{\prime}}\left(q_{t+1}\left(s^{t}, s^{\prime}\right) \delta_{t+1}\left(s^{t}, s^{\prime}\right)+\hat{p}_{t+1}\left(s^{t}, s^{\prime}\right)\right)-\hat{b}_{t}\left(s^{t}\right)
$$

from $\hat{p}_{T}\left(s^{T}\right)=0$, setting $\hat{b}_{k t}\left(t, s^{t}\right)=0$, but otherwise keeping all other bases the same, $\hat{b}_{j u}\left(s^{u}\right)=b_{j u}\left(s^{u}\right)$ for $j \neq k$ or $\left(u, s^{u}\right) \neq\left(t, s^{t}\right)$. For all trees $j \neq k$, the tree prices remain the same. For tree $k$, the price is the same at all nodes $\left(u, s^{u}\right) \succ\left(t, s^{t}\right)$, and it increases strictly by $b_{k t}\left(s^{t}\right)$ at all nodes $\left(u, s^{u}\right) \preceq\left(t, s^{t}\right)$. But this relaxes the constraint

$$
\begin{aligned}
\hat{p}_{k u}\left(s^{u}\right) \geq\left(1-\theta_{i k}+\varepsilon\right) \sum_{s^{\prime}}\left(q_{u+1}\left(s^{u}, s^{\prime}\right) \delta_{k u+1}\left(s^{u}, s^{\prime}\right)+\hat{p}_{k u+1}\left(s^{u}, s^{\prime}\right)\right) \\
\Leftrightarrow \quad \hat{b}_{k u}\left(s^{u}\right) \leq\left(\theta_{i k}-\varepsilon\right) \sum_{s^{\prime}}\left(q_{u+1}\left(s^{u}, s^{\prime}\right) \delta_{k u+1}\left(s^{t}, s^{\prime}\right)+\hat{p}_{k u+1}\left(s^{t}, s^{\prime}\right)\right)
\end{aligned}
$$

since $\hat{b}_{k u}\left(u, s^{u}\right) \leq b_{k u}\left(s^{u}\right)$ and $\hat{p}_{k u}\left(s^{u}\right) \geq p_{k u}\left(s^{u}\right)$.

Now, since $\mathfrak{b}_{k t}\left(s^{t} \mid p, q\right)=0$, then the agent can reduce its holdings of tree $k$ arbitrarily, keep the same budget, and relax her incentives constraint. Hence, (44) is not binding either.

For $\varepsilon$ small enough, the constraint (45) and (46) are not binding. The finding that both (43) and (44) are slack implies, together with the the concavity of the objective and the convexity of the constraint set, that $\left(c_{i}^{\varepsilon}, n_{i}^{\varepsilon}\right)$ solves the agent's problem given $\left(p^{\varepsilon}, q^{\varepsilon}\right)$, but unconstrained by $B$, that is, subject to $\left(c_{i}, n_{i}\right) \in \Gamma_{i}\left(W_{0}^{\varepsilon}, p^{\varepsilon}, q^{\varepsilon}\right)$ only, where $W_{0}^{\varepsilon}$ is defined by (9). Moreover, all the $c_{i}^{\varepsilon}$ are uniformly bounded. Now consider any sequence $\varepsilon^{\ell} \rightarrow 0$, an associated sequence of fixed points $\left(p^{\ell}, q^{\ell}\right)$ and $\left(c_{i}^{\ell}, n_{i}^{\ell}\right)_{i \in I}$. Given that all of these sequences are bounded we can extract convergent subsequences (but keep the same notation). By Proposition A.2, the limit price cannot belong to $\overline{N A} \backslash \mathbb{N A}$ otherwise the associated consumption sequence of some agent would be unbounded. Hence, the limit of the price sequence belongs to NA. This implies that, for $\ell$ large enough, the constraint (45) is not binding, and the constraint (46) is not binding either for trees with non-zero continuation dividends.

Tree-market clearing. For a tree with zero continuation dividend at $\left(t, s^{t}\right)$, the no-arbitrage conditions imply that current and future prices are zero. This makes the tree is irrelevant in all current and future budget and incentive constraints, and implies that any demand is optimal. Therefore, we can pick the demand so as to clear the market. 
For a tree with non-zero continuation dividend, consider the corresponding term in the price player's objective

$$
-b_{k t}\left(s^{t}\right)\left(\sum_{i} n_{i k t}\left(s^{t}\right)-\bar{N}_{k}\right)
$$

Now, we have shown that, for $\varepsilon$ small enough, the constraint (46) of the price player is not binding for all $\left(u, s^{u}\right) \preceq$ $\left(t, s^{t}\right)$. This means that the price player can, without violating its no-arbitrage constraints, increase slightly the basis of the tree at time $\left(t, s^{t}\right)$ by reducing $p_{k t}\left(s^{t}\right)$, and keep all other bases the same by corresponding reductions in $p_{k u}\left(s^{u}\right)$ at $\left(u, s^{u}\right) \prec\left(t, s^{t}\right)$. Since this cannot increase the price player's objective, it follows that $\sum_{i} n_{i k t}\left(s^{t}\right) \geq \bar{N}_{k}$.

If, at node $\left(t, s^{t}\right)$, the basis of tree $k$ is strictly positive, then by the same argument used just above to show that the $B$ constraint is not binding, the price player can, without violating its no-arbitrage constraints, reduce the basis $b_{k t}\left(s^{t}\right)$ and keep all other bases the same. Since this cannot improve the price player's objective, we obtain that $\sum_{i} n_{i k t}\left(s^{t}\right) \leq \bar{N}_{k}$, and we are done.

If, at node $\left(t, s^{t}\right)$, the basis of tree $k$ is zero, then reducing the demand of the tree leaves the budget constraint the same, and relaxes the incentive constraint. Hence we can adjust the demand of each agent in such a way that $\sum_{i} n_{i k t}\left(s^{t}\right)=\bar{N}_{k}$.

Consumption-market clearing. For $\ell$ large enough, the constraint (45) is not binding, and the constraint (46) is not binding for trees with non-zero continuation dividend. Hence, it is feasible for the price player to increase and decrease slightly any two coordinates of $q_{t}^{\ell}\left(s^{t}\right)$ and keep all the bases the same. Since this cannot increase the price player's objective, we conclude that $\sum_{i} c_{i t}^{\ell}\left(s^{t}\right)-\omega_{t}\left(s^{t}\right)$ is constant across all nodes.

Now recall that, at the fixed point, Walras Law implies that the price player's objective must be zero. Since we know that the tree market clears, this writes:

$$
\sum_{\left(t, s^{t}\right)} q_{t}^{\ell}\left(s^{t}\right)\left[\sum_{i} c_{i t}^{\ell}\left(s^{t}\right)-\omega_{t}\left(s^{t}\right)\right]=0 .
$$

But since $\sum_{i} c_{i t}^{\ell}\left(s^{t}\right)-\omega_{t}\left(s^{t}\right)$ is constant across all nodes, it follows that it must be equal to zero.

\section{V.3 Proof of Lemma B.1}

Proceeding as in Proposition A.3, we obtain that the first-order conditions with respect to $n$, for the economy with finitely many trees write:

$$
v_{i k t}\left(s^{t}\right)-p_{k t}\left(s^{t}\right) \leq 0, \text { with } "=" \text { if } n_{i k t}\left(s^{t}\right)>0,
$$

where

$$
v_{i k t}\left(s^{t}\right) \equiv \sum_{s}\left(1-\frac{\theta_{i}\left(\delta_{k}\right) \mu_{i t+1}\left(s^{t}, s\right)}{\lambda_{i t}\left(s^{t}\right)}\right)\left[q_{t+1}\left(s^{t}, s\right) \delta_{k t+1}\left(s^{t}, s\right)+p_{k t+1}\left(s^{t}, s\right)\right] .
$$

Now given $q$ and the Lagrange multiplier's $\lambda$ and $\mu$, let us define the functions $\hat{p}_{t}\left(\delta \mid s^{t}\right)$ and $\hat{v}_{i t}\left(\delta \mid s^{t}\right)$ and as follows, backwards from time $T-1$ :

$$
\hat{p}_{t}\left(\delta \mid s^{t}\right)=\max _{i} \hat{v}_{i t}\left(\delta \mid s^{t}\right)
$$


where

$$
\hat{v}_{i t}\left(\delta \mid s^{t}\right)=\sum_{s}\left(1-\frac{\theta_{i}(\delta) \mu_{i t+1}\left(s^{t}, s\right)}{\lambda_{i t}\left(s^{t}\right)}\right)\left[q_{t+1}\left(s^{t}, s\right) \delta_{t+1}\left(s^{t}, s\right)+\hat{p}_{t+1}\left(\delta \mid s^{t}, s\right)\right]
$$

with the convention that $p_{T}=0$.

By construction, $\hat{p}_{t}\left(\delta_{k} \mid s^{t}\right)=p_{k t}\left(s^{t}\right)$. Moreover, it is clear that the first-order necessary and sufficient conditions continue to hold when agents can choose any tree in $\Delta$. Finally, the price functionals can be viewed as a jointly continous functions of $\delta \in \Delta$ and of the finitely many multipliers ratios $\mu_{i t+1}\left(s^{t}, s\right) / \lambda_{i t}\left(s^{t}\right) \in[0,1]$, for all $i$, all $t \geq 0$, all $s^{t} \in S^{t}$, and $s \in S$. This function is uniformly continuous since its domain is compact. This implies that, when viewed as function of $\delta$ only, the price functionals belong to an equi-continuous family.

\section{Proof of Corollary 1}

Suppose we allow short-selling of trees subject to the incentive constraint (15) as explained prior to Corollary 1. Write the sequential budget constraints as in equation (3) (i.e. do not make the cash-on-hand change of variable), using deflated time-zero prices $(p, q)$. Assume that the pledgeability parameter depends on assets and agents, $\theta_{i}(s)$ for Arrow securities and $\theta_{i}(\delta)$ for trees held by agent $i$. Proceeding as in Section A.3, the first-order necessary and sufficient conditions with respect to $c_{i t}\left(s^{t}\right), a_{i t+1}^{+}\left(s^{t}, s\right), a_{i t+1}^{-}\left(s^{t}, s\right), N_{i t}^{+}\left(s^{t}\right)$, and $N_{i t}^{-}\left(s^{t}\right)$, write:

$$
\begin{gathered}
\beta^{t} \pi_{t}\left(s^{t}\right) u_{i}^{\prime}\left(c_{t}\left(s^{t}\right)\right) \leq \hat{\lambda}_{i t}\left(s^{t}\right) q_{t}\left(s^{t}\right) \text { with" }=" \text { if } c_{i t}\left(s^{t}\right)>0 \\
\hat{\lambda}_{i t+1}\left(s^{t}, s\right)+\left(1-\theta_{i}\left(s_{t}\right)\right) \hat{\mu}_{i t+1}\left(s^{t}, s\right) \leq \hat{\lambda}_{i t}\left(s^{t}\right) \text { with " }=" \text { if } a_{i t+1}^{+}\left(s^{t}, s\right)>0 \\
\hat{\lambda}_{i t+1}\left(s^{t}, s\right)+\hat{\mu}_{i t+1}\left(s^{t}, s\right) \geq \hat{\lambda}_{i t}\left(s^{t}\right) \text { with " }=" \text { if } a_{i t+1}^{-}\left(s^{t}, s\right)>0 \\
\int\left[\hat{v}_{i t}^{+}\left(\delta \mid s^{t}\right)-p_{t}\left(\delta \mid s^{t}\right)\right] d M(\delta) \leq 0 \text { for all } M \in \mathcal{M}_{+} \text {with " }=" \text { if } M=N_{i t}^{+}\left(s^{t}\right) \\
\int\left[p_{t}\left(\delta \mid s^{t}\right)-\hat{v}_{i t}^{-}\left(\delta \mid s^{t}\right)\right] d M(\delta) \leq 0 \text { for all } M \in \mathcal{M}_{+} \text {with " }=" \text { if } M=N_{i t}^{-}\left(s^{t}\right)
\end{gathered}
$$

where $\hat{v}_{i t}^{+}\left(\delta \mid s^{t}\right)$ and $\hat{v}_{i t}^{-}\left(\delta \mid s^{t}\right)$ are the agent's private valuations for long and short positions:

$$
\begin{aligned}
& \hat{v}_{i t}^{+}\left(\delta \mid s^{t}\right)=\sum_{s} \frac{\hat{\lambda}_{i t+1}\left(s^{t}, s\right)+\left(1-\theta_{i}(\delta)\right) \hat{\mu}_{i t+1}\left(s^{t}, s\right)}{\hat{\lambda}_{i t}\left(s^{t}\right)}\left[q_{t+1}\left(s^{t}, s\right) \delta_{t+1}\left(s^{t}, s\right)+p_{t+1}\left(\delta \mid s^{t}, s\right)\right] \\
& \hat{v}_{i t}^{-}\left(\delta \mid s^{t}\right)=\sum_{s} \frac{\hat{\lambda}_{i t+1}\left(s^{t}, s\right)+\hat{\mu}_{i t+1}\left(s^{t}, s\right)}{\hat{\lambda}_{i t}\left(s^{t}\right)}\left[q_{t+1}\left(s^{t}, s\right) \delta_{t+1}\left(s^{t}, s\right)+p_{t+1}\left(\delta \mid s^{t}, s\right)\right]
\end{aligned}
$$

together with the complementary slackness condition for the incentive constraint:

$$
\begin{gathered}
\hat{\mu}_{i t+1}\left(s^{t}, s\right)\left\{\int\left(1-\theta_{i}(\delta)\right)\left[q_{t+1}\left(s^{t}, s\right) \delta_{t+1}\left(s^{t}, s\right)+p_{t+1}\left(\delta \mid s^{t}, s\right)\right] d N_{i t}^{+}\left(\delta \mid s^{t}\right)+\left(1-\theta_{i}(s)\right) a_{i t+1}^{+}\left(s^{t}, s\right)\right. \\
\left.-\int\left[q_{t+1}\left(s^{t}, s\right) \delta_{t+1}\left(s^{t}, s\right)+p_{t+1}\left(\delta \mid s^{t}, s\right)\right] d N_{i t}^{-}\left(\delta \mid s^{t}\right)-a_{i t+1}^{-}\left(s^{t}, s\right)\right\}=0 .
\end{gathered}
$$

Now, start from Theorem 1 and consider an equilibrium of the economy in which trees cannot be sold short, 
$(p, q, c, N)$. Let $a$ denote the implied Arrow security positions of the agents. Let $N^{+} \equiv N^{-} \equiv 0, a^{+} \equiv \max \{a, 0\}$ and $a^{-} \equiv \max \{-a, 0\}$. We seek to show that $\left(p, q, c, N^{+}, N^{-}, a^{+}, a^{-}\right)$is an equilibrium of the economy in which short-selling of tree is allowed, subject to the incentive constraint (15). Since the market-clearing conditions hold by construction, it is sufficient to show that $\left(c_{i}, N_{i}^{+}, N_{i}^{-}, a_{i}^{+}, a_{i}^{-}\right)$satisfies the first-order sufficient conditions (63)-(71), given the multipliers found in the first-order necessary conditions (37)-(40) in Proposition A.3.

- The first-order condtition (63) holds because it is identical to (37).

- Notice that, because the incentive constraint is not binding when $a_{i t+1}\left(s^{t}, s\right)>0$, we have that $\hat{\mu}_{i t+1}\left(s^{t}, s\right)=0$ whenever $a_{i t+1}\left(s^{t}, s\right)>0$. Hence, the first-order condition with respect to $W_{i t+1}\left(s^{t}, s\right)$, (38), implies the first-order conditions (64) and (65).

- The first-order condition (66) holds because it is identical to (65), given that (38) implies that $\hat{\lambda}_{i t}\left(s^{t}\right)=$ $\hat{\lambda}_{i t+1}\left(s^{t}, s\right)+\hat{\mu}_{t+1}\left(s^{t}, s\right)$.

- Next recall that $\hat{\lambda}_{i t}\left(s^{t}\right)=\hat{\lambda}_{i t+1}\left(s^{t}, s\right)+\hat{\mu}_{t+1}\left(s^{t}, s\right)$, so

$$
v_{i t}^{-}\left(\delta, \mid s^{t}\right)=\sum_{s}\left[q_{t+1}\left(s^{t}, s\right) \delta_{t+1}\left(s^{t}, s\right)+p_{t+1}\left(\delta \mid s^{t}, s\right)\right]
$$

But we know that trees are priced below replicating portfolios of Arrow securities, so $v_{i t}^{-}\left(\delta \mid s^{t}\right) \geq p_{t}\left(\delta \mid s^{t}\right)$, implying the first-order condition (67).

- Finally, complementary slackness condition holds by construction.

\section{Proof of Lemma D.1}

For sufficiency, start from a complete market equilibrium $(q, c)$ and define the tree pricing functional by the recursion:

$$
p_{t}\left(\delta \mid s^{t}\right)=\sum_{s} q_{t+1}\left(s^{t}, s\right)\left[\delta_{t+1}\left(s^{t}, s\right)+p_{t+1}\left(\delta \mid s^{t}, s\right)\right]
$$

for all nodes $\left(t, s^{t}\right), t<T$, with the convention that $p_{T}\left(s^{T}\right)=0$. Define as well the cash-on-hand of agent $i$ at node $\left(t, s^{t}\right)$ :

$$
q_{t}\left(s^{t}\right)\left(W_{i t}\left(s^{t}\right)-e_{i t}\left(s^{t}\right)\right)=\sum_{\left(u, s^{u}\right) \succeq\left(t, s^{t}\right)} q_{u}\left(s^{u}\right)\left(c_{i u}\left(s^{u}\right)-e_{i u}\left(s^{u}\right)\right) .
$$

Let $N$ denote the tree allocation in equation (47). We now verify that $(p, q, c, N)$ is an equilibrium when agents are subject to incentive constraints. First, by construction, all markets clear. Second, $U_{i}\left(c_{i}\right)$ is an upper bound for the agent maximum attainable utility, when she is subject to incentive constraints in addition to budget constraint. But, together with (73), (47) ensures that the plan $\left(c_{i}, N_{i}\right)$ is budget feasible and incentive compatible, so that this upper bound is attained. Hence, $\left(c_{i}, N_{i}\right)$ is optimal for each agent given the price system $(p, q)$.

Next, we turn to the proof of necessity. Suppose that $(q, c)$ is IC implementable. That is, there exists an equilibrium $(\hat{p}, \hat{q}, \hat{c}, \hat{N})$ with incentive constraints such that $\hat{q}=q$ and $\hat{c}=c$. Then, comparing the first-order condition in the complete market equilibrium and in the corresponding equilibrium with incentive constraints shows that the multiplier on the incentive constraint is equal to zero, and as a result the no-arbitrage condition (72) holds. This implies that cash-on-hand can be written as (73), and that (47) holds with $N=\hat{N}$. 


\section{Proof of properties of the zero-collateral equilibrium}

In this appendix we study properties of the zero-collateral equilibrium, that is an equilibrium in the special case when $\bar{N}(\Delta)=0$. First, we establish existence and uniqueness. Second, we establish continuity: equilibria can be made arbitrarily close to the zero collateral equilibrium, as long as $\bar{N}$ is small enough. This property is very convenient: it means that the zero-collateral equilibrium, which is straightforward to characterize, approximates all equilibria when $\bar{N}(\Delta) \simeq 0$.

Existence and uniqueness. Existence is straightforward but is not covered by our main Theorem, because an economy with zero collateral does not satisfy our assumption (1) that aggregate dividend are strictly positive at all nodes. Uniqueness is specific to this case and implies a continuity property: equilibria converge to the unique zero-collateral equilibrium as $\bar{N}(\Delta) \rightarrow 0$.

Proposition VIII.1 Suppose that $e \gg 0$ and $\bar{N}=0$. Then, in equilibrium, agents consume their labor endowment, $c_{i t}\left(s^{t}\right)=e_{i t}\left(s^{t}\right)$ and $N_{i}\left(s^{t}\right)=0$ for all agents $i$ and nodes $\left(t, s^{t}\right)$. Moreover, an equilibrium price system is given by $q_{0}\left(s^{0}\right)=1$,

$$
\begin{aligned}
\frac{q_{t+1}\left(s^{t}, s\right)}{q_{t}\left(s^{t}\right)} & =\max _{i} \beta \pi_{t+1}\left(s \mid s^{t}\right) \frac{u_{i}^{\prime}\left(e_{i t+1}\left(s^{t}, s\right)\right)}{u_{i}^{\prime}\left(e_{i t}\left(s^{t}\right)\right)} \\
p_{t}\left(\delta \mid s^{t}\right) & =\max _{i} \sum_{s}\left(1-\theta \frac{\hat{\mu}_{i t+1}\left(s^{t}, s\right)}{\hat{\lambda}_{i t}\left(s^{t}\right)}\right)\left[q_{t+1}\left(s^{t}, s\right) \delta_{t+1}\left(s^{t}, s\right)+p_{t+1}\left(\delta \mid s^{t}, s\right)\right]
\end{aligned}
$$

where $\hat{\lambda}_{i t}\left(s^{t}\right)=\beta^{t} \pi_{t}\left(s^{t}\right) u_{i}^{\prime}\left(e_{i t}\left(s^{t}\right)\right) / q_{t}\left(s^{t}\right)$, and $\hat{\mu}_{i t+1}\left(s^{t}, s\right)=\hat{\lambda}_{i t}\left(s^{t}\right)-\hat{\lambda}_{i t+1}\left(s^{t}, s\right)$.

We first show that the proposed price system and allocation is an equilibrium. Indeed, it is obvious that the proposed allocation clears market and that, given the proposed price system, it satisfies the budget and incentive constraints of each agent. Optimality follows from the first-order conditions of Proposition A.3.

Next, we turn to uniqueness of the equilibrium allocation. Consider any equilibrium $(p, q, c, N)$. Then, since $\bar{N}=$ 0 , we must have that $N_{i}=0$ as well. The incentive constraints for agent $i$ then implies that $W_{i t}\left(s^{t}\right) \geq e_{i t}\left(s^{t}\right)$ for all $t \geq$ 1. But notice that, in equilibrium, at all nodes, $\sum_{i} q_{t}\left(s^{t}\right) W_{i t}\left(s^{t}\right)=\sum_{i} q_{t}\left(s^{t}\right) e_{i t}\left(s^{t}\right)+\int\left[q_{t}\left(s^{t}\right) \delta_{t}\left(s^{t}\right)+p_{t}\left(\delta \mid s^{t}\right)\right] d \bar{N}(\delta)$. ${ }^{25}$ This implies that $W_{i t}\left(s^{t}\right)=e_{i t}\left(s^{t}\right)$ at all $t \geq 1$. The definition of time-zero cash-on-hand also implies that $W_{i 0}\left(s^{0}\right)=e_{i 0}\left(s^{0}\right)$ at $t=0$. The sequential budget constraint then implies that $c_{i t}\left(s^{t}\right)=e_{i t}\left(s^{t}\right)$.

Continuity. Now we argue that, the zero-collateral equilibrium identified above approximates all equilibria as $\bar{N} \rightarrow 0$.

Proposition VIII.2 Fix some endowment of labor income $e \gg 0$ and some sequence of tree supplies $\bar{N}^{\ell}$ such that $\bar{N}^{\ell}(\Delta) \rightarrow 0$, satisfying our maintained assumption (1) that aggregate dividend is strictly positive at all nodes. Consider any associated sequence of equilibria $\left(p^{\ell}, q^{\ell}, c^{\ell}, N^{\ell}\right)$, and the corresponding sequence of optimal payoff sets, $X^{\ell}$, and assume that the tree pricing equation (25) holds for all trees in $\Delta$. Let $(p, q, c, N)$ denote the zero-collateral equilibrium identified in Proposition VIII.1, and $X$ the corresponding optimal payoff sets. Then $\left(p^{\ell}, q^{\ell}, c^{\ell}, N^{\ell}\right) \rightarrow(p, q, c, N)$. Moreover, for any sequence of payoffs $x^{\ell} \in X_{j t}^{\ell}\left(s^{t}\right)$, if $x^{\ell} \rightarrow x$, then $x \in X_{j t}\left(s^{t}\right)$.

${ }^{25}$ In the last period, $t=T$, this is simply a restatement of market clearing since $W_{T}=c_{T}$ and $p_{T}=0$. Suppose that this result is true at $t+1 \leq T$. Then, if we add all the sequential budget constraints (31) across all agents and use our induction hypothesis, we obtain:

$$
\sum_{i} q_{t}\left(s^{t}\right) c_{i t}\left(s^{t}\right)+\sum_{i} \int p_{t}\left(\delta \mid s^{t}\right) d N_{i t}\left(\delta \mid s^{t}\right)=\sum_{i} q_{t}\left(s^{t}\right) W_{i t}\left(s^{t}\right),
$$

and the result follows from the market clearing condition for consumption and trees at node $\left(t, s^{t}\right)$. 
Let us first extract a subsequence converging to some $(\hat{p}, \hat{q}, \hat{c}, \hat{N})$. Since $\bar{N}^{\ell} \rightarrow 0$, it follows that $N_{i}^{\ell} \rightarrow 0$ for all $i$ and, therefore, $\hat{N}=0$. Next, after passing to the limits in budget and incentive constraints, and noting that $\hat{q}>0,{ }^{26}$ we can use the same argument as in the proof of uniqueness in Proposition VIII.1 to obtain that $\hat{c}=c=e$. Finally, given that $e_{i t}\left(s^{t}\right)>0$, we can pass to the limit in the first-order conditions and obtain that $\hat{p}=p$ and $\hat{q}=q$.

For the second result, let $Y_{i t}\left(s^{t}\right)$ denote the intersection of the optimal payoff set of agent $i$ at node $\left(t, s^{t}\right)$ with the simplex. It solves the optimization problem:

$$
Y_{i t}\left(s^{t}\right)=\arg \max \left\{Q_{i t+1}\left(s^{t}\right) \cdot x-P_{t}\left(x \mid s^{t}\right)\right\}
$$

with respect to $x \in \mathbb{R}_{+}^{S}$ such that $\sum x(s)=1$ and where, with some abuse of notation $P\left(x \mid s^{t}\right) \equiv \max _{j} Q_{j t+1} \cdot x$ is the price of payoff $x$. Since the objective of this optimization problem is continuous in the private valuations $Q_{j}$, Berge Theorem implies that its arg max is upper hemi continuous in the private valuations. Now consider any sequence $x^{\ell} \in X_{i t}^{\ell}\left(s^{t}\right)$ such that $x^{\ell} \rightarrow x$. If $x=0$, then the result follows because 0 belongs to all payoff sets. If $x \neq 0$, then the sequence $y^{\ell}=x^{\ell} / \sum x^{\ell}(s)$ belongs to the intersection of the optimal payoff set with the simplex, $Y_{i t}^{\ell}\left(s^{t}\right)$, which are upper-hemi continuous in private valuations. Moreover, by the continuity result we just established above, $Q_{i}^{\ell} \rightarrow Q_{i}$ for all $i$. Therefore, we obtain that $x / \sum x(s)$ belongs to the limit intersection, $Y_{i t}\left(s^{t}\right)$ and, by implication, that $x$ belongs to the limit optimal payoff set, $X_{i t}\left(s^{t}\right)$.

\section{Optimal payoff sets and power-diagrams}

Power diagrams: a definition. Take some finite collection of $S$-dimensional vectors $a=\left(a_{1}, \ldots, a_{I}\right)$ and scalars $b=\left(b_{1}, \ldots, b_{I}\right)$. Then, the power diagram generated by $(a, b)$ is the collection of sets:

$$
Y_{i} \equiv\left\{x \in \mathbb{R}^{S}: a_{i} \cdot x+b_{i} \geq a_{j} \cdot x+b_{j} \text { for all } j\right\}
$$

It is clear from the definition that any $x \in \mathbb{R}^{S}$ must belong to some $Y_{i}$, that is, a power diagram covers $\mathbb{R}^{S}$. Aurenhammer $(1987 \mathrm{a}, \mathrm{b})$ has shown that any covering generated by a power diagram can be viewed as the projections of a $S+1$ dimensional convex polyhedron on $\mathbb{R}^{S}$, and vice versa. Put differently, think of lying on the floor (that would be $\mathbb{R}^{S}$ ) and taking a picture of a $S+1$-dimensional polyhedron from your position: you would produce a $S$-dimensional picture that is a covering of $\mathbb{R}^{S}$. Moreover, it is easy to see that this picture is generated by a power diagram and vice versa. Indeed, consider the polyhedron defined as:

$$
\left\{(x, y) \in \mathbb{R}^{S} \times \mathbb{R}: y \geq \max _{j}\left\{a_{j} \cdot x+b_{j}\right\}\right\} .
$$

Then it is clear that $Y_{i}$ can be viewed as the face of the polyhedron defined by $y=a_{i} \cdot x+b_{i}$ and $y \geq a_{j} \cdot x+b_{j}$ for all $j$.

Any collection of optimal payoff sets map into some $S-1$ dimensional power diagram. Since optimal payoff sets are cones, they are entirerly determined by their intersection with the $S-1$ dimensional simplex. Moreover, consider any $x$ in the $S-1$-dimensional simplex, that is such that $x(s) \geq 0$ and $\sum_{s=1}^{S} x(s)=1$. Then, at

\footnotetext{
${ }^{26}$ This follows by an argument in our existence proof: if $\hat{q}_{t}\left(s^{t}\right)=0$ for some $\left(t, s^{t}\right)$, consumptions would become unbounded as $\ell \rightarrow \infty$, contradicting market clearing.
} 
any node $\left(t, s^{t}\right), x \in X_{i t}\left(s^{t}\right)$ if and only if

$$
\begin{aligned}
& \sum_{s=1}^{S} Q_{i t+1}\left(s^{t}, s\right) x(s) \geq \sum_{s=1}^{S} Q_{j t+1}\left(s^{t}, s\right) x(s) \text { for all } j \\
\Leftrightarrow & \sum_{s=1}^{S-1} a_{i t+1}\left(s^{t}, s\right) x(s)+b_{i t}\left(s^{t}\right) \geq \sum_{s=1}^{S-1} a_{i t+1}\left(s^{t}, s\right) x(s)+b_{i t}\left(s^{t}\right) \text { for all } j,
\end{aligned}
$$

where $a_{i t+1}\left(s^{t}, s\right) \equiv Q_{i t+1}\left(s^{t}, s\right)-Q_{i t+1}\left(s^{t}, S\right)$ and $b_{i t}\left(s^{t}\right) \equiv Q_{i t+1}\left(s^{t}, S\right)$. Therefore, the optimal payoff sets can be viewed as the intersection of a $S$-1-dimensional power-diagrams with the $S$-1-dimensional simplex.

Any $S-1$ dimensional power diagrams maps into a collection of optimal payoff sets. The converse is also true. Given any $S-1$ dimensional power diagram, then there exists an economy such that the collection of optimal payoff sets correspond to the intersection of this power-diagram with the $S-1$ simplex. Namely, consider, at each node $\left(t, s^{t}\right), t<T$, a $S-1$ power diagram described by coefficients $\left(a_{t+1}\left(s^{t}\right), b_{t+1}\left(s^{t}\right)\right)$ as above. Let

$$
\begin{aligned}
\Psi_{i t+1}\left(s^{t}, s\right) & \equiv a_{i t+1}\left(s^{t}, s\right)+b_{i t}\left(s^{t}\right)+K, \text { for } s \in\{1,2, \ldots, S-1\} \\
\Psi_{i t+1}\left(s^{t}, S\right) & \equiv b_{i t}\left(s^{t}\right)+K
\end{aligned}
$$

where $K$ is some constant that is large enough so that all $\Psi_{i t+1}\left(s^{t}, s\right)>0$ for all agents and all nodes. Then consider an economy with $I$ agents, all endowed with identical CRRA preferences with parameter $\gamma$. Define the endowment of agent $i$ recursively as

$$
e_{i t+1}\left(s^{t}, s\right)=g_{i t+1}\left(s^{t}, s\right) e_{i t}\left(s^{t}, s\right) \text {, }
$$

with initial condition $e_{i 0}\left(s^{0}\right)=1$, and where the growth rate is taken to solve:

$$
\beta \pi_{t+1}\left(s \mid s^{t}\right) g_{i t+1}\left(s^{t}, s\right)^{-\gamma}=\Psi_{i t+1}\left(s^{t}, s\right) .
$$

If there is no collateral, $\bar{N}=0$, it follows from the results in Section VIII that the private valuations $Q_{i t+1}\left(s^{t}, s\right)$ for Arrow-Debreu securities take the form:

$$
Q_{i t+1}\left(s^{t}, s\right)=(1-\theta) \max _{j} \Psi_{j t+1}\left(s^{t}, s\right)+\theta \Psi_{i t+1}\left(s^{t}, s\right)
$$

But the first vector of the sum, $(1-\theta) \max _{j} \Psi_{j t+1}\left(s^{t}, s\right)$, is independent of $i$. It follows that $Q_{i t+1}\left(s^{t}\right) \cdot x \geq Q_{j t+1}\left(s^{t}\right) \cdot x$ for all $j$ if and only if $\Psi_{i t+1}\left(s^{t}, s\right) \cdot x \geq \Psi_{j t+1}\left(s^{t}, s\right) \cdot x$ for all $j$, and so if and only if the intersection of the optimal payoff sets with the $S-1$ dimensional simplex belongs to power diagram we started with.

Assumptions and computations for Figure 3. Focus on two time periods $t$ and $t+1$ in an economy with $I=50$ agents with identical relative risk aversion $\gamma=1$ and discount factor $\beta=0.95$. There are three states, one disaster state $s=1$ and two normal states, $s=2$ and $s=3$. State $s=1$ is a disaster state with dismal endowment growth $g(3)=0.8$, and realizes with low probability $\pi(3)=0.05$. State $s=2$ is a normal state with low endowment growth $g(2)=1.01$, and realizes with probability $\pi(2)=0.475$. State $s=3$ is a normal state with high endowment growth $g(3)=1.03$, and realizes with probability $\pi(3)=0.475$. We assume that all agents have the same labor 
income at time $t$, normalized to $e_{i t}\left(s^{t}\right)=1$. At time $t+1$, on the other hand:

$$
\frac{e_{i t+1}\left(s^{t}, s\right)}{e_{i t}\left(s^{t}\right)}=\alpha_{i}(s) g(s),
$$

where the $\alpha_{i}(s)$ are drawn at random and are subsequently normalized so that $g(s)$ represents the aggregate growth of income in state $s$. We assume that there is no collateral, $\bar{N}(\Delta)=0$, so that the intertemporal marginal rate of substitution of agent $i$ is given by $\beta \pi_{t+1}\left(s \mid s^{t}\right)\left(\alpha_{i}(s) g(s)\right)^{-\gamma}$. In Appendix VIII we show that equilibria are continuous in $\bar{N}$ near $\bar{N}(\Delta)=0$ : hence, the optimal payoff sets with $\bar{N}=0$ approximate those with $\bar{N} \simeq 0$.

Next, we reduce the dimension of the problem by studying the intersection of $X_{i}$ with the simplex, as outlined above. Namely, proceeding as above, direct calculations reveals that this intersection is made up of points $x \in \mathbb{R}_{+}^{2}$, $x(1)+x(2) \leq 1$, such that

$$
a_{i} \cdot x+b_{i} \geq a_{j} \cdot x+b_{j} \text { for all } j
$$

where

$$
\begin{aligned}
\Psi_{i}(s) & =\beta \pi(s)\left[\alpha_{i}(s) g(s)\right]^{-\gamma} \\
a_{i}(1) & =\Psi_{i}(1)-\Psi_{i}(3) \\
a_{i}(2) & =\Psi_{i}(2)-\Psi_{i}(3) \\
b_{i} & =\Psi_{i}(3) .
\end{aligned}
$$

To calculate the optimal payoff sets, we use the transport package in $\mathrm{R}$, which requires to reformulate the inequalities $a_{i} \cdot y+b_{i} \geq a_{j} \cdot y+b_{j}$ as

$$
\left\|y-z_{i}\right\|^{2}-w_{i} \leq\left\|y-z_{j}\right\|^{2}-w_{j}
$$

for some $z_{i} \in \mathbb{R}^{2}$ and $w_{i} \geq 0$. Direct calculations show that $z_{i}=a_{i} / 2$ and $w_{i}=\left\|z_{i}\right\|^{2}+b_{i}$.

The calculation. Figure 3 is created using R, in the following steps. First, we use the fonction power_diagram, from the transport pacakge, to calculate the optimal payoff sets. Precisely, this function calculates the vertices of the polygons that enclose the intersection of optimal payoff sets with the unit square, $[0,1] \times[0,1]$. We then calculate the intersection of these polygons with the simplex, using the function st_intersection from the sf package. Finally, we plot these polygons in barycentric coordinates using the function geom_polygon of the ggplot2 package.

\section{$\mathrm{X}$ Parametric cases not covered in Proposition 3}

Case $\gamma \phi=1$. Then $V(\alpha, x)$ is linear in $\alpha$ and one can directly verify that the optimal payoff set of $\alpha_{1}=0$ is $X_{0}=\left[0, x^{\star}(0)\right]$, where $x^{\star}(0)$ is defined in equation (51). The optimal payoff set for $\alpha_{I}$ is $X_{I}=\left[x^{\star}(0), 1\right]$. For all other agents $X_{i}=\left\{x^{\star}(0)\right\}$.

Case $\gamma \phi>1$. In this case the function $\alpha \mapsto V(\alpha, x)$ is strictly convex in $\alpha$. This implies that it achieves a strict maximum either at $\alpha_{1}$ or at $\alpha_{I}$. Therefore there exists some $x^{\star}$ such that $X_{1}=\left[0, x^{\star}\right]$ and $X_{I}=\left[x^{\star}, 1\right]$. This 
threshold solves $V\left(\alpha_{1}, x^{\star}\right)=V\left(\alpha_{I}, x^{\star}\right)$, which leads to:

$$
x^{\star}=\frac{\pi_{2}\left(1-\left(1-k_{2} \alpha_{I}\right)^{\phi \gamma}\right)}{\pi_{1}\left(\left(1+k_{1} \alpha_{I}\right)^{\phi \gamma}-1\right)+\pi_{2}\left(1-\left(1-k_{2} \alpha_{I}\right)^{\phi \gamma}\right)} .
$$

For all other $i, X_{i}=\emptyset$.

\section{X.1 Proof that the pricing functional solves (30)}

The proof follows a standard-optimality verification argument. Let $\hat{P}_{t}\left(\delta s^{t}\right)$ denotes the solution of the Bellman equation (25) for tree $\delta$, and let $\hat{J}$ denote a sequence of agent that solve the Bellman equation for tree $\delta$ at each node. Now take any stream of asset holders $J$. The Bellman equation (30) and the definition of $q_{t}\left(s^{t} \mid J\right)$ imply that at node $\left(u, s^{u}\right), u<T$ :

$$
\hat{P}_{u}\left(\delta \mid s^{u}\right) \geq \sum_{s} \frac{q_{u+1}\left(s^{u} \mid J\right)}{q_{u}\left(s^{u} \mid J\right)}\left[\delta_{u+1}\left(s^{u}, s\right)+\hat{P}_{u+1}\left(\delta \mid s^{u}, s\right)\right],
$$

with an equality if $j$ is the term $\left(t, s^{t}\right)$ of the sequence of agent $\hat{J}$ solving the Bellman equation. Multiplying through by $q_{u}\left(s^{u} \mid J\right)$, dividing by $q_{t}\left(s^{t} \mid J\right)$ and adding the inequalities for all $s^{u} \succeq s^{t}$, we obtain that

$$
\hat{P}_{t}\left(\delta \mid s^{t}\right) \geq \sum_{\left(u, s^{u}\right) \succ\left(t, s^{t}\right)} \frac{q_{u}\left(s^{u} \mid J\right)}{q_{t}\left(s^{t} \mid J\right)} \delta_{u}\left(s^{u}, s\right)
$$

with an equality if $J=\hat{J}$. Therefore $\hat{P}\left(\delta \mid s^{t}\right)$ is an upper bound for $P\left(\delta \mid s^{t}\right)$, and it is attained for $\hat{J}$. The result follows.

\section{An micro-foundation à la Rampini and Viswanathan (2010)}

In this section we offer an alternative micro-foundation of the incentive constraint (6). We closely follow the approach Rampini and Viswanathan (2010) and adapt their argument to our setting.

Precisely, let us imagine a contracting problem between an agent of our model and a continuum of competitive lenders. Agents and lenders take as given some no-arbitrage security prices $(p, q) \in \mathbb{N} A$, i.e. the prices of trees $p$ and of Arrow securities $q$. As everywhere else in this Appendix, $(p, q)$ denote time-zero deflated prices. Lenders can commit to make state-contingent payments to the agent, but they cannot hold trees: they cannot operate the technology that produces the corresponding dividend streams. The agent, on the other hand, can operate the technology but she has limited commitment. At any node $\left(t, s^{t}\right)$, the agent can default, divert a fraction $\theta$ of the cum-dividend value of her tree portfolio, contract with a new competitive lender and continue to receive her stream of labor income. Agents maximize the same intertemporal utility as in the paper, while lenders maximizes the present value of their profits, evaluated at Arrow security prices.

\section{XI.1 The optimal contracting problem}

A contract is a list $(c, N, \tau)$ that specifies, for all nodes $\left(t, s^{t}\right)$, the consumption and tree holdings of the agent, $c_{t}\left(s^{t}\right)$ and $N_{t}\left(s^{t}\right)$, as well as the net transfers made by the lender to the agent, $\tau_{t}\left(s^{t}\right)$. We omit the type " $i$ " subscript for notational simplicity. A contract is feasible if it satisfies the budget, participation, and enforcement constraints specified below. 
The key difficulty is to formulate the enforcement constraint at node $\left(t, s^{t}\right)$, because it depends on the value of defaulting multiple times with successive lenders. Specifically, the enforcement constraint is determined by the value of defaulting and re-contracting with a new lender, which itself depends on the option to defaulting and re-contracting later with yet another lender, and so on. This implies that the enforcement constraint at node $\left(t, s^{t}\right)$ depends on the enforcement constraint at successor nodes, $\left(u, s^{u}\right) \succ\left(t, s^{t}\right)$. Hence, it is natural to define the enforcement constraint recursively, by backward induction.

Namely, we first define the set of feasible contract at a terminal node, $B_{T}\left(w \mid s^{T}\right)$, where $w$ is the agent's wealth, to be the collection of contracts $(c, N, \tau)$ such that

$$
\begin{aligned}
& q_{T}\left(s^{T}\right) c_{T}\left(s^{T}\right)=q_{T}\left(s^{T}\right) w+q_{T}\left(s^{T}\right) e_{T}\left(s^{T}\right)+q_{T}\left(s^{T}\right) \tau_{T}\left(s^{T}\right) \\
& q_{T}\left(s^{T}\right) \tau_{T}\left(s^{T}\right)=0
\end{aligned}
$$

i.e., the contracts that satisfy the budget constraint and the binding participation constraint of the lender, which we state as an equality for simplicity. Note that, at the terminal node, there are no trees left to purchase. As noted above, all valuations are based on time zero prices $(p, q)$. We do not include an enforcement constraints at time $T$ in $B_{T}\left(w \mid s^{T}\right)$ : instead, we include these enforcement constraints at $T-1$, as explained below.

Now, proceeding by backward induction, consider any node $\left(t, s^{t}\right)$, with $t<T$ and suppose that we have already constructed sets of feasible contracts, $B_{u}\left(w \mid s^{u}\right)$, for all successor nodes $\left(u, s^{u}\right) \succ\left(t, s^{t}\right)$. At node $\left(t, s^{t}\right)$, we define $B_{t}\left(w \mid s^{t}\right)$ to be the set of contracts $(c, N, \tau)$ satisfying the following constraints. First, a budget constraint at $\left(t, s^{t}\right)$ :

$$
q_{t}\left(s^{t}\right) c_{t}\left(s^{t}\right)+\int p_{t}\left(\delta \mid s^{t}\right) d N_{t}\left(\delta s^{t}\right)=q_{t}\left(s^{t}\right) w+q_{t}\left(s^{t}\right) e_{t}\left(s^{t}\right)+q_{t}\left(s^{t}\right) \tau_{t}\left(s^{t}\right)
$$

Second a budget constraint at all $\left(u, s^{u}\right) \succ\left(t, s^{t}\right)$,

$$
\begin{aligned}
& q_{u}\left(s^{u}\right) c_{u}\left(s^{u}\right)+\int p_{u}\left(\delta \mid s^{u}\right) d N_{u}\left(\delta s^{u}\right) \\
= & \int\left[q_{u}\left(s^{u}\right) \delta_{u}\left(s^{u}\right)+p_{u}\left(\delta \mid s^{u}\right)\right] d N_{u-1}\left(\delta \mid s^{u-1}\right)+q_{u}\left(s^{u}\right) e_{u}\left(s^{u}\right)+q_{u}\left(s^{u}\right) \tau_{u}\left(s^{u}\right) .
\end{aligned}
$$

Third, the binding participation constraint for the lender at $\left(t, s^{t}\right)$ :

$$
\sum_{\left(u, s^{u}\right) \succeq\left(t, s^{t}\right)} q_{u}\left(s^{u}\right) \tau_{u}\left(s^{u}\right)=0 .
$$

Fourth, a set of enforcement constraints for the agent, that ensures that, at all successor nodes $\left(u, s^{u}\right) \succ\left(t, s^{t}\right)$, the agent does not have incentive to default:

$$
\sum_{\left(v, s^{v}\right) \succ\left(u, s^{u}\right)} \beta^{v} \pi_{v}\left(s^{v}\right) u\left(c_{v}\left(s^{v}\right)\right) \geq \sum_{\left(v, s^{v}\right) \succ\left(u, s^{u}\right)} \beta^{v} \pi_{v}\left(s^{v}\right) u\left(\hat{c}_{v}\left(s^{v}\right)\right)
$$

for all $(\hat{c}, \hat{N}, \hat{\tau}) \in B_{u}\left(\hat{w}_{u}\left(s^{u}\right) \mid s^{u}\right)$, where

$$
q_{u}\left(s^{u}\right) \hat{w}_{u}\left(s^{u}\right)=\theta \int\left[q_{u}\left(s^{u}\right) \delta_{u}\left(s^{u}\right)+p_{u}\left(\delta \mid s^{u}\right)\right] d N_{u-1}\left(\delta \mid s^{u-1}\right)
$$


is the value of the agent's tree portfolio after default and diversion. This last constraint states formally that the agent can default, divert a fraction $\theta$ of the cum-dividend value of its portfolio and pick a feasible contract with a new lender. This new contract gives the agent the option to default at some future date. In particular, as anticipated above, the enforcement constraints at node $\left(t, s^{t}\right)$ depends on future enforcement constraints encoded in the sets $B_{u}\left(\hat{w}_{u}\left(s^{u}\right) \mid s^{u}\right)$.

The optimal contracting problem is to choose $(c, N, \tau) \in B_{0}\left(w_{0} \mid s^{0}\right)$, where $w_{0}$ is the cum-dividend value of the agent's initial tree portfolio endowment.

\section{XI.2 Implementation with Arrow borrowing and incentive constraints}

The main result is that the agent's problem considered in the text, with incentive constraints of the form (6), yields the same allocation as the optimal contracting problem defined above. Formally:

Proposition XI.1 Consider a solution $(c, N, a)$ to the agent's problem considered in the text, and let

$$
q_{t}\left(s^{t}\right) \tau_{t}\left(s^{t}\right) \equiv q_{t}\left(s^{t}\right) a_{t}\left(s^{t}\right)-\sum_{s} q_{t+1}\left(s^{t}, s\right) a_{t+1}\left(s^{t}, s\right) .
$$

Then $(c, N, \tau)$ solves the optimal contracting problem.

The proof proceeds in two steps.

Step 1 of the proof. Similarly to Rampini and Viswanathan (2010) we show that the enforcement constraint implies:

Lemma XI.1 If $(c, N, \tau) \in B_{0}\left(w_{0} \mid s^{0}\right)$ then, at all nodes $\left(t, s^{t}\right), t>0$ :

$$
-\sum_{\left(u, s^{u}\right) \succeq\left(t, s^{t}\right)} q_{u}\left(s^{u}\right) \tau_{u}\left(s^{u}\right) \leq(1-\theta) \int\left[q_{t}\left(s^{t}\right) \delta_{t}\left(s^{t}\right)+p_{t}\left(\delta \mid s^{t}\right)\right] d N_{t-1}\left(\delta \mid s^{t-1}\right) .
$$

According to (78), the present value of net transfers to the lender cannot must be less than $1-\theta$ times the cumdividend value of the tree portfolio acquired in the previous period. The proof, which is identical to that in Rampini and Viswanathan (2010), proceeds by contradiction. Suppose that (78) does not hold at some node $\left(t, s^{t}\right)$. Then, we argue that the incentive constraint (77) is violated at this node. Indeed, the agent could default, start over with $\hat{w}_{t}\left(s^{t}\right)=\theta \int\left[q_{t}\left(s^{t}\right) \delta_{t}\left(s^{t}\right)+p_{t}\left(\delta \mid s^{t}\right)\right] d N_{t-1}\left(\delta \mid s^{t-1}\right)$ at node $\left(t, s^{t}\right)$ and pick a new contract $(\hat{c}, \hat{N}, \hat{\tau}) \in B_{t}\left(\hat{w}_{t}\left(s^{t}\right) \mid s^{t}\right)$ that changes the transfer and consumption at node $\left(t, s^{t}\right)$, but keeps everything else the same. Namely, the transfer at $\left(t, s^{t}\right)$ is changed to:

$$
q_{t}\left(s^{t}\right) \hat{\tau}\left(s^{t}\right)=-\sum_{\left(u, s^{u}\right) \succ\left(t, s^{t}\right)} q_{t}\left(s^{t}\right) \tau_{t}\left(s^{t}\right)
$$

while consumption is changed to:

$$
\begin{aligned}
& q_{t}\left(s^{t}\right)\left[\hat{c}_{t}\left(s^{t}\right)-c_{t}\left(s^{t}\right)\right] \\
= & -(1-\theta) \int\left[q_{t}\left(s^{t}\right) \delta_{t}\left(s^{t}\right)+p_{t}\left(\delta \mid s^{t}\right)\right] d N_{t-1}\left(\delta \mid s^{t-1}\right)-\sum_{\left(u, s^{u}\right) \succeq\left(t, s^{t}\right)} q_{u}\left(s^{u}\right) \tau_{u}\left(s^{u}\right)
\end{aligned}
$$


which is strictly positive by our maintained assumption. The budget constraints and the participation constraint of the lenders hold by construction. Moreover, since the original contract satisfies the enforcement constraint at all nodes $\left(u, s^{u}\right) \succ\left(t, s^{t}\right)$, so does the new contract. But this means that the enforcement constraint does not hold at $\left(t, s^{t}\right)$, a contradiction.

Step 2 of the proof. With the Lemma in mind, we define the relaxed problem to be the optimal contracting problem in which all enforcement constraints are replaced by (78). Clearly the Lemma shows that the constraint set in the original problem is contained in that of the relaxed problem. Hence, the value of the relaxed problem is an upper bound to the value of the original problem. Next, we show that this upper bound is attained:

Lemma XI.2 The value of the original contracting problem is equal to that of the relaxed contracting problem, and to the agent's problem considered in the text.

To establish this result we make the same change of variable as in Rampini and Viswanathan (2010), namely we let:

$$
\begin{aligned}
q_{t}\left(s^{t}\right) a_{t}\left(s^{t}\right) & \equiv \sum_{\left(u, s^{u}\right) \succeq\left(t, s^{t}\right)} q_{u}\left(s^{u}\right) \tau_{u}\left(s^{u}\right) \\
\Leftrightarrow \quad q_{t}\left(s^{t}\right) \tau_{t}\left(s^{t}\right) & =q_{t}\left(s^{t}\right) a_{t}\left(s^{t}\right)-\sum_{s} q_{t+1}\left(s^{t}, s\right) a_{t+1}\left(s^{t}, s\right),
\end{aligned}
$$

with $a_{0}\left(s^{0}\right)=0$. This leads to an equivalent representation of the constraint set of the relaxed problem. Namely, a contract $(c, N, a)$ belongs to the constraint set of the relaxed problem starting at time $t$ given initial financial wealth $w$ if it satisfies the following constraints. First, a budget constraint at $\left(t, s^{t}\right)$ :

$$
q_{t}\left(s^{t}\right) c_{t}\left(s^{t}\right)+\int p_{t}\left(\delta \mid s^{t}\right) d N_{t}\left(\delta s^{t}\right)+\sum_{s} q_{t+1}\left(s^{t}, s\right) a_{t+1}\left(s^{t}, s\right)=q_{t}\left(s^{t}\right) w+q_{t}\left(s^{t}\right) e_{t}\left(s^{t}\right)
$$

Second a budget constraint at all $\left(u, s^{u}\right) \succ\left(t, s^{t}\right)$,

$$
\begin{aligned}
& q_{u}\left(s^{u}\right) c_{u}\left(s^{u}\right)+\int p_{u}\left(\delta \mid s^{u}\right) d N_{u}\left(\delta \mid s^{u}\right)+\sum_{s} q_{u+1}\left(s^{u}, s\right) a_{u+1}\left(s^{u}, s\right) \\
= & \int\left[q_{u}\left(s^{u}\right) \delta_{u}\left(s^{u}\right)+p_{u}\left(\delta \mid s^{u}\right)\right] d N_{u-1}\left(\delta \mid s^{u-1}\right)+q_{u}\left(s^{u}\right) e_{u}\left(s^{u}\right)+q_{u}\left(s^{u}\right) a_{u}\left(s^{u}\right) .
\end{aligned}
$$

with the convention that $N_{T}=a_{T+1}=0$. Third a set of incentive constraints for for all $\left(u, s^{u}\right) \succ\left(t, s^{t}\right)$, which take the same form as (6):

$$
-q_{u}\left(s^{u}\right) a_{u}\left(s^{u}\right) \leq(1-\theta) \int\left[q_{u}\left(s^{u}\right) \delta_{u}\left(s^{u}\right)+p_{u}\left(\delta \mid s^{u}\right)\right] d N_{u-1}\left(\delta \mid s^{u-1}\right)
$$

Clearly the relaxed optimal contracting problem at time $t=0$ with wealth $w_{0}$ has become identical to the agent's problem studied in the text.

Now let $V_{t}\left(w \mid s^{t}\right)$ denote the value of the relaxed contracting problem starting with financial wealth $w$ at $\left(t, s^{t}\right)$. Clearly, this value function is weakly increasing in $w$. Moreover a standard dynamic programming argument shows that if $(c, N, a)$ solves the relaxed contracting problem starting at time zero given $w_{0}$, then it also solves it starting 
at node $\left(t, s^{t}\right)$ given financial wealth

$$
q_{t}\left(s^{t}\right) w_{t}\left(s^{t}\right)=\int\left[q_{t}\left(s^{t}\right) \delta_{t}\left(s^{t}\right)+p_{t}\left(\delta \mid s^{t}\right)\right] d N_{t-1}\left(\delta \mid s^{t-1}\right)+a_{t}\left(s^{t}\right)
$$

We now verify that a solution of the relaxed contracting problem is incentive compatible for the original contracting problem (after doing the opposite change of variables from $a$ to $\tau$ ). Indeed, the budget constraints and participation constraint hold by construction. The enforcement constraint (77) hold as well at all $\left(t, s^{t}\right), t>0$. Indeed, the left-hand side of the enforcement constraint is equal to $V_{t}\left(w_{t}\left(s^{t}\right) \mid s^{t}\right)$ defined above. The right-hand side is bounded above by the solution of the relaxed contracting problem, starting with financial wealth

$$
q_{t}\left(s^{t}\right) \hat{w}_{t}\left(s^{t}\right)=\theta \int\left[q_{t}\left(s^{t}\right) \delta_{t}\left(s^{t}\right)+p_{t}\left(\delta \mid s^{t}\right)\right] d N_{t-1}\left(\delta \mid s^{t-1}\right) .
$$

Since the incentive constraint (82) holds at $\left(t, s^{t}\right)$, we have that $w_{t}\left(s^{t}\right) \geq \hat{w}_{t}\left(s^{t}\right)$. Since the value function is weakly increasing, $V_{t}\left(w_{t}\left(s^{t}\right) \mid s^{t}\right) \geq V_{t}\left(\hat{w}_{t}\left(s^{t}\right) \mid s^{t}\right)$. Therefore, the enforcement constraint holds. 